\title{
THE SPECTRUM ASYMPTOTICS FOR THE DIRICHLET PROBLEM IN THE CASE OF THE BIHARMONIC OPERATOR IN A DOMAIN WITH HIGHLY INDENTED BOUNDARY
}

\author{
V. A. KOZLOV AND S. A. NAZAROV \\ Dedicated to Vasilǐ Mikhălovich Babich
}

\begin{abstract}
Asymptotic expansions are constructed for the eigenvalues of the Dirichlet problem for the biharmonic operator in a domain with highly indented and rapidly oscillating boundary (the Kirchhoff model of a thin plate). The asymptotic constructions depend heavily on the quantity $\gamma$ that describes the depth $O\left(\varepsilon^{\gamma}\right)$ of irregularity ( $\varepsilon$ is the oscillation period). The resulting formulas relate the eigenvalues in domains with close irregular boundaries and make it possible, in particular, to control the order of perturbation and to find conditions ensuring the validity (or violation) of the classical Hadamard formula.
\end{abstract}

\section{§1. INTRODUCTION}

1. Setting of problems. Let a domain $\Omega$ on the plane $\mathbb{R}^{2}$ be bounded by a simple, closed, and smooth (of class $C^{\infty}$ ) contour $\Gamma=\partial \Omega$. By scaling, we make the length of $\Gamma$ equal to 1 . In a neighborhood $\mathcal{V}$ of $\Gamma$ we introduce a system of natural curvilinear coordinates $(n, s)$, where $n$ is the distance to $\Gamma$, taken with the minus sign inside of $\Omega$, and $s$ is the arclength on $\Gamma$. The rapidly oscillating boundary $\Gamma^{\varepsilon}$ of the perturbed domain $\Omega^{\varepsilon}$ (see Figure 1) is defined by the formula

$$
\Gamma^{\varepsilon}=\left\{x \in \mathcal{V}: s \in \Gamma, n=\varepsilon^{\gamma} H\left(\varepsilon^{-1} s, s\right)\right\},
$$

where $\varepsilon=1 / N$ is a small parameter, $N \in \mathbb{N}$ is a large integer, $\gamma$ is a quantity measuring the "irregularity" of the boundary (the greater is $\gamma$, the smaller is the perturbation irregularity), and $H$ is a profile function that is smooth relative to both variables, the "slow" variable $s$ and the "fast" variable $\eta=\varepsilon^{-1} s$, and 1-periodic relative to $\eta$. Note that, somewhat loosely, in our notation we do not distinguish between a point $s \in \Gamma$ and its coordinate. For the role of $\mathcal{V}$ it is convenient to take the $\varrho$-neighborhood $\mathcal{V}_{\varrho}$ with an appropriate $\varrho>0$.

Concerning the nonregular perturbation of the boundary (see Figure 2), we assume that

$$
\Gamma \in \operatorname{Lip}, \quad \Delta\left(\Omega, \Omega^{\varepsilon}\right):=\left(\Omega \backslash \Omega^{\varepsilon}\right) \cup\left(\Omega^{\varepsilon} \backslash \Omega\right) \subset \mathcal{V}_{\varepsilon} .
$$

In all other respects, the perturbation of $\Omega$ is arbitrary. To avoid numerous duplication of formulas, we keep the notation $\Omega$ and $\Omega^{\varepsilon}$ also in $\S 4$. It should be emphasized that

2010 Mathematics Subject Classification. Primary 35J40, Secondary 31A25, 31A30, 35P20.

Key words and phrases. Biharmonic operator, Dirichlet problem, asymptotic expansions of eigenvalues, eigenoscillations of the Kirchhoff plate, rapid oscillation and nonregular perturbation of the boundary.

The paper was written during S. A. Nazarov's visit to the University of Linköping, whose financial support is acknowledged gratefully. Also, V. A. Kozlov thanks the Swedish Research Council (VR), and S. A. Nazarov thanks RFBR (project no. 09-01-00759). 


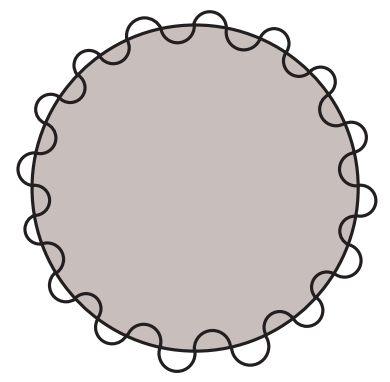

Figure 1. A domain with rapidly oscillating boundary. The initial domain is tinted.

the calculations and arguments of $\S 4$ remain valid for multidimensional domains, but the authors know of no physical model involving a fourth-order differential equation in $\mathbb{R}^{d}$, $d \geq 3$, so that we pay no attention to this possibility.

2. The problem in a domain with rapidly oscillating boundary. We return to discussing the locally periodic boundary (1.1). In the domain $\Omega^{\varepsilon}$ we consider the spectral problem 1

$$
\begin{aligned}
\Delta_{x}^{2} u^{\varepsilon}(x) & =\lambda^{\varepsilon} u^{\varepsilon}(x), \quad x \in \Omega^{\varepsilon}, \\
u^{\varepsilon}(x) & =0, \quad \partial_{n^{\varepsilon}} u^{\varepsilon}(x)=0, \quad x \in \Gamma^{\varepsilon} .
\end{aligned}
$$

Here $\Delta_{x}$ is the Laplace operator in the Cartesian coordinates $x=\left(x_{1}, x_{2}\right), \lambda^{\varepsilon}$ is the spectral parameter, and $\partial_{n^{\varepsilon}}$ stands for the derivative along the outward normal to the boundary $\Gamma^{\varepsilon}=\partial \Omega^{\varepsilon}$ of the perturbed domain $\Omega^{\varepsilon}$. The variational setting of problem (1.3), (1.4) involves the integral identity [1]

$$
\left(\Delta_{x} u^{\varepsilon}, \Delta_{x} v^{\varepsilon}\right)_{\Omega^{\varepsilon}}=\lambda^{\varepsilon}\left(u^{\varepsilon}, v^{\varepsilon}\right)_{\Omega^{\varepsilon}}, \quad v^{\varepsilon} \in \stackrel{\circ}{H}^{2}\left(\Omega^{\varepsilon}\right),
$$

in which $(,)_{\Omega^{\varepsilon}}$ is the natural scalar product in the Lebesgue space $L_{2}\left(\Omega^{\varepsilon}\right)$, and $\stackrel{\circ}{H}^{2}\left(\Omega^{\varepsilon}\right)$ denotes the subspace of the Sobolev space $H^{2}\left(\Omega^{\varepsilon}\right)$ formed by the functions satisfying the boundary conditions (1.4). The second and first basic inequalities (see [1, Chapter 2]) imply the relation

$$
\left\|u^{\varepsilon} ; H^{2}\left(\Omega^{\varepsilon}\right)\right\| \leq c\left\|\Delta_{x} u^{\varepsilon} ; L_{2}\left(\Omega^{\varepsilon}\right)\right\|, \quad u^{\varepsilon} \in \stackrel{\circ}{H}^{2}\left(\Omega^{\varepsilon}\right),
$$

and the constant $c$ can be taken to serve all $\varepsilon \in\left(0, \varepsilon_{0}\right]$ (the function $u^{\varepsilon}$ can be extended by zero to a larger fixed domain). The left-hand side of (1.5) is a scalar product in the Hilbert space $\stackrel{\circ}{H}^{2}\left(\Omega^{\varepsilon}\right)$, and thus, by [2, Theorems 10.1.5 and 10.2.2], the spectrum of problem (1.5) is discrete, and the eigenvalues form an unbounded monotone sequence

$$
0<\lambda_{1}^{\varepsilon} \leq \lambda_{2}^{\varepsilon} \leq \cdots \leq \lambda_{p}^{\varepsilon} \leq \cdots \rightarrow+\infty,
$$

in which they are counted with regard to multiplicity, and the corresponding eigenfunctions $u_{1}^{\varepsilon}, u_{2}^{\varepsilon}, \ldots, u_{p}^{\varepsilon}, \ldots \in H^{2}\left(\Omega^{\varepsilon}\right)$ can be taken to obey the orthogonality and normalization conditions

$$
\left(u_{p}^{\varepsilon}, u_{q}^{\varepsilon}\right)_{\Omega^{\varepsilon}}=\delta_{p, q}, \quad p, q \in \mathbb{N} .
$$

Here $\delta_{p, q}$ is the Kronecker symbol. The boundary-value problem (1.3), (1.4) is known as the Kirchhoff model of the bending oscillations of a thin plate with a clamped edge

\footnotetext{
${ }^{1}$ In fact, we deal with a family of problems parametrized by the quantity $\varepsilon=1 / N$, but it is convenient to assume the parameter to be small but fixed and name the accompanying objects in the singular.
} 


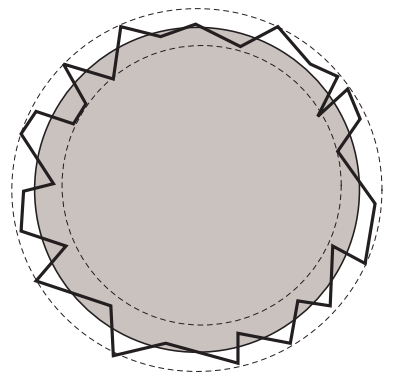

FiguRE 2. A domain with irregularly perturbed boundary.

(see [3, §30] and 4, Chapter 4]), and equation (1.3) is named after Sophie Germain? Besides the flat roofs of modern arenas and other sizable buildings, this problem describes deformations of the ice covers of water bodies with a highly indented coastline, for example, of Norwegian fiords, which do not freeze up at the present time, but can lose this advantageous property due to changes in the Gulf Stream parameters, as some unfavorable predictions of geophysicists and meteorologists say.

In the homogenization theory, as applied to the boundary-value problem in the domain $\Omega^{\varepsilon}$ bounded by the contour (1.1), a main goal is to characterize the behavior of the eigenvalues $\lambda_{p}^{\varepsilon}$ as $\varepsilon \rightarrow+0$ in dependence of the exponent $\gamma$ in (1.1) (Theorem 3.1) and to describe the "smooth picture" of the rapidly oscillating boundary $\Gamma^{\varepsilon}$ (see Subsection 3 in $\S 5$ ).

If the boundary perturbation is periodic, then the following simple claim about convergence provides the "limiting" problem

$$
\begin{aligned}
\Delta_{x}^{2} u(x) & =\lambda u(x), \quad x \in \Omega, \\
u(x) & =\partial_{n} u(x)=0, \quad x \in \Gamma,
\end{aligned}
$$

or, more precisely, its variational formulation

$$
\left(\Delta_{x} u, \Delta_{x} v\right)_{\Omega}=\lambda(u, v)_{\Omega}, \quad v \in \stackrel{\circ}{H}^{2}(\Omega) .
$$

Lemma 1.1. The limit $\lambda_{q}^{0}=\lim \lambda_{q}^{\varepsilon}$ as $\varepsilon \rightarrow+0$ along, possibly, some infinitesimally small sequence, belongs to the collection

$$
0<\lambda_{1} \leq \lambda_{2} \leq \cdots \leq \lambda_{p} \leq \cdots \rightarrow+\infty
$$

of eigenvalues of problem (1.11). The corresponding eigenfunctions, extended by zero outside of $\Omega^{\varepsilon}$, converge in $L_{2}\left(\mathbb{R}^{2}\right)$ to some eigenfunction $u_{q}^{0} \in \stackrel{\circ}{H}^{2}(\Omega)$ of problem (1.5), and $\left\|u_{q}^{0} ; L_{2}(\Omega)\right\|=1$.

On its own, the limiting problem (1.9), (1.10) carries no information on the degree of irregularity of $\Gamma^{\varepsilon}$; therefore, a key role is played by the correction term in the eigenvalue's asymptotic expansion. If the boundary is perturbed regularly (the profile $H$ is independent of the fast variable; see Subsection 1 in $\S 2$ and Figure 3), for the simple eigenvalue

\footnotetext{
${ }^{2}$ In 1815 , for the deduction of the equation for eigenoscillations of plates, mademoiselle Sophie Germain was awarded a prize of French Academy. Afterwords, S. Poisson found a mistake in her calculations and published corrections in 1829. A complete theory of plate bending, based on an empiric asymptotic analysis, was created by Kirchhoff in 1850-1859.
} 


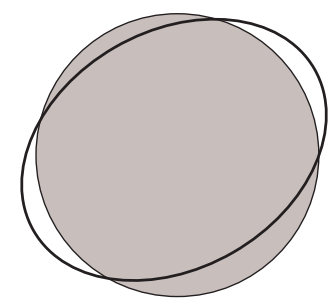

FIGURE 3. A domain with a regularly perturbed boundary.

$\lambda_{p}$ the asymptotic formula, whose proper name should be the Hadamard formula [5], takes the form

$$
\lambda_{p}^{\varepsilon}=\lambda_{p}+\varepsilon^{\gamma} \int_{\Gamma} H(s)\left|\partial_{n}^{2} u_{p}(0, s)\right|^{2} d s+\tilde{\lambda}_{p}^{\varepsilon} .
$$

Note that when using curvilinear coordinates, we write $u_{p}(n, s)$ as before. We have $\partial_{n}^{2} u_{p}(0, s)=\Delta_{x} u_{p}(0, s)$ by the boundary conditions on $\Gamma$, because

$$
\Delta_{x}=J(n, s)^{-1}\left(\partial_{n} J(n, s) \partial_{n}+\partial_{s} J(n, s)^{-1} \partial_{s}\right),
$$

where $J(n, s)=1+n \kappa(s)$ is the Jacobian, and $\kappa(s)$ is the curvature of $\Gamma$ at the point $s$. Finally, the remainder term $\tilde{\lambda}_{p}^{\varepsilon}$ in (1.13) is an infinitesimally small quantity of order of $\varepsilon^{2 \gamma}$.

If $\gamma>1$, i.e., the boundary perturbation remains slanting, then the Hadamard formula (1.13) keeps its form, but the factor $H(s)$ in the integrand should be replaced with the mean value

$$
\bar{H}(s)=\int_{0}^{1} H(\eta, s) d \eta,
$$

and the estimate for the remainder term needs modification.

In the case of $\gamma \leq 1$, where the boundary becomes highly indented, the structure of the asymptotic behavior changes substantially: the phenomenon of a boundary layer arises, and the weight factor in the integral in (1.13) acquires a radically different meaning; namely, it can be expressed in terms of some coefficients in asymptotic expansions at infinity of certain special solutions of auxiliary boundary-value problems in a half-strip with curvilinear edge or in a strip with a semi-infinite cut. In this case, the size $O\left(\varepsilon^{\gamma}\right)$ of the perturbation, proportional to the depth of the hollows and (or) the height of the ridges, always remains unchanged, with one exception: if $\gamma<1$ and

$$
H_{0}(s)=\min _{\eta \in[0,1)} H(\eta, s)=0,
$$

then the correction term is of order of $\varepsilon$, rather than $\varepsilon^{\gamma}$, as the Hadamard formula predicts.

3. Summary of results on nonregular perturbation of the boundary. Consider the spectral problems (1.11) and (1.5) in the domains $\Omega$ and $\Omega^{\varepsilon}$ satisfying (1.2). Let $\lambda_{p}$ be an eigenvalue of (1.11) of multiplicity $\varkappa_{p}$, i.e., in (1.12) we have

$$
\lambda_{p-1}<\lambda_{p}=\cdots=\lambda_{p+\varkappa_{p}-1}<\lambda_{p+\varkappa_{p}} .
$$


The corresponding eigenspace $\mathfrak{X}_{p}$ is spanned by the eigenfunctions $u_{p}, \ldots, u_{p+\varkappa_{p}-1}$. It is known (see, e.g., [6, 7, and compare to Lemma 1.1) that for small $\varepsilon>0$ on the interval

$$
\left(\frac{1}{2}\left(\lambda_{p-1}+\lambda_{p}\right), \frac{1}{2}\left(\lambda_{p}+\lambda_{p+\varkappa_{p}}\right)\right)
$$

there are precisely the eigenvalues $\lambda_{p}^{\varepsilon}, \ldots, \lambda_{p+\varkappa_{p}-1}^{\varepsilon}$ of problem (1.5).

In what follows we shall see that these eigenvalues satisfy

$$
\left(\lambda_{q}^{\varepsilon}\right)^{-1}=\lambda_{p}^{-1}+\vartheta_{q}^{\varepsilon}+O\left(\varepsilon^{1+\delta}\right), \quad q=p, \ldots, p+\varkappa_{p}-1,
$$

where $\delta$ is a positive quantity depending on $\Omega$, and $\vartheta_{p}^{\varepsilon}, \ldots, \vartheta_{p+\varkappa_{p}-1}^{\varepsilon}$ are eigenvalues of the algebraic problem (posed on a finite-dimensional subspace)

$$
\begin{array}{r}
\lambda_{p}^{-1}\left(\left(\Delta_{x} \Psi_{\varphi}^{\varepsilon}, \Delta_{x} \Psi_{\psi}^{\varepsilon}\right)_{\Omega^{\varepsilon}}-\left(\Delta_{x} \Phi_{\varphi}^{\varepsilon}, \Delta_{x} \Phi_{\psi}^{\varepsilon}\right)_{\Omega^{\varepsilon}}-\left(\Delta_{x} \varphi, \Delta_{x} \psi\right)_{\Omega \backslash \Omega^{\varepsilon}}\right) \\
=\vartheta\left(\Delta_{x} \varphi, \Delta_{x} \psi\right)_{\Omega}, \quad \psi \in \mathfrak{X}_{p},
\end{array}
$$

for $\varphi \in \mathfrak{X}_{p}$. Here, $\Phi_{\varphi}^{\varepsilon}$ is the solution of the boundary-value problem (without spectral parameter) in $\Omega^{\varepsilon}$, formulated as the integral identity

$$
\left(\Delta_{x} \Phi_{\varphi}^{\varepsilon}, \Delta_{x} v\right)_{\Omega^{\varepsilon}}=0, \quad v \in \stackrel{\circ}{H}^{2}\left(\Omega^{\varepsilon}\right),
$$

and also $\Phi_{\varphi}^{\varepsilon}+\varphi \in \stackrel{\circ}{H}^{2}\left(\Omega^{\varepsilon}\right)$. The function $\Psi_{\varphi}^{\varepsilon} \in \stackrel{\circ}{H}^{2}\left(\Omega^{\varepsilon}\right)$ obeys another integral identity

$$
\left(\Delta_{x} \Psi_{\varphi}^{\varepsilon}, \Delta_{x} v\right)_{\Omega^{\varepsilon}}=\left(\Delta_{x} \varphi, \Delta_{x} v\right)_{\Omega^{\varepsilon}}-\lambda_{p}(\varphi, v)_{\Omega^{\varepsilon}}, \quad v \in \stackrel{\circ}{H}^{2}\left(\Omega^{\varepsilon}\right) .
$$

Note that if $u_{q} \in \stackrel{\circ}{H}^{4}(\Omega)$ (e.g., $\Gamma$ is smooth), then the right-hand side of (1.22) is equal to the surface integral $\left(\Delta_{x} \varphi, \partial_{n} v\right)_{\Gamma}-\left(\partial_{\nu} \Delta_{x} \varphi, v\right)_{\Gamma}$.

Now we state yet another result obtained in $\S 4$. Suppose that the boundary $\Gamma$ of the domain $\Omega$ is a surface of Hölder class $C^{1, \alpha}, \alpha \in(0,1)$. On $\Gamma$, we introduce the function $h^{\varepsilon}$ equal to the distance to $\Gamma^{\varepsilon}$ along the normal $\vec{n}$ at the point $s$. In Subsection 7 of $\S 4$ we shall show that under the restriction

$$
\int_{\Gamma}\left|\nabla_{s} h^{\varepsilon}(s)\right|^{2} d s=o(1), \quad \varepsilon \rightarrow+0,
$$

we have the formula

$$
\left(\lambda_{q}^{\varepsilon}\right)^{-1}=\lambda_{p}^{-1}+\theta_{q}+o(\varepsilon), \quad q=p, \ldots, p+\varkappa_{p}-1,
$$

where $\theta_{p}, \ldots, \theta_{p+\varkappa_{p}-1}$ are eigenvalues of the following problem algebraic on the subspace $\mathfrak{X}_{p}$ :

$$
\lambda_{p}^{-1}\left(h \Delta_{x} \varphi, \Delta_{x} \psi\right)_{\Gamma}=\theta\left(\Delta_{x} \varphi, \Delta_{x} \psi\right)_{\Omega}, \quad \psi \in \mathfrak{X}_{p}
$$

(cf. problem (1.20)).

Relation (1.24) can be understood as a generalization of the Hadamard formula; in particular, for a simple eigenvalue, i.e., for $\varkappa_{p}=1$, it is not hard to reshape (1.24) to (1.13) with $\gamma=1$. It should be emphasized that now we do not deal with any periodicity of the boundary perturbation whatever.

In Subsection 8 of $\S 4$, for a 2-dimensional domain $\Omega^{\varepsilon} \subset \Omega$ with corner points on the boundary, we show that if one of the angles exceeds $\pi$, so that $\Omega$ cannot be convex, then the Hadamard formula fails. However, relation (1.24) remains valid provided that $\theta_{q}, \ldots, \theta_{p+\varkappa_{p}-1}$ are found from the spectral problem

$$
\lambda_{p}^{-1}\left(\Delta_{x} \varphi, \Delta_{x} \psi\right)_{\Omega \backslash \Omega^{\varepsilon}}=\theta\left(\Delta_{x} \varphi, \Delta_{x} \psi\right)_{\Omega}, \quad \psi \in \mathfrak{X}_{p},
$$

rather than from (1.25). Note that on the left in (1.26) we have a volume integral, while in (1.25) we have a surface one; if $\Gamma$ is smooth, then these integrals coincide up to $O(\varepsilon)$. From this viewpoint, the method of writing the Hadamard formula via volume integrals, as suggested in [7, appears more unified and universal. 
4. Plan of the paper and a brief review of publications. In the next section we are focused on the formal asymptotic analysis of the spectral problem (1.3), (1.4) in a domain with a rapidly oscillating boundary (1.1) (see Figure 1). We apply the methods of composite and matching asymptotic expansions (see, e.g., 9] and [10], respectively). The formulas obtained are justified in $\S 3$.

There are numerous publications devoted to the study of various boundary-value problems in domains with locally periodic boundaries (see, e.g., [11, §7.5] and [12]-[15] for scalar equations, [16- 18, for the Stokes system, and [19-21] for the elasticity theory system); however, the results pertain largely to the case of $\gamma=1$, where the period, the depth of the hollows, and the height of the ridges are of one and the same order. The papers that address the case of $\gamma=0$ (we do not consider this case in the present paper; cf. Remark 5.1) deal with the Neumann boundary conditions, for which the structure of the spectrum is very specific.

Usually, the "support" boundary $\Gamma$ is assumed to be smooth, and there are only several publications [26]-30] where the impact of the boundary peculiarities was taken into account, namely, of the presence of corner points on $\Gamma$, which results in the arising of a 2-dimensional boundary layer.

In a more general situation where the initial and perturbed boundaries are Lipschitzian, some formulas that enable one to compare the eigenvalues of the Dirichlet problems for the Laplace operators in two domains were obtained in [7] on the basis of a general abstract method; see [7, 8] (for other approaches to studying the spectra of these two scalar problems, see the books [34, 33] and the references therein). In $\S 4$, the approach of 7 is applied to the investigation of the Dirichlet problem for the biharmonic operator. As could be expected in the setting of arbitrary perturbations, the final formulas (1.19) and (1.20) turn out to be not so explicit, requiring a further elaboration in concrete situations, but in Subsections 7 and 8 of $\S 4$ and in Subsection 1 of $\S 5$ we show that under some higher smoothness assumptions this formula leads to the same results as in $\S 3$.

In $\S 5$ we discuss possible generalizations of our asymptotic procedures.

The general results of [7. allow us to simplify the justification of the asymptotic procedure, because formulas (1.19) and (1.20) deal with solutions $\Phi_{\varphi}^{\varepsilon}$ and $\Psi_{\varphi}^{\varepsilon}$ of the boundary-value problems (1.21) and (1.22) that are free of the spectral parameter, i.e., admit simpler asymptotic analysis. In general, justification of the asymptotic methods for fourth-order equations in domains with rapidly oscillating boundary, as presented in $\S 3$, is more intricate compared to the case of an equation or a system of order two. The reason is that, in construction of the global asymptotic approximation (Subsection 3 of $\S 3)$, the first boundary condition (1.4) is often fulfilled automatically, but the second boundary condition gives rise to a discrepancy that must be compensated.

The asymptotic formulas obtained turn out to be useful also in the theory of optimization of shapes (see [33-36] and elsewhere). We note that the absolute majority of issues, studied fully in the case of the Dirichlet problem for the Laplace operator, remain open for problem (1.3), (1.4) (see [37-39]).

Also, it should be noted that the biharmonic equation with simple support boundary conditions (see [40, 3]) in a domain with a singularly perturbed boundary gives rise to "unexpected" asymptotic formulas known as the paradoxes of the theory of plates (see, e.g., [41-43]).

In the paper, we separate two approaches to finding the asymptotic expansions of eigenvalues. Therefore, the reader interested in results traditional in homogenization theory can skip $\S 4$ and Subsection 1 of $\S 5$ (precisely in order to ensure this possibility, in Subsection 2 of $\S 3$ we present the proof of the simple Lemma 1.1, which follows from 
the general results; see Subsection 3 of $\S 1$ ). On the other hand, the description of the function theory approach is concentrated in $\S 4$, and in Subsection 1 of $\S 5$ we discuss its relationship with the asymptotic approach.

Throughout, the letter $c$ denotes a factor in majorants independent of the parameter $\varepsilon \in\left(0, \varepsilon_{0}\right]$; this $c$ can vary from line to line even in one formula. The upper bound $\varepsilon_{0}$ is also not fixed, remaining always positive.

\section{§2. Formal asymptotic analysis in the CASE of A PERIODIC BOUNDARY PERTURBATION}

1. Asymptotic analysis in the case of a slanting boundary perturbation. First, we suppose that the profile function $H$ in formula (1.1) does not depend on the fast variable $\eta=\varepsilon^{-1} s$. Taking an eigenvalue $\lambda_{p}$ of the limiting problem (1.9), (1.10), we denote by $\varkappa_{p}$ its multiplicity; then (1.17) is valid. We assume the following asymptotic Ansatz for the solutions of the perturbed problem (1.3), (1.4):

$$
\begin{aligned}
\lambda_{q}^{\varepsilon} & =\lambda_{p}+\varepsilon^{\gamma} \lambda_{q}^{\prime}+\tilde{\lambda}_{q}^{\varepsilon}, \\
u_{q}^{\varepsilon}(x) & =u_{q}^{0}(x)+\varepsilon^{\gamma} u_{q}^{\prime}(x)+\widetilde{u}_{q}^{\varepsilon}(x)
\end{aligned}
$$

here $q=p, \ldots, p+\varkappa_{p}-1$, and $u_{q}^{0}$ is a linear combination of eigenfunctions $u_{p}, \ldots, u_{p+\varkappa_{p}-1}$ of problem (1.9), (1.10) that correspond to the eigenvalue $\lambda_{p}$. In other words,

$$
u_{q}^{0}=\sum_{m=p}^{p+\varkappa_{p}-1} a_{m}^{q} u_{m}, \quad \sum_{m=p}^{p+\varkappa_{p}-1}\left|a_{m}^{q}\right|^{2}=1,
$$

and, if necessary (i.e., if $\Omega^{\varepsilon} \backslash \bar{\Omega} \neq \varnothing$ ), the $u_{m}$ are assumed to be smoothly extended outside $\Omega$. Of course, as in (1.8), the eigenfunctions are subject to the orthogonality and normalization conditions

$$
\left(u_{j}, u_{k}\right)_{\Omega}=\delta_{j, k}, \quad j, k \in \mathbb{N} .
$$

We write the leading asymptotic term $u_{q}^{0}$ in curvilinear coordinates and apply the Taylor formula; using the boundary conditions (1.10), we get

$$
u_{q}^{0}(n, s)=\frac{1}{2} n^{2} \partial_{n}^{2} u_{q}^{0}(0, s)+O\left(n^{3}\right) .
$$

Thus, the discrepancy of the function $u_{q}^{0}$ in the first identity in (1.4),

$$
u^{\varepsilon}\left(\varepsilon^{\gamma} H\left(\varepsilon^{-1} s, s\right), s\right)=0, \quad s \in \Gamma,
$$

is $O\left(\varepsilon^{2 \gamma}\right)$, so that the order of it is higher than that of the correction terms in (2.1) and (2.2).

Now we calculate discrepancies in the second boundary condition

$$
\partial_{n^{\varepsilon}} u^{\varepsilon}\left(\varepsilon^{\gamma} H\left(\varepsilon^{-1} s, s\right), s\right)=0, \quad s \in \Gamma ;
$$

recall that $\partial_{n^{\varepsilon}}$ denotes the derivative along the outward normal to the oscillating boundary $\Gamma^{\varepsilon}$. In the neighborhood $\mathcal{V}$, the gradient operator can be written as

$$
\nabla_{x}=e_{(n)} \partial_{n}+J(n, s)^{-1} e_{(s)} \partial_{s},
$$

where $e_{(n)}$ and $e_{(s)}$ are the unit vectors of the axes $n$ and $s$, and $J(n, s)$ is the Jacobian (see (1.14)). Consequently, the normal and the derivative along it take the form

$$
\begin{aligned}
n^{\varepsilon}(s) & =\left(1+N^{\varepsilon}(s)^{2}\right)^{-1 / 2}\left(e_{(n)}-N^{\varepsilon}(s) e_{(s)}\right), \\
\partial_{n^{\varepsilon}} & =\left(1+N^{\varepsilon}(s)^{2}\right)^{-1 / 2}\left(\partial_{n}-N^{\varepsilon}(s) J^{\varepsilon}(s)^{-1} \partial_{s}\right),
\end{aligned}
$$


and

$$
\begin{aligned}
J^{\varepsilon}(s) & =1+\varepsilon^{\gamma} H\left(\varepsilon^{-1} s, s\right) \varkappa(s), \\
N^{\varepsilon}(s) & =\left.\varepsilon^{\gamma} J^{\varepsilon}(s)\left(\partial_{s} H(\eta, s)+\varepsilon^{-1} \partial_{\eta} H(\eta, s)\right)\right|_{\eta=\varepsilon^{-1} s} .
\end{aligned}
$$

If $H$ is independent of the fast variable, then $J^{\varepsilon}(s)=1+O\left(\varepsilon^{\gamma}\right)$ and $N^{\varepsilon}(s)=O\left(\varepsilon^{\gamma}\right)$, so that the derivatives $\partial_{n^{\varepsilon}}$ and $\partial_{n}$ differ little. As a result, to compensate the leading term of the discrepancy in relation (2.6), we can take a function $u_{q}^{\prime}$ satisfying

$$
u_{q}^{\prime}(0, s)=0, \quad \partial_{n} u_{q}^{\prime}(0, s)=-H(s) \partial_{n}^{2} u_{q}^{0}(0, s), \quad s \in \Gamma .
$$

Now we plug (2.1) and (2.2) into the differential equation (1.3); collecting the factors of $\varepsilon^{\gamma}$, we get

$$
\Delta_{x}^{2} u_{q}^{\prime}(x)-\lambda_{p} u_{q}^{\prime}(x)=\lambda_{q}^{\prime} u_{q}^{0}(x), \quad x \in \Omega .
$$

Since $\lambda_{p}$ is an eigenvalue of problem (1.9), (1.10) with multiplicity, the resulting boundary-value problem (2.12), (2.11) has a solution if and only if the following $\varkappa_{p}$ solvability conditions are fulfilled:

$$
\begin{aligned}
\lambda_{q}^{\prime}\left(u_{q}^{0}, u_{j}\right)_{\Omega} & =\left(\Delta_{x}^{2} u_{q}^{0}-\lambda_{p} u_{q}^{0}, u_{j}\right)_{\Omega}=\left(\partial_{n} u_{q}^{\prime}, \Delta_{x} u_{j}\right)_{\Gamma} \\
& =-\left(H \partial_{n}^{2} u_{q}^{0}, \Delta_{x} u_{j}\right)_{\Gamma}, \quad j=p, \ldots, p+\varkappa_{p}-1 .
\end{aligned}
$$

Formula (2.4) with the Jacobian $J(n, s)=1+n \kappa(s)$, which is equal to 1 on $\Gamma$, and the boundary conditions (1.10) show that $\Delta_{x} u_{j}(0, s)=\partial_{n}^{2} u_{j}(0, s)$. Therefore, by (1.14), relations (2.13) can be rewritten as the system

$$
\mathbf{T}^{p} a^{q}=\lambda_{q}^{\prime} a^{q}
$$

of $\varkappa_{p}$ algebraic equations, where $a^{q}=\left(a_{1}^{q}, \ldots, a_{\varkappa_{p}}^{q}\right)$ is the column of coefficients occurring in (2.3), and $\mathbf{T}^{p}$ is the symmetric $\left(\varkappa_{p} \times \varkappa_{p}\right)$-matrix with the elements

$$
\mathbf{T}_{j k}^{p}=-\int_{\Gamma} h(s) \partial_{n}^{2} u_{j}(0, s) \partial_{n}^{2} u_{k}(0, s) d s, \quad j, k=p, \ldots, p+\varkappa_{p}-1,
$$

and in the case under consideration we have $h(s)=H(s)$. The eigenvalues of the matrix $\mathbf{T}^{p}$ will be enumerated in the nondecreasing order, i.e.,

$$
\lambda_{p}^{\prime} \leq \cdots \leq \lambda_{p+\varkappa_{p}-1}^{\prime},
$$

and the eigenvectors will satisfy the conditions of normalization (the second formula in (2.3) ) and orthogonality

$$
\sum_{j=p}^{p+\varkappa_{p}-1} a_{j}^{q} a_{j}^{m}=0, \quad q \neq m .
$$

The eigenvalues $\lambda_{p}^{\prime}, \ldots, \lambda_{p+\varkappa_{p}-1}^{\prime}$ of $\mathbf{T}^{p}$ and the eigenvectors $a^{p}, \ldots, a^{p+\varkappa_{p}-1}$ of unit length corresponding to them complete the expansions (2.1) and (2.2), but the solution $u_{q}^{\prime}$ of problem (2.12), (2.11) still has freedom, namely, the linear combination of $u_{p}, \ldots, u_{p+\varkappa_{p}-1}$.

We return to the case of a rapidly oscillating boundary (1.1); i.e., we recover the dependence of the profile function $H$ on the first argument. The quantity (2.10) is $O\left(\varepsilon^{\gamma-1}\right)$, so that the conclusion is that it is small and the subsequent calculations remain valid in the case where $\gamma>1$, which corresponds, as before, to a slanting perturbation of the boundary. It is not hard to check the following estimate, which is widely used in homogenization theory:

$$
\left|\int_{\Gamma}\left(H\left(\varepsilon^{-1} s, s\right)-\bar{H}(s)\right) Y(s) d s\right| \leq c \varepsilon\left\|Y ; C^{1}(\Gamma)\right\| \max _{s \in \Gamma, \eta \in[0,1)}|H(\eta, s)|,
$$




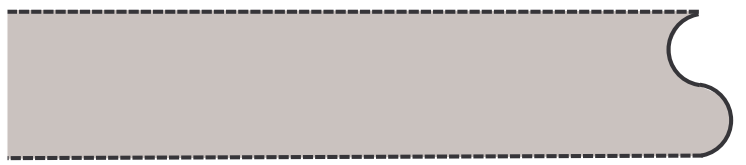

FIGURE 4. The half-strip $\Pi$ with curvilinear edge $\varpi$.

where $\bar{H}$ is the averaged profile function (1.15). Therefore, the final formulas (2.14) and (2.15) require no change, but the role of the weight factor is played by the averaged profile function (1.15), i.e.,

$$
h(s)=\bar{H}(s) .
$$

2. The boundary layer and asymptotic expansions for $\gamma=1$. There are many publications devoted to the study of solutions of boundary-value problems in domains with rapidly oscillating boundaries such that the oscillation period and the erosion depth have the same order of smallness (see, e.g., [11, 12, 15]). The resulting asymptotic formulas are characterized, first of all, by the boundary layer phenomenon described in terms of the dilated coordinates

$$
\xi=\left(\xi_{1}, \xi_{2}\right), \quad \xi_{1}=\varepsilon^{-1} n, \quad \xi_{2}=\varepsilon^{-1} s .
$$

This boundary layer is constructed from 1-periodic (in $\xi_{2}$ ) solutions of boundary-value problems in a half-strip $\Pi(s)$ with curvilinear edge $\varpi(s)$ (see Figure 4). In accordance with (1.1) and (2.19), we have

$$
\Pi(s)=\left\{\xi: \xi_{2} \in(0,1), \xi_{1}<H\left(\xi_{2}, s\right)\right\}, \varpi(s)=\left\{\xi: \xi_{2} \in(0,1), \xi_{1}=H\left(\xi_{2}, s\right)\right\} .
$$

The problem for the boundary layer type terms looks like this:

$$
\begin{aligned}
\Delta_{\xi}^{2} w(\xi ; s) & =0, \quad \xi \in \Pi(s), \\
w(\xi ; s) & =g_{0}(\xi ; s), \quad \partial_{\nu} w(\xi ; s)=g_{1}(\xi ; s), \quad \xi \in \varpi(s), \\
w\left(\xi_{1}, 0 ; s\right) & =w\left(\xi_{1}, 1 ; s\right), \quad \partial_{2} w\left(\xi_{1}, 0 ; s\right)=\partial_{2} w\left(\xi_{1}, 1 ; s\right), \quad \xi_{1}<H(0, s) .
\end{aligned}
$$

Here $\partial_{j}=\partial / \partial \xi_{j}, j=1,2$. The origin of the boundary conditions (2.22) is clear: in their right-hand sides we have the discrepancies left by the asymptotic term (2.3) of regular type. The biharmonic equation (2.21) arises as a result of the coordinate changes $x \mapsto(n, s) \mapsto \xi$ and formula (1.14) for the Laplace operator in curvilinear coordinates. Finally, the periodicity conditions (2.23) on the lateral sides of the half-strip (the dashdot lines in Figure 4) are determined by the construction itself of the boundary layer that occurs in the modified (compared to (2.2) ) representation of the eigenfunction

$$
u_{q}^{\varepsilon}(x)=u_{q}^{0}(x)+\chi(x) \varepsilon^{2} w_{q}\left(\varepsilon^{-1} n, \varepsilon^{-1} s ; s\right)+\varepsilon u_{q}^{\prime}(x)+\widetilde{u}_{q}^{\varepsilon}(x) .
$$

Here $\chi$ is a smooth cutoff function supported on the neighborhood $\mathcal{V}$ and equal to 1 near $\Gamma$. The data of problem (2.21)-(2.23) depend on the parameter $s \in \Gamma$, but we shall not indicate this in what follows if there is no danger of confusion.

The next claim is a specialization of the general results obtained in [44, §4] on the basis of the theory of elliptic boundary-value problems in domains with cylindrical exits to infinity (see the key papers [45, 46, 47, 48, and also, e.g., the books 49, 50]).

Proposition 2.1. 1) Any solution of the homogeneous $\left(g_{0}=g_{1}=0\right)$ problem (2.21)(2.23) with at most polynomial growth at infinity is a linear combination of two solutions 
determined by the asymptotic expansions

$$
\begin{aligned}
& W_{0}(\xi ; s)=\frac{1}{2} \xi_{1}^{2}-P_{00}(s) \xi_{1}-P_{01}(s)+O\left(\exp \left(\delta \xi_{1}\right)\right), \\
& W_{1}(\xi ; s)=\frac{1}{6} \xi_{1}^{3}-P_{10}(s) \xi_{1}-P_{11}(s)+O\left(\exp \left(\delta \xi_{1}\right)\right),
\end{aligned}
$$

where the $P_{i l}(s)$ and $\delta>0$ are some numbers. The quantities $W_{i}(\xi ; s)$ and $P_{i l}(s)$ depend smoothly on $s \in \Gamma$.

2) Problem (2.21)-(2.23) with smooth periodic right-hand sides $g_{0}$ and $g_{1}$ admits a solution $w$ decaying exponentially as $\xi_{1} \rightarrow-\infty$ if and only if the following two solvability conditions are satisfied:

$$
\int_{\varpi(s)} g_{0}(\xi) \partial_{\nu} \Delta_{\xi} W_{i}(\xi) d s_{\xi}-\int_{\varpi(s)} g_{1}(\xi) \Delta_{\xi} W_{i}(\xi) d s_{\xi}=0, \quad i=0,1 .
$$

Such a solution $w$ is unique and inherits smoothness relative to $s$ from the right-hand sides $g_{0}$ and $g_{1}$.

In the sequel, our asymptotic constructions will involve only the solution $W_{0}$, and the coefficient $P_{00}$, denoted simply by $P$, arises in a formula of the type (1.13) for eigenvalues. The periodicity conditions (2.23) and the smoothness of the data show that the representations (2.25) admit termwise differentiation, with preservation of the rate of decay of the remainder term.

Proposition 2.2. If $H(\eta, s)=H(s)$ is constant, then $P(s):=P_{00}(s)=H(s)$. If the profile function $\eta \mapsto H(\eta, s)$ is nonconstant, then we have the inequalities

$$
H_{0}(s):=\min _{\eta \in[0,1)} H(\eta, s)<P(s)<\int_{0}^{1} H(\eta, s) d \eta .
$$

Proof. If $H(\eta)=H$, then $W_{0}(\xi)=\frac{1}{2}\left(\xi_{1}-H\right)^{2}$, so that the first claim is obvious.

Let the function $H$ be nonconstant; we put $\widehat{W}_{0}(\xi)=W_{0}(\xi)-\frac{1}{2} \xi_{1}^{2}$ and apply the Green formula in the truncated half-strip $\Pi_{R}=\left\{\xi \in \Pi:-R<\xi_{1}\right\}$ :

$$
\begin{aligned}
\frac{1}{2} \int_{\varpi} & \left(\xi_{1}^{2} \partial_{\nu} \Delta_{\xi} W_{0}(\xi)-\partial_{\nu} \xi_{1}^{2} \Delta_{\xi} W_{0}(\xi)\right) d s_{\xi} \\
= & -\int_{\varpi}\left(\widehat{W}_{0}(\xi) \partial_{\nu} \Delta_{\xi} W_{0}(\xi)-\partial_{\nu} \widehat{W}_{0}(\xi) \Delta_{\xi} W_{0}(\xi)\right) d s_{\xi} \\
=\int_{0}^{1}( & W_{0}(\xi) \partial_{1} \Delta_{\xi} \widehat{W}_{0}(\xi)-\partial_{1} W_{0}(\xi) \Delta_{\xi} \widehat{W}_{0}(\xi) \\
& \left.\quad+\Delta_{\xi} W_{0}(\xi) \partial_{1} \widehat{W}_{0}(\xi)-\partial_{1} \Delta_{\xi} W_{0}(\xi) \widehat{W}_{0}(\xi)\right)\left.\right|_{\xi_{1}=-R} d \xi_{2} .
\end{aligned}
$$

Letting $R \rightarrow+\infty$, we replace $W_{0}(\xi)$ and $\widehat{W}_{0}(\xi)$ by the polynomial terms in the corresponding asymptotics (the other terms vanish in the limit). This shows that expression (2.27) is equal to $-P$. On the other hand, the first integral in (2.27) can be written as

$$
\begin{gathered}
-\frac{1}{4} \int_{\varpi} \partial_{\nu} \xi_{1}^{2} \Delta_{\xi} \xi_{1}^{2} d s_{\xi}-\int_{\varpi}\left(\widehat{W}_{0}(\xi) \partial_{\nu} \Delta_{\xi} \widehat{W}_{0}(\xi)-\partial_{\nu} \widehat{W}_{0}(\xi) \Delta_{\xi} \widehat{W}_{0}(\xi)\right) d \xi \\
=-\int_{0}^{1} H\left(\xi_{2}\right) d \xi_{2}+\int_{\Pi}\left|\Delta_{\xi} \widehat{W}_{0}(\xi)\right|^{2} d \xi .
\end{gathered}
$$


This justifies the right inequality in (2.26), because the last-written integral is positive. To check the left inequality in (2.26), we use the function

$$
W_{0}^{\diamond}(\xi)= \begin{cases}W_{0}\left(\xi_{1}\right)-\frac{1}{2}\left(\xi_{1}-H_{0}\right)^{2}, & \xi_{1}<H_{0}, \\ W_{0}\left(\xi_{1}\right), & \xi_{1} \geq H_{0}\end{cases}
$$

here $H_{0}$ is the quantity occurring in (1.16) and (2.26). The function $W_{0}^{\star}$ is still periodic and continuous. Also, it satisfies the homogeneous conditions (2.22) on $\varpi$ and equation (2.21) in the union of the truncated half-strip $\Pi_{0}=\left(-\infty, H_{0}\right) \times(0,1)$ and the set $\Pi^{\star}=$ $\Pi \backslash \bar{\Pi}_{0}$, but the second derivative of $W_{0}^{\star}$ with respect to $\xi_{1}$ has a jump at $\xi_{1}=H_{0}$ (the other derivatives are continuous). We have

$$
\begin{aligned}
\int_{0}^{1} \partial_{1} W_{0}\left(H_{0}, \xi_{2}\right) d \xi_{2}= & \sum_{ \pm} \pm \int_{0}^{1}\left(W_{0}^{\diamond}\left(H_{0} \pm 0, \xi_{2}\right) \partial_{1} \Delta_{\xi} W_{0}^{\diamond}\left(H_{0} \pm 0, \xi_{2}\right)\right. \\
& \left.\quad-\partial_{1} W_{0}^{\diamond}\left(H_{0} \pm 0, \xi_{s}\right) \Delta_{\xi} W_{0}^{\diamond}\left(H_{0} \pm 0, \xi_{2}\right)\right) d \xi_{2} \\
= & -\int_{\Pi_{0}}\left|\Delta_{\xi} W_{0}^{\diamond}(\xi)\right|^{2} d \xi-\int_{\Pi}\left|\Delta_{\xi} W_{0}^{\diamond}(\xi)\right|^{2} d \xi
\end{aligned}
$$

On the other hand, as in (2.27), we find that the first integral in (2.30) is equal to

$$
\begin{aligned}
& \sum_{ \pm} \pm \int_{0}^{1}\left(W_{0}\left(H_{0}, \xi_{2}\right) \partial_{1} \Delta_{\xi} W_{0}^{\diamond}\left(H_{0} \pm 0, \xi_{2}\right)-\partial_{1} W_{0}\left(H_{0}, \xi_{2}\right) \Delta_{\xi} W_{0}^{\bullet}\left(H_{0} \pm 0, \xi_{2}\right)\right. \\
& \left.+\Delta_{\xi} W_{0}\left(H_{0}, \xi_{2}\right) \partial_{1} W_{0}^{\bullet}\left(H_{0} \pm 0, \xi_{2}\right)-\partial_{1} \Delta_{\xi} W_{0}\left(H_{0}, \xi_{2}\right) W_{0}^{\bullet}\left(H_{0} \pm 0, \xi_{2}\right)\right) d \xi_{2} \\
& =\int_{0}^{1}\left(W_{0}(\xi) \partial_{1} \Delta_{\xi} W_{0}^{\star}(\xi)-\partial_{1} W_{0}(\xi) \Delta_{\xi} W_{0}^{\star}(\xi)\right. \\
& \left.+\Delta_{\xi} W_{0}(\xi) \partial_{1} W_{0}^{\diamond}(\xi)-\partial_{1} \Delta_{\xi} W_{0}(\xi) W_{0}^{\diamond}(\xi)\right) d \xi \\
& =\int_{0}^{1} \Delta_{\xi}\left(\frac{1}{2} \xi_{1}^{2}-P \xi_{1}-P_{01}\right) \partial_{1}\left(-P \xi_{1}-P_{01}+H_{0} \xi_{1}-\frac{1}{2} H_{0}^{2}\right) d \xi_{2}=H_{0}-P .
\end{aligned}
$$

This makes the left inequality in (2.26) obvious.

Observe that, by Proposition 2.1, the expression

$$
w_{q}(\xi ; s)=\partial_{n}^{2} u_{q}^{0}(0, s)\left(W_{0}(\xi ; s)-\frac{1}{2} \xi_{1}^{2}+P(s) \xi_{1}+P_{01}(s)\right)
$$

decays exponentially as $\xi_{1} \rightarrow-\infty$, and, therefore, can serve as a boundary layer. We show that this $w_{q}(\xi ; s)$ compensates for the discrepancies in the boundary condition (2.6) left by the sum $u_{q}^{0}(x)+\varepsilon u_{q}^{\prime}(x)$ of the regular type terms in the Ansatz (2.24) (recall that the discrepancy of these terms in the Dirichlet conditions (2.5) is known to be small). By formulas (2.19) and (2.8)-(2.12), the derivative $\partial_{n^{\varepsilon}}$ along the normal to $\Gamma$ differs little from the derivative 3

$$
\varepsilon^{-1} \partial_{\nu}=\varepsilon^{-1}\left(1+\left|\partial_{2} H\left(\xi_{2}, s\right)\right|^{2}\right)^{1 / 2}\left(\partial_{1}-\partial_{2} H\left(\xi_{2}, s\right) \partial_{2}\right)
$$

along the normal to the edge $\varpi(s)$ of the half-strip $\Pi(s)$ (see the definition (2.20) ). Consequently, after freezing the slow variable $s$, the leading part of the discrepancy of the sum $u_{q}^{0}+\varepsilon u_{q}^{\prime}$ in relation (2.6) takes the form

$$
\varepsilon^{-1} \partial_{\nu}\left(\frac{1}{2} n^{2} \partial^{2} u_{q}^{0}(0, s)+\varepsilon n \partial_{n} u_{q}^{\prime}(0, s)\right)=\varepsilon \partial_{\nu}\left(\frac{1}{2} \xi_{1}^{2} \partial_{n}^{2} u_{q}^{0}(0, s)+\xi_{1} \partial_{n} u_{q}^{\prime}(0, s)\right) .
$$

\footnotetext{
${ }^{3}$ The factor $\varepsilon^{-1}$ corresponds to dilation of the coordinates $n$ and $s$.
} 
The identity

$$
\varepsilon^{-1} \partial_{\nu}\left(\varepsilon^{2} w_{q}(\xi ; s)\right)=\varepsilon \partial_{\nu}\left(-\frac{1}{2} \xi_{1}^{2} \partial_{n}^{2} u_{q}^{0}(0, s)+\xi_{1} \partial_{n}^{2} u_{q}^{0}(0, s)\right),
$$

coming from formula (2.25) with the solution $W_{0}$ of the homogeneous problem (2.21)(2.23), shows that it remains to subordinate the correction term $u_{q}^{\prime}$ to the boundary conditions

$$
u_{q}^{\prime}(0, s)=0, \quad \partial_{n} u_{q}^{\prime}(0, s)=-P(s) \partial_{n}^{2} u_{q}^{0}(0, s), \quad s \in \Gamma .
$$

The solvability of problem (2.12), (2.31) is ensured by the $\varkappa_{p}$ relations (2.13), in which the weight factor $H$ is replaced with the factor $P(s)=P_{00}(s)$ (see (2.25)). As a result, the ingredients $a^{q}=\left(a_{1}^{q}, \ldots, a_{\varkappa_{p}}^{q}\right)$ and $\lambda_{q}^{\prime}$ of the asymptotic formulas (2.24), (2.3), and (2.1) can be determined from system (2.14) with the matrix $\mathbf{T}^{q}$ whose entries are given by formulas (2.15) and

$$
h(s)=-P(s) .
$$

This completes the formal asymptotic analysis in the case where $\gamma=1$.

Remark 2.1. We have used the method of compound expansions (see, e.g., 9]); an alternative is the method of matching expansions, which deals with two expansions, the outer expansion

$$
u_{q}^{\varepsilon}(x) \sim u_{q}^{0}(x)+\varepsilon u_{q}^{\prime}(x),
$$

valid far from the perturbed boundary $\Gamma^{\varepsilon}$, and the inner expansion

$$
u_{q}^{\varepsilon}(x) \sim \varepsilon^{\gamma} \mathbf{w}_{q}^{\varepsilon}(x)=\varepsilon^{\gamma} \partial_{n}^{2} u_{q}^{0}(0, s) W_{0}(\xi ; s),
$$

valid in the vicinity of $\Gamma^{\varepsilon}$. The boundary conditions (2.31) arise when we match these expansions. In the next subsection it will be convenient to employ precisely the method of matching expansions.

Remark 2.2. If $\Omega^{\varepsilon} \subset \Omega$ (or $\Omega^{\varepsilon} \supset \Omega$ ), i.e., $H(s, \eta) \leq 0$ (or $H(s, \eta) \geq 0$ ), then, clearly, $\lambda_{j}^{\varepsilon} \geq$ $\lambda_{j}$ (or $\lambda_{j}^{\varepsilon} \leq \lambda_{j}$ ). This observation is in agreement with the asymptotic formula (2.1): the matrix $\mathbf{T}^{p}$ is positive (or negative) and its eigenvalues $\lambda_{p}^{\prime}, \ldots, \lambda_{p+\varkappa_{p}-1}^{\prime}$ are nonnegative (or nonpositive). For a slanting $(\gamma>1)$ perturbation of the boundary the claim is obvious, and for $\gamma=1$ it is ensured by inequalities (2.26). If $\bar{H}(s)=\int_{0}^{1} H(\eta, s) d \eta>0$ (this does not require that the profile function be positive), then the fact that the matrix $\mathbf{T}^{p}$ is nonsingular means that $\lambda_{q}^{\prime}<0$, so that $\lambda_{q}^{\varepsilon}<\lambda_{q}, q=p, \ldots, p+\varkappa_{p}-1$, even if the inclusion $\Omega^{\varepsilon} \subset \Omega$ fails, but only for sufficiently small $\varepsilon>0$.

3. The boundary layer and asymptotic expansions for $\gamma<1$. Assume that, for all $s \in \Gamma$, the function $[0,1) \ni \eta \mapsto H(\eta, s)$ attains its global minimum $H_{0}(s)$ at a unique point $\eta_{0}(s)$, and this is a strict minimum, i.e.,

$$
H(\eta, s)=A(s)\left(\eta-\eta_{0}(s)\right)^{2}+O\left(\left|\eta-\eta_{0}(s)\right|^{3}\right), \quad A(s)>0
$$

(cf. formula (1.16); see also Remarks 2.3 and 3.2 below). We freeze the variable $s=s_{0}$ and introduce the dilated coordinates

$$
\xi=\left(\xi_{1}, \xi_{2}\right)=\left(\varepsilon^{-1} n-\varepsilon^{\gamma-1} H_{0}\left(s_{0}\right), \varepsilon^{-1} s\right) .
$$

They differ from the previous coordinates (2.19) by an additional shift along the first axis. Then the arc $n=\varepsilon^{\gamma} H\left(\varepsilon^{-1} s, s_{0}\right)$ takes the form

$$
\xi_{1}=\varepsilon^{\gamma-1}\left(H\left(s_{0}, \xi_{2}\right)-H_{0}\left(s_{0}\right)\right) .
$$




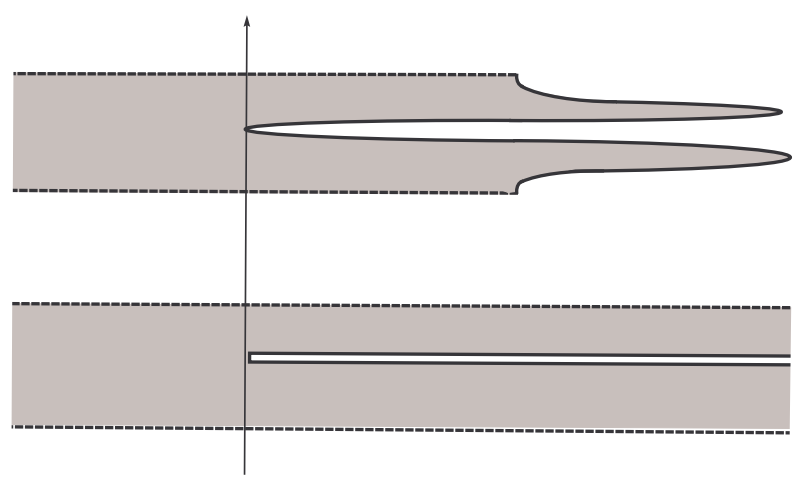

FiguRE 5. Transformation of a strip with strongly curvilinear edge into a strip with a semi-infinite cut.

Since $\gamma-1<0$ and $H\left(\xi_{2}, s_{0}\right)>H_{0}\left(s_{0}\right)$ whenever $\xi_{2} \neq \eta_{0}\left(s_{0}\right)$, a formal passage to the limit as $\varepsilon \rightarrow+0$ transforms the two branches of the arc (2.37) (see Figure 5) into the two shores of the semi-infinite cut $\Upsilon_{ \pm}\left(s_{0}\right)$,

$$
\Upsilon\left(s_{0}\right)=\left\{\xi: \xi_{2}=\eta_{0}\left(s_{0}\right), \xi_{1} \geq 0\right\} .
$$

As a result, for the description of the boundary layer we obtain the domain $\Sigma\left(s_{0}\right)=$ $\Sigma_{0} \backslash \Upsilon\left(s_{0}\right)$, i.e., the strip $\Sigma_{0}=\mathbb{R} \times(0,1)$ with the cut (2.38).

As in Subsection 2 of $\S 2$, we are interested in the $\xi_{2}$-periodic (with period 1) functions $W_{i}$ that satisfy the biharmonic equation in $\Sigma\left(s_{0}\right)$, vanish together with the derivatives on the shores of the cut, and admit expansions (2.25) as $\xi_{1} \rightarrow-\infty$. In other words, we seek special solutions of the homogeneous problem

$$
\begin{aligned}
& \Delta_{\xi}^{2} w(\xi)=0, \quad \xi \in \Sigma, \quad w(\xi)=\partial_{2} w(\xi)=0, \quad \xi \in \Upsilon_{ \pm}, \\
& w\left(\xi_{1}, 0\right)=w\left(\xi_{1}, 1\right), \quad \partial_{2} w\left(\xi_{1}, 0\right)=\partial_{2} w\left(\xi_{1}, 1\right), \quad \xi_{1} \in \mathbb{R} .
\end{aligned}
$$

The existence of the required solutions is also ensured by the results of 44] (see comments on Proposition 2.1). Due to the Dirichlet conditions, the functions $W_{i}$ decay exponentially as $\xi_{1} \rightarrow+\infty$; i.e., for some positive $\delta$ we have

$$
W_{i}(\xi ; s)=O\left(\exp \left(-\delta_{1} \xi_{1}\right)\right), \quad \xi_{1} \rightarrow+\infty, \quad i=0,1 .
$$

The location of the cut is irrelevant, i.e., the quantities $W_{i}\left(\xi_{1}, \xi_{2}-\eta_{0}(s) ; s\right)$ (and, with them, the coefficients $P_{i l}(s)$ ) do not depend on $s$, by periodicity. Repeating with minor changes the final part of the proof of Proposition 2.2 (starting with formula (2.29), where we temporarily put $H_{0}=0$ ), we see that

$$
P=P_{00}=\lim _{\xi \rightarrow-\infty}\left(\partial_{1} W_{0}\left(\xi_{1}, \xi_{2}\right)-\xi_{1}\right)>0 .
$$

In $\S 3$ we shall need the following expansion of the solution $W_{i}(\xi)$ near the vertex of the cut:

$$
W_{i}(\xi)=K_{i} \rho^{3 / 2}\left(3 \cos \frac{\phi}{2}+\cos \frac{3 \phi}{2}\right)+O\left(\rho^{2}\right), \quad \rho \rightarrow+0
$$

(see [51, 46]); here $\rho=|\xi|$ and $\phi \in(-\pi, \pi)$ are the polar coordinates, and $K_{i}$ is some number (the intensity coefficient). Relation (2.42) admits termwise differentiation if we agree that $\nabla_{x} O\left(\rho^{\Lambda}\right)=O\left(\rho^{\Lambda-1}\right)$. 


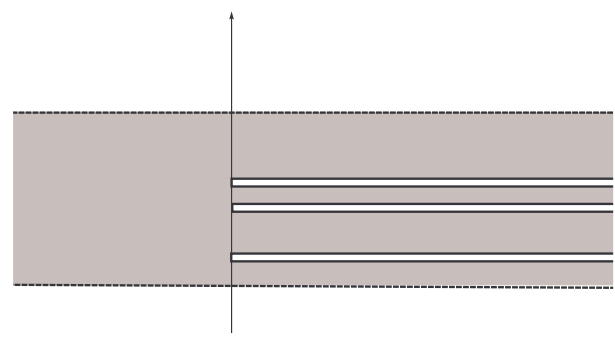

FigURE 6. A strip with several semi-infinite cuts.

Now we proceed to the matching of the outer expansion (2.33) and the inner expansion; as the latter, we take

$$
\begin{aligned}
\varepsilon^{2} \mathbf{w}_{q}^{\varepsilon}(x) & =\partial_{n}^{2} u_{q}^{0}(0, s) W_{0}\left(\varepsilon^{-1}\left(n-\varepsilon^{\gamma} H_{0}(s)\right), \varepsilon^{-1} s-\eta_{0}(s)\right) \\
& =\varepsilon^{2} \partial_{n}^{2} u_{q}^{0}(0, s)\left(\frac{1}{2} \varepsilon^{-2}\left(n-\varepsilon^{-2} H_{0}(s)\right)^{2}-P \varepsilon^{-1}\left(n-\varepsilon^{\gamma} H_{0}(s)\right)-P_{01}+\ldots\right) \\
& =\partial_{n}^{2} u_{q}^{0}(0, s)\left(\frac{1}{2} n^{2}-\varepsilon^{\gamma} n H_{0}(s)+\ldots\right) .
\end{aligned}
$$

Here the dots mean the lower asymptotic terms, which are irrelevant to the formal asymptotic analysis in question. The same leading terms appear also in the outer expansion

$$
u_{q}^{0}(x)+\varepsilon^{\gamma} u_{q}^{\prime}(x)=\frac{1}{2} n^{2} \partial_{n}^{2} u_{q}^{0}(0, s)+\varepsilon^{\gamma} n \partial_{n} u_{q}^{\prime}(0, s)+O\left(n^{2}\left(n+\varepsilon^{\gamma}\right)\right),
$$

provided that the correction $u_{q}^{\prime}$ satisfies the boundary conditions (2.11) with the replacement $H(s) \mapsto H_{0}(s)$.

All subsequent arguments that lead to system (2.14) and the matrix $\mathbf{T}^{p}$ with the entries (2.15), where

$$
h(s)=H_{0}(s),
$$

need no modification. Note that the resulting formula for the eigenvalues becomes meaningful only if the function $H_{0}$ is not equal to 0 identically on $\Gamma$.

4. The case where $\gamma<1, H_{0} \not \equiv 0$. Assume identity (1.16). Then relation (2.43) takes the form

$$
\varepsilon^{2} \mathbf{w}_{q}^{\varepsilon}(x)=\partial_{n}^{2} u_{q}^{0}(0, s)\left(\frac{1}{2} n^{2}-P \varepsilon n+\cdots\right),
$$

and the procedure of matching with the outer expansion (2.33) leads to the boundary conditions (2.31) with the constant factor (2.41), and also allows us to repeat what was said after formula (2.41), applying the arguments to the case under consideration.

Remark 2.3. If the profile function $[0,1) \ni \eta \mapsto H(s, \eta)$ attains its minimal value at several points $\eta_{0}(s), \ldots, \eta_{N}(s)$, and the number $N$ stays constant for all $s \in \Gamma$, then our asymptotic construction needs no essential modification: in this case the role of the domain $\Sigma(s)$ is played by a strip with several half-infinite cuts whose ends have one and the same abscissa (see Figure 6 , where $N=3$ ). Difficulties arise if at least two points merge. The authors do not know the structure of the asymptotics in question in the latter case. 


\section{§3. JUSTIFICATION OF ASYMPTOTIC FORMULAS}

FOR PERIODIC PERTURBATION OF THE BOUNDARY

1. Abstract formulation of the problem. We denote by $H^{\varepsilon}$ the Hilbert space $\stackrel{\circ}{H}^{2}\left(\Omega^{\varepsilon}\right)$ with the scalar product

$$
\left\langle u^{\varepsilon}, v^{\varepsilon}\right\rangle_{\varepsilon}=\left(\Delta_{x} u^{\varepsilon}, \Delta_{x} v^{\varepsilon}\right)_{\Omega^{\varepsilon}} .
$$

On that space, we introduce the operator $K^{\varepsilon}$ by the formula

$$
\left\langle K^{\varepsilon} u^{\varepsilon}, v^{\varepsilon}\right\rangle_{\varepsilon}=\left(u^{\varepsilon}, v^{\varepsilon}\right)_{\Omega^{\varepsilon}}, \quad u^{\varepsilon}, v^{\varepsilon} \in H^{\varepsilon} .
$$

This operator is continuous and symmetric; hence, it is selfadjoint. Also, it is positive and compact. The variational problem (1.5) is equivalent to the abstract equation

$$
K^{\varepsilon} u^{\varepsilon}=\mu^{\varepsilon} u^{\varepsilon} \quad \text { in } \quad H^{\varepsilon}
$$

with the new spectral parameter

$$
\mu^{\varepsilon}=1 / \lambda^{\varepsilon} .
$$

By [2, Theorems 10.1.5 and 10.2.2], the spectrum of $K^{\varepsilon}$ consists of the essential spectrum $\{\mu=0\}$ and the discrete spectrum forming a positive infinitesimally small sequence of eigenvalues

$$
\mu_{1}^{\varepsilon} \geq \mu_{2}^{\varepsilon} \geq \cdots \geq \mu_{p}^{\varepsilon} \geq \cdots \rightarrow+0 .
$$

Now, as eigenvectors we take $U_{p}^{\varepsilon}=\left(\lambda_{p}^{\varepsilon}\right)^{-1 / 2} u_{p}^{\varepsilon}$; they obey the natural orthogonality and normalization condition

$$
\left\langle U_{p}^{\varepsilon}, U_{q}^{\varepsilon}\right\rangle_{\varepsilon}=\delta_{p, q}, \quad p, q \in \mathbb{N} .
$$

The next statement is known as the lemma about "near eigenvalues and eigenvectors" (see [52], and also [2, Chapter 6]).

Lemma 3.1. Suppose $\mathcal{U}^{\varepsilon} \in H^{\varepsilon},\left\|\mathcal{U}^{\varepsilon} ; H^{\varepsilon}\right\|=1, \mathcal{M}^{\varepsilon}>0$, and

$$
\delta^{\varepsilon}=\left\|K^{\varepsilon} \mathcal{U}^{\varepsilon}-\mathcal{M}^{\varepsilon} \mathcal{U}^{\varepsilon} ; H^{\varepsilon}\right\|<\mathcal{M}^{\varepsilon} .
$$

Then the segment $\left[\mathcal{M}^{\varepsilon}-\delta^{\varepsilon}, \mathcal{M}^{\varepsilon}+\delta^{\varepsilon}\right]$ contains at least one eigenvalue of the operator $K^{\varepsilon}$. Moreover, for any $\Delta^{\varepsilon} \in\left(\delta^{\varepsilon}, \mathcal{M}^{\varepsilon}\right)$, there exist coefficients $A_{j}^{\varepsilon}$ such that

$$
\left\|\mathcal{U}^{\varepsilon}-\sum_{j=P^{\varepsilon}}^{P^{\varepsilon}+X^{\varepsilon}-1} A_{j}^{\varepsilon} U_{j}^{\varepsilon} ; H^{\varepsilon}\right\| \leq 2 \frac{\delta^{\varepsilon}}{\Delta^{\varepsilon}}, \quad \sum_{j=P^{\varepsilon}}^{P^{\varepsilon}+X^{\varepsilon}-1}\left|A_{j}^{\varepsilon}\right|^{2}=1,
$$

where it is assumed that $\mu_{P^{\varepsilon}}^{\varepsilon}, \ldots, \mu_{P^{\varepsilon}+X^{\varepsilon}-1}^{\varepsilon}$ form the complete list of the elements in (3.5) that fall into the segment $\left[\mathcal{M}^{\varepsilon}-\Delta^{\varepsilon}, \mathcal{M}^{\varepsilon}+\Delta^{\varepsilon}\right]$.

2. Plan of the proof and statement of the theorem on asymptotic formulas. In the next subsection we shall choose approximate "eigenvalues"

$$
\mathcal{M}_{q}^{\varepsilon}=\left(\lambda_{p}+\varepsilon^{\gamma_{0}} \lambda_{q}^{\prime}\right)^{-1}, \quad q=p, \ldots, p+\varkappa_{p}-1,
$$

and the corresponding approximate "eigenfunctions"

$$
\mathcal{U}_{p}^{\varepsilon}, \ldots, \mathcal{U}_{p+\varkappa_{p}-1}^{\varepsilon}
$$

of the operator $K^{\varepsilon}$. Then we shall show that the resulting quantities (3.7) satisfy

$$
\delta_{q}^{\varepsilon} \leq c_{p} \varepsilon^{\gamma_{1}} .
$$


The exponents in (3.9) and (3.10) are determined as follows :

$$
\gamma_{0}=\left\{\begin{array}{ll}
\gamma & \text { if } \gamma \geq 1, \\
\gamma & \text { if } \gamma<1, \quad H_{0} \equiv 0, \\
1 & \text { if } \gamma<1, \quad H_{0} \equiv 0,
\end{array} \quad \gamma_{1}= \begin{cases}2 \gamma-1 / 2 & \text { if } \gamma<1, \\
3 / 2 & \text { if } \gamma=1, \\
(1+\gamma) / 2 & \text { if } \gamma<1, \quad H_{0} \neq 0 \\
1+\gamma / 2 & \text { if } \gamma<1, \quad H_{0} \equiv 0\end{cases}\right.
$$

Next, from estimate (3.10) and Lemma 3.1 we shall deduce that for each $p$ (see (1.17)) in the sequence (3.5) we can find $\varkappa_{p}$ distinct elements $\mu_{j(p)}^{\varepsilon}, \ldots, \mu_{j\left(p+\varkappa_{p}-1\right)}^{\varepsilon}$ such that

$$
\left|\mu_{j(q)}^{\varepsilon}-\mathcal{M}_{q}^{\varepsilon}\right| \leq c_{p} \varepsilon^{\gamma_{1}} \quad \Rightarrow \quad\left|\lambda_{j(q)}^{\varepsilon}-\lambda_{p}-\varepsilon^{\gamma_{0}} \lambda_{q}^{\prime}\right| \leq C_{p} \varepsilon^{\gamma_{1}}
$$

for $\varepsilon \in\left(0, \varepsilon_{p}\right]$; here $\varepsilon_{p}>0$ and $q=p, \ldots, p+\varkappa_{p}-1$. The implication (3.12) is ensured by the following simple observation:

$$
\left|\frac{1}{a}-\frac{1}{b}\right| \leq c \epsilon \quad \Rightarrow \quad\left\{\begin{array}{l}
|a-b| \leq c a b \epsilon \\
a \leq(1-c b \epsilon)^{-1} b
\end{array} \quad \Rightarrow \quad|a-b| \leq \frac{c b^{2}}{1-c b \epsilon} \epsilon .\right.
$$

Thus, it remains to check that in (3.12) we have $j(q)=q$. This is done with the help of Lemma 1.1, which we are now going to prove. First, we note that, by the minimax principle (see [2, Theorem 10.2.2]), we have $\lambda_{q}^{\varepsilon} \leq \lambda_{q}^{\nabla}, q \in \mathbb{N}$, where the $\lambda_{q}^{\nabla}$ are the eigenvalues of problem (1.11) in a fixed domain $\Omega^{\nabla}$ included in $\Omega^{\varepsilon}$ for any $\varepsilon \in\left(0, \varepsilon_{0}\right]$; here $\varepsilon_{0}>0$. Thus, $\lambda_{q}^{\varepsilon_{(m)}} \rightarrow \lambda_{q}^{0}$ as $m \rightarrow+\infty$, along a certain infinitesimally small sequence $\left\{\varepsilon_{(m)}\right\}$. Extending the eigenfunctions $u_{q}^{\varepsilon}$ to a domain $\Omega^{\Delta}$ containing $\Omega^{\varepsilon}$ for any $\varepsilon \in\left(0, \varepsilon^{\Delta}\right]$, we see that the norms $\left\|u_{q}^{\varepsilon} ; H^{2}\left(\Omega^{\Delta}\right)\right\|$ are bounded, because of inequality (1.6), the normalization conditions (1.8), and the formula $\left.\left\|\Delta_{x} u_{q}^{\varepsilon} ; L_{2}\left(\Omega^{\varepsilon}\right)\right\|^{2}=\lambda_{q}^{\varepsilon} \| u_{q}^{\varepsilon} ; L_{2} \Omega^{\varepsilon}\right) \|^{2}$, implied by (1.5). As a result, rarefying the sequence $\left\{\varepsilon_{(m)}\right\}$ if necessary, we see that

$$
u_{q}^{\varepsilon_{(m)}} \rightarrow u_{q}^{0} \text { weakly in } \stackrel{\circ}{H}^{2}\left(\Omega^{\Delta}\right) \text { and strongly in } L_{2}\left(\Omega^{\Delta}\right),
$$

and that $\left\|u_{q}^{0} ; L_{2}\left(\Omega^{\Delta}\right)\right\|=1$. Since the supports of $u_{q}^{\varepsilon_{(m)}}$ contract to the set $\Omega$, we have $u_{q}^{0} \in \stackrel{\circ}{H}^{2}(\Omega)$.

Finally, we fix some smooth function $v$ with compact support in $\Omega$. By (1.1), this function lies in $\stackrel{\circ}{H}^{2}\left(\Omega^{\varepsilon}\right)$ for sufficiently small $\varepsilon$. Plugging $v$ into (1.5) and passing to the limit, we see that the limits $\lambda_{q}^{0}$ and $u_{q}^{0}$ satisfy (1.11). Now, Lemma 1.1 became obvious, because $u_{q}^{0} \neq 0$.

We turn to the identities $j(q)=q$; it suffices to show that $j\left(p+\varkappa_{p}-1\right)=p+\varkappa_{p}-1$. The latter number will be denoted by $P$. The relation $j(P)<P$ is impossible because for each eigenvalue $\lambda_{k}$ with $k \in\{1, \ldots, P\}$ we have an eigenvalue $\lambda_{j(k)}^{\varepsilon}$ of problem (1.5) and $j(k) \neq j(m)$ whenever $k \neq m$. If $j(m)>m$, then there is an eigenfunction $u_{P(\varepsilon)}^{\varepsilon}$ that corresponds to an eigenvalue $\lambda_{P(\varepsilon)}^{\varepsilon} \leq \lambda_{P}+c_{p} \varepsilon^{\gamma_{0}}$ and is orthogonal in $L_{2}\left(\Omega^{\varepsilon}\right)$ to each of the eigenfunctions $u_{j(1)}^{\varepsilon}, \ldots, u_{j(P)}^{\varepsilon}$. Repeating the proof of Lemma 1.1 and observing that the orthogonality conditions survive after the limit passage as $\varepsilon_{(m)} \rightarrow 0$, we see the following: the dimension of the subspace corresponding to the eigenvalues of the limiting problem (1.11) that lie on the segment $\left[0, \lambda_{P}\right]$ is strictly greater than $P$, a contradiction.

Now we state a result to be proved in what follows.

Theorem 3.1. 1) Let $\lambda_{p}$ be an eigenvalue of multiplicity $\varkappa_{p}$ for the limiting problem (1.9), (1.10) (or (1.11) in the variational setting), and let $u_{p}, \ldots, u_{p+\varkappa_{p}-1}$ be the corresponding eigenfunctions subject to the orthogonality and normalization conditions (2.4). There exist positive numbers $\varepsilon_{p}$ and $c_{p}, C_{p}$ such that, for $\varepsilon \in\left(0, \varepsilon_{p}\right]$, the eigenvalues 
$\lambda_{p}^{\varepsilon}, \ldots, \lambda_{p+\varkappa_{p}-1}^{\varepsilon}$ of the singularly perturbed problem (1.3), (1.4) (or (1.5) in the variational setting) satisfy the inequalities

$$
\left|\lambda_{q}^{\varepsilon}-\lambda_{p}-\varepsilon^{\gamma_{0}} \lambda_{q}^{\prime}\right| \leq c_{p} \varepsilon^{\gamma_{1}}, \quad q=p, \ldots, p+\varkappa_{p}-1
$$

where $\gamma_{0}$ and $\gamma_{1}$ are the exponents (3.11), $\lambda_{p}^{\prime}, \ldots, \lambda_{p+\varkappa_{p}-1}^{\prime}$ are the eigenvalues (2.16) of the matrix $\mathbf{T}$ of size $\varkappa_{p} \times \varkappa_{p}$ with the entries defined by formula (2.15) with

$$
T(s)= \begin{cases}\bar{H}(s) & \text { if } \gamma>1 \\ P(s) & \text { if } \gamma=1\end{cases}
$$

and for $\gamma<1$,

$$
T(s)= \begin{cases}H_{0}(s) & \text { if } H_{0} \neq 0 \text { on } \Gamma \\ P & \text { if } H_{0} \equiv 0 \text { on } \Gamma .\end{cases}
$$

Here $\bar{H}$ is the mean value (1.15), $H_{0}$ is the minimal value (see (1.16)) of the profile function $H$ that describes the rapidly oscillating boundary (1.1), and $P(s)$ and $P$ are the coefficients in the expansions (2.25) of the special solutions $W_{0}$ of the homogeneous problem (2.21)-(2.23) in the half-strip (2.20) with a curvilinear edge and of problem (2.39) in the unit strip $\Sigma_{0}$ with the cut (2.38).

2) There are coefficient columns $b_{q}^{\varepsilon}=\left(b_{q, p}^{\varepsilon}, \ldots, b_{q, p+\varkappa_{p}-1}^{\varepsilon}\right)$ forming an orthogonal $\left(\varkappa_{p} \times \varkappa_{p}\right)$-matrix such that for the eigenfunctions $u_{p}^{\varepsilon}, \ldots, u_{p+\varkappa_{p}-1}^{\varepsilon}$ of problem (1.5), orthonormalized as in (1.8), we have the asymptotic formulas

$$
\left\|u_{q}^{\varepsilon}-\sum_{j=p}^{p+\varkappa_{p}-1} b_{q, j}^{\varepsilon} u_{j} ; H^{2}\left(\Omega^{\varepsilon}\right)\right\| \leq C_{p} \varepsilon^{\gamma / 2} .
$$

The eigenfunctions $u_{p}, \ldots, u_{p+\varkappa_{p}-1}$ of problem (1.11) obey the orthogonality and normalization conditions (2.4) and are extended by zero outside the limiting domain $\Omega$.

3. Global asymptotic approximation. Suppose $\gamma \leq 1$ (the case where $\gamma>1$ is much simpler and will be considered in passing in Subsection 6 of $\S 3$ ). We operate with the eigenvectors $a^{p}, \ldots, a^{p+\varkappa_{p}-1}$ of the matrix $\mathbf{T}^{p}$ to form $\varkappa_{p}$ global asymptotic approximations to the eigenfunctions of problem (1.3), (1.4):

$$
\begin{aligned}
\mathbf{U}_{q}^{\varepsilon}(x)= & \mathcal{X}_{\varepsilon}(x)\left(u_{q}^{0}(x)+\varepsilon^{\gamma_{0}} u_{q}^{\prime}(x)\right)+\chi(x) \varepsilon^{2} \partial_{n}^{2} u_{q}^{0}(0, s) W_{0}(\xi ; s) \\
& -\mathcal{X}_{\varepsilon}(x) \chi(x)\left(\frac{1}{2} n^{2} \partial_{n}^{2} u_{q}^{0}(0, s)+\varepsilon^{\gamma_{0}} n \partial_{n} u_{q}^{\prime}(0, s)\right)+\mathcal{W}_{q}^{\varepsilon}(x) .
\end{aligned}
$$

This formula needs an explanation. By $u_{q}^{0}$ we mean the linear combination (2.3), and $u_{q}^{\prime}$ is the solution of the differential equation of (2.12) with the boundary conditions (2.11) or 2.31) in which the weight factor of $\partial_{n}^{2} u_{q}^{0}(0, s)$ is chosen in accordance with the asymptotic constructions of Subsections 4 and 5 of $\S 2$ (see (3.14) and (3.15)), and solvability is ensured by conditions of the form (2.13). The cutoff functions $\mathcal{X}_{\varepsilon}$ and $\chi$ are defined by the formulas

$$
\chi(x)=\left\{\begin{array}{ll}
\chi_{0}(n), & x \in \mathcal{V}, \\
0, & x \in \Omega^{\varepsilon} \backslash \mathcal{V},
\end{array} \quad \mathcal{X}_{\varepsilon}(x)= \begin{cases}1-\chi_{0}\left(\varepsilon^{-\gamma_{0}} n\right), & x \in \mathcal{V}, \\
1, & x \in \Omega^{\varepsilon} \backslash \mathcal{V},\end{cases}\right.
$$

where $\chi_{0} \in C^{\infty}(\mathbb{R}), \chi_{0}(n)=1$ if $|n|<d / 2, \chi_{0}(n)=0$ if $|n|>d$, and $0 \leq \chi \leq 1$; the parameter $d$ is chosen so that the functions (3.17) are smooth and $\mathcal{X}_{\varepsilon}=0$ on $\Gamma^{\varepsilon}$. The boundary layer involves the special solution $W_{0}$ of the homogeneous problem (2.21) (2.23) for $\gamma=1$ and of problem (2.39) for $\gamma<1$. The dilated coordinates $\xi$ are given by (2.19) for $\gamma_{0}=1$ and by (2.36) for $\gamma<1, H_{0} \not \equiv 0$. Finally, $\mathcal{W}_{q}^{\varepsilon}$ is an additional term ensuring that $U_{q}^{\varepsilon} \in \stackrel{\circ}{H}^{2}(\Omega)$, i.e., compensating for the small discrepancy in the 
boundary condition (2.6) for the third summand (see explanations below), and subject to the estimate

$$
\left\|\mathcal{W}_{q}^{\varepsilon} ; \stackrel{\circ}{H}\left(\Omega^{\varepsilon}\right)\right\| \leq c \varepsilon^{\gamma_{2}}, \quad \gamma_{2}= \begin{cases}2 \gamma-3 / 2 & \text { if } \gamma>1, \\ 3 / 2 & \text { if } \gamma=1, \\ (\gamma+1) / 2 & \text { if } \gamma<1, H_{0} \neq 0, \\ 1 & \text { if } \gamma<1, H_{0} \equiv 0 .\end{cases}
$$

It should be noted that we employ an asymptotic construction with "overlapping" cutoff functions (3.17) (see [53] and also [9, Chapter 2]): in the third summand on the right in (3.16) we collected all terms that have undergone the matching procedure (cf. Remark 2.1). These terms occur in the first and the second summand, but duplication is eliminated by the subtrahend. When commuting the Laplace operator with cutoff functions (we need this for estimating the remainder terms), we shall append this subtrahend to one or the other summand, in order that the differences decay in the zone under consideration. We illustrate the corresponding calculations.

The definition (3.17) of our cutoff functions (more precisely, the location of their supports) shows that

$$
\chi\left[\Delta_{x}, \mathcal{X}_{\varepsilon}\right]=\left[\Delta_{x}, \mathcal{X}_{\varepsilon}\right], \quad \mathcal{X}_{\varepsilon}\left[\Delta_{x}, \chi\right]=\left[\Delta_{x}, \chi\right]
$$

here $\left[\Delta_{x}, \chi\right] v=2 \nabla_{x} v \cdot \nabla_{x} \chi+v \Delta_{x} \chi$. Now, we write the right-hand side of (3.16) without the last term $\mathcal{W}_{q}^{\varepsilon}$ as the algebraic sum

$$
\mathcal{X}_{\varepsilon} S_{o}^{\varepsilon}+\chi S_{i}^{\varepsilon}-\mathcal{X}_{\varepsilon} \chi S_{m}^{\varepsilon},
$$

and perform commutation accompanied with a rearrangement of terms as indicated above. As a result, we get

$$
\begin{aligned}
\Delta_{x}\left(\mathcal{X}_{\varepsilon} S_{o}^{\varepsilon}+\chi S_{i}^{\varepsilon}-\mathcal{X}_{\varepsilon} \chi S_{m}^{\varepsilon}\right)= & \mathcal{X}_{\varepsilon} \Delta_{x} S_{o}^{\varepsilon}+\chi \Delta_{x} S_{i}^{\varepsilon}+\chi \Delta_{x} S_{i}^{\varepsilon}-\mathcal{X}_{\varepsilon} \chi S_{m}^{\varepsilon} \\
& +\left[\Delta_{x}, \mathcal{X}_{\varepsilon}\right]\left(S_{o}^{\varepsilon}-S_{m}^{\varepsilon}\right)+\left[\Delta_{x}, \chi\right]\left(S_{i}^{\varepsilon}-S_{m}^{\varepsilon}\right) .
\end{aligned}
$$

Since $\left|\nabla_{x}^{k} \mathcal{X}_{\varepsilon}(x)\right| \leq c_{k} \varepsilon^{-k \gamma_{0}}$ and the coefficients of the differential operator $\left[\Delta_{x}, \mathcal{X}_{\varepsilon}\right]$ vanish for $|n| \geq c \varepsilon^{\gamma_{0}}$, we see that

$$
\begin{aligned}
& \left|\left[\Delta_{x}, \mathcal{X}_{\varepsilon}\right]\left(S_{o}^{\varepsilon}(x)-S_{m}^{\varepsilon}(x)\right)\right| \\
& \quad=\mid\left[\Delta_{x}, \mathcal{X}_{\varepsilon}\right]\left(u_{q}^{0}(x)-\frac{1}{2} n^{2} \partial_{n}^{2} u_{q}^{0}(0, s)+\varepsilon^{\gamma_{0}}\left(u_{q}^{\prime}(x)-n \partial_{n} u_{q}^{\prime}(0, s)\right) \mid\right. \\
& \quad \leq c\left(\varepsilon^{-\gamma_{0}}\left(n^{2}+\varepsilon^{-\gamma_{0}}|n|\right)+\varepsilon^{-2 \gamma_{0}}\left(|n|^{3}+\varepsilon^{\gamma_{0}} n^{2}\right)\right) \leq c \varepsilon^{\gamma_{0}} .
\end{aligned}
$$

Consider the last term in (3.20). First, let $\gamma_{0}=1$, i.e., either $\gamma=1$, or $\gamma<1$, but $H_{0} \equiv 0$. Then (2.25) and the second boundary condition in (2.31) imply that

$$
S_{i}^{\varepsilon}(x)-S_{m}^{\varepsilon}(x)=\varepsilon^{2} \partial_{n}^{2} u_{q}^{0}(0, s)\left(\widetilde{W}_{0}\left(\varepsilon^{-1} n, \varepsilon^{-1} s ; s\right)-P_{01}(s)\right),
$$

and the quantity

$$
\widetilde{W}_{0}(\xi ; s)=W_{0}(\xi ; s)-\frac{1}{2} \xi_{1}^{2}+P(s) \xi_{1}+P_{01}(s)
$$

decays exponentially as $\xi_{1} \rightarrow-\infty$; here the free term $P_{01}(s)$ in the first expansion in $(2.25)$ is a smooth function of $s \in \Gamma$. Thus, expression (3.22) is written as the sum of two terms: a smooth function multiplied by $\varepsilon^{2}$ and a quantity that is exponentially small on the set $\operatorname{supp}\left|\nabla_{x} \chi\right|$, i.e., on the support of the coefficients of the commutator $\left[\Delta_{x}, \chi\right]$, where $\xi_{1}=\varepsilon^{-1} n<c_{\chi} \varepsilon^{-1}, c_{\chi}>0$. Therefore,

$$
\begin{aligned}
\left\|\left[\Delta_{x}, \mathcal{X}_{\varepsilon}\right]\left(S_{o}^{\varepsilon}-S_{m}^{\varepsilon}\right) ; L_{2}\left(\Omega^{\varepsilon}\right)\right\|^{2} & \leq c \varepsilon^{2 \gamma_{0}} \varepsilon^{\gamma_{0}}, \\
\left\|\left[\Delta_{x}, \chi\right]\left(S_{i}^{\varepsilon}-S_{m}^{\varepsilon}\right) ; L_{2}\left(\Omega^{\varepsilon}\right)\right\|^{2} & \leq c\left(\varepsilon^{2}+\exp \left(-\delta_{\chi} \varepsilon^{-1}\right)\right), \quad \delta_{\chi}>0 .
\end{aligned}
$$


In the first line in (3.24), one of the factors $\varepsilon^{\gamma_{0}}$ arose because of integration over the boundary strip of width $O\left(\varepsilon^{\gamma_{0}}\right)$ on which the support of the function on the left in (3.21) is located. For similar reasons,

$$
\left\|\chi \Delta_{x} S_{i}^{\varepsilon}-\mathcal{X}_{\varepsilon} \chi \Delta_{x} S_{m}^{\varepsilon} ; L_{2}\left(\Omega^{\varepsilon}\right)\right\|^{2} \leq c \varepsilon^{\gamma_{0}} .
$$

Indeed, the factor $\varepsilon^{2}$ of the second term on the right in (3.16) suppresses the large factors $\varepsilon^{-2}$ and $\varepsilon^{-1}$ coming from differentiation (at most two-fold) with respect to the fast variables (2.19), and the expression on the left in (3.25) without the smooth subtrahend $P_{01}(s)$, for which the required estimate is obvious, decays exponentially as $\xi_{1}=\varepsilon^{-1} n \rightarrow$ $-\infty$. This means that, in essence, in (3.25) it remains to consider the integral

$$
C \int_{\Gamma} \int_{-c_{-}}^{c_{+} \varepsilon} \exp \left(2 \delta \varepsilon^{-1} n\right) d n d s
$$

which is $O\left(\varepsilon^{\gamma_{0}}\right)$ (recall that $\gamma_{0}=1$ ). Finally, $\operatorname{supp}\left(\mathcal{X}_{\varepsilon} \Delta_{x} S_{0}^{\varepsilon}\right) \subset \Omega$ and

$$
\left\|\mathcal{X}_{\varepsilon} \Delta_{x} S_{o}^{\varepsilon}-\Delta_{x} u_{q}^{0} ; L_{2}(\Omega)\right\|^{2} \leq c \varepsilon^{\gamma_{0}} .
$$

Formulas (3.24)-(3.26) and (3.18) show that

$$
\left|\left(\Delta_{x} \mathbf{U}_{q}^{\varepsilon}, \Delta_{x} \mathbf{U}_{l}^{\varepsilon}\right)_{\Omega^{\varepsilon}}-\left(\Delta_{x} u_{q}^{0}, \Delta_{x} u_{l}^{0}\right)_{\Omega}\right| \leq c \varepsilon^{\gamma_{0} / 2} .
$$

In particular, using (2.4) and (1.11), we obtain

$$
1-c \varepsilon^{\gamma_{0} / 2} \leq\left\|\mathbf{U}_{q}^{\varepsilon} ; H^{\varepsilon}\right\|-\lambda_{q} \leq 1+c \varepsilon^{\gamma_{0} / 2} .
$$

If $\gamma_{0}<1$, i.e., $\gamma<1$, but $H_{0} \not \equiv 0$, then the calculations remain largely the same. The only point that is worth attention is a modification of the last factor in formulas similar to (3.22) and (3.23):

$$
\begin{aligned}
S_{i}^{\varepsilon}(x)-S_{m}^{\varepsilon}(x) & =\varepsilon^{2} \partial_{n}^{2} u_{q}^{0}(0, s) W_{0}^{\bullet}\left(\varepsilon^{-1} n-\varepsilon^{\varepsilon-1} H_{0}(s), \varepsilon^{-1} s ; s\right), \\
W_{0}^{\bullet}(\xi ; s)= & W_{0}(\xi ; s)-\varepsilon^{-2} \frac{1}{2} n^{2}-\varepsilon^{-2+\gamma} H_{0}(s) n \\
= & W_{0}(\xi ; s)-\frac{1}{2} \xi_{1}^{2}-\frac{1}{2} \varepsilon^{2 \gamma-2} H_{0}(s)^{2} \widetilde{W}_{0}(\xi, s) \\
& \quad-P(s) \xi_{1}-P_{01}(s)-\frac{1}{2} \varepsilon^{2 \gamma-2} H_{0}(s)^{2} .
\end{aligned}
$$

We have used the definition (2.36) of the fast variable $\xi_{1}$. The linear growth of $W_{0}^{\bullet}(\xi, s)$ does not impede the deduction of estimates, though it enlarges the majorant, which, however, is irrelevant because $\gamma<1$. Indeed, for two-fold differentiation the estimate survives because of the presence of $\varepsilon^{2}$ and the exponential decay of $\widetilde{W}_{0}$, while for one-fold differentiation or for differentiation only in the slow variable $s$, a similar role is played by the remaining factors $\varepsilon$ or $\varepsilon^{2}$, respectively. The norm in (3.25) can be treated similarly.

Thus, formulas (3.27) and (3.28) are valid also for $\gamma_{0}=\gamma<1$.

4. Discrepancy estimate for $\gamma=1$. First, we find the correction term $\mathcal{W}_{q}^{\varepsilon}$ in (3.16). Due to the cutoff function $\mathcal{X}_{\varepsilon}$, the first and third terms on the right in (3.16) satisfy the boundary conditions (1.4). Since $W^{0}$ is a solution of the homogeneous problem (2.21)-(2.23),$W^{0}$ also satisfies the first boundary condition, so that we should put

$$
\mathcal{W}_{q}^{\varepsilon}(x)=0, \quad s \in \Gamma^{\varepsilon} .
$$

On the other hand, the derivative along the normal to the boundary (1.1),

$$
\begin{aligned}
& \partial_{n^{\varepsilon}}\left(\partial_{n}^{2} u_{q}^{0}(0, s) W_{0}\left(\varepsilon^{-1} n, \varepsilon^{-1} s, s\right)\right) \\
= & \left.\left(1+N^{\varepsilon}(s)^{2}\right)^{-1 / 2} \frac{1}{\varepsilon}\left(\frac{\partial}{\partial \xi_{1}}-N^{\varepsilon}(s) J^{\varepsilon}(s)^{-1}\left(\varepsilon \frac{\partial}{\partial s}+\frac{\partial}{\partial \xi_{2}}\right)\right)\left(\partial_{n}^{2} u_{q}^{0}(0, s) W_{0}(\xi, s)\right)\right|_{\xi=\varepsilon^{-1}(n, s)},
\end{aligned}
$$




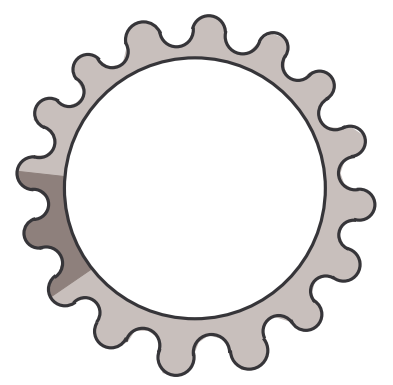

FiguRE 7. Periodic boundary layer and a cell of the covering.

found by formulas (2.7) and (2.8), differs from the derivative along the normal to the edge $\varpi(s)$ of the half-strip $\Pi(s)$ (see $(2.20)$ ) for $s$ fixed:

$$
\left.\frac{1}{\varepsilon}\left(1+\left|\frac{\partial H}{\partial \xi_{2}}\left(\xi_{s}, s\right)\right|^{2}\right)^{-1 / 2} \frac{1}{\varepsilon}\left(\frac{\partial}{\partial \xi_{1}}-\frac{\partial H}{\partial \xi_{2}}\left(\xi_{2}, s\right) \frac{\partial}{\partial \xi_{2}}\right)\left(\partial_{n}^{2} u_{q}^{0}(0, s) W_{0}(\xi, s)\right)\right|_{\xi=\varepsilon^{-1}(n, s)} .
$$

Formulas (2.9) and (2.10) show that the difference is $O\left(\varepsilon^{0}\right)$. As a result, taking the factor $\varepsilon^{2}$ into account, we obtain

$$
\partial_{n^{\varepsilon}} \mathcal{W}_{q}^{\varepsilon}(x)=\varepsilon^{2} \mathcal{P}_{1}\left(\varepsilon, \varepsilon^{-1} s, s\right), \quad x=s \in \Gamma .
$$

Here $\mathcal{P}_{1}$ is a smooth function of the variables $\varepsilon \in[0,1], \eta=\varepsilon^{-1} s \in[0,1]$, and $s \in \Gamma$, periodic relative to $\eta$.

The set

$$
\Theta^{\varepsilon}=\left\{x \in \Omega^{\varepsilon}: n>\varepsilon\left(1+\min \left\{H_{0}(\eta, s) \mid \eta \in[0,1], s \in \Gamma\right\}\right)\right\}
$$

(it is shadowed in Figure 7; cf. Figure 1) can be covered by the cells

$$
\theta_{j}^{\varepsilon}=\left\{x \in \Theta^{\varepsilon}: s \in(\varepsilon j-\varepsilon / 2, \varepsilon j+3 \varepsilon / 2)\right\}, \quad j=1, \ldots, N
$$

(one of these cells is deeply shadowed in Figure 7). We recall that $\varepsilon=1 / N$. Each of the cells "differs little" from the other cells (one is taken to another by a smooth diffeomorphism). We choose an appropriate partition of unity $\left\{\zeta_{j}^{\varepsilon}\right\}_{j=1}^{N}$ on the contour $\Gamma$, with elements $\zeta_{j}^{\varepsilon} \in C_{c}^{\infty}(\varepsilon j-\varepsilon / 4, \varepsilon j+5 \varepsilon / 4)$. We construct functions $\mathcal{W}_{q j}^{\epsilon} \in C_{c}^{\infty}\left(\theta_{j}^{\varepsilon} \cup \vartheta_{j}^{\varepsilon}\right)$ such that

$$
\mathcal{W}_{q j}^{\varepsilon}=0, \quad \partial_{n^{\varepsilon}} \mathcal{W}_{q j}^{\varepsilon}=\varepsilon^{2} \zeta_{j}^{\varepsilon} \mathcal{P}_{1} \text { on } \vartheta_{j}^{\varepsilon}=\partial \theta_{j}^{\varepsilon} \cap \Gamma^{\varepsilon}
$$

and put

$$
\mathcal{W}_{q}^{\varepsilon}=\sum_{j=1}^{N} \widehat{\zeta}_{j}^{\varepsilon} \mathcal{W}_{q j}^{\varepsilon},
$$

where $\widehat{\zeta}_{j}^{\varepsilon} \in C_{c}^{\infty}(\varepsilon j-\varepsilon / 2, \varepsilon j+3 \varepsilon / 2)$ and $\widehat{\zeta}_{j}^{\varepsilon} \zeta_{j}^{\varepsilon}=\zeta_{j}^{\varepsilon}$. This finishes the construction of the required function; it remains to estimate its Sobolev norms. The consideration of the data (3.31) is done conveniently in terms of coordinates that are $\varepsilon^{-1}$ times dilated relative to the mass center of $\theta_{j}^{\varepsilon}$. This operation of dilation will be denoted by the symbol b. The norm of the function $\varepsilon^{3}\left(\zeta_{j}^{\varepsilon} \mathcal{P}_{1}\right)_{b}$ in the Sobolev-Slobodetskiu space $H^{1 / 2}$ is $O\left(\varepsilon^{3}\right)$ (the additional factor $\varepsilon$ is due to the coordinate dilation). Therefore, the $H^{2}$-norm of the function $\left(\mathcal{W}_{q j}^{\varepsilon}\right)_{b}$ does not exceed $c \varepsilon^{3}$, and $c$ can be taken to be one and the same for all cells because the cells differ little from one another (see above). Upon returning to the initial coordinates, the square of the $H^{2}$-norm acquires the factor $\varepsilon^{2}\left(\varepsilon^{-2}\right)^{2}=\varepsilon^{-2}$; hence, 
after summing as in (3.32) and recalling that $N=1 / \varepsilon$, we obtain the majorant $c \varepsilon^{6-2-1}$ for the sum in question, together with the desired estimate (3.18) with the exponent $\gamma_{2}=3 / 2$ for the norm itself of the function (3.32).

We turn to handling the quantity (3.7) that occurs in Lemma 3.1 concerning near eigenvalues and eigenvectors; as an approximate eigenvalue $\mathcal{M}_{q}^{\varepsilon}$ in that lemma, we take the expression (3.9), and the role of an approximate eigenfunction will be played by

$$
\mathcal{U}_{q}^{\varepsilon}=\left\|\mathbf{U}_{q}^{\varepsilon} ; H^{\varepsilon}\right\|^{-1} \mathbf{U}_{q}^{\varepsilon}
$$

where $\mathbf{U}_{q}^{\varepsilon}$ is the asymptotic construction (3.17). By the definitions (3.1) and (3.2), we have

$$
\begin{aligned}
\delta_{q}^{\varepsilon} & =\left\|K^{\varepsilon} \mathcal{U}_{q}^{\varepsilon}-\mathcal{M}_{q}^{\varepsilon} \mathcal{U}_{q}^{\varepsilon} ; H^{\varepsilon}\right\| \\
& =\left\|\mathbf{U}_{q}^{\varepsilon} ; H^{\varepsilon}\right\|^{-1} \mathcal{M}_{q}^{\varepsilon} \sup \left|\left(\lambda_{p}+\varepsilon^{\gamma_{0}} \lambda_{q}^{\prime}\right)\left(\mathbf{U}_{q}^{\varepsilon}, V^{\varepsilon}\right)_{\Omega^{\varepsilon}}-\left(\Delta_{x} \mathbf{U}_{q}^{\varepsilon}, \Delta_{x} V^{\varepsilon}\right)_{\Omega^{\varepsilon}}\right| \\
& \leq c \sup \left|\left(\Delta_{x}^{2} \mathbf{U}_{q}^{\varepsilon}-\left(\lambda_{p}+\varepsilon^{\gamma_{0}} \lambda_{q}^{\prime}\right) \mathbf{U}_{q}^{\varepsilon}, V^{\varepsilon}\right)_{\Omega^{\varepsilon}}\right| .
\end{aligned}
$$

Here the supremum is taken over all $V^{\varepsilon} \in H^{\varepsilon}$ such that $\left\|V^{\varepsilon} ; H^{\varepsilon}\right\|=1$, and in the last line in (3.33) we used the fact that, by (3.9) and (3.28), the quantity $\left\|\mathbf{U}_{q}^{\varepsilon} ; H^{\varepsilon}\right\|^{-1} \mathcal{M}_{q}^{\varepsilon}$ is uniformly bounded for $\varepsilon \in\left(0, \varepsilon_{p}\right]$ with $\varepsilon_{p}>0$. Observe that, by the inequalities (1.6) and (4.12), we have

$$
\left\|\rho_{\varepsilon}^{-2} V^{\varepsilon} ; L_{2}\left(\Omega^{\varepsilon}\right)\right\|+\left\|\rho_{\varepsilon}^{-1} \nabla_{x} V^{\varepsilon} ; L_{2}\left(\Omega^{\varepsilon}\right)\right\|+\left\|\nabla_{x}^{2} V^{\varepsilon} ; L_{2}\left(\Omega^{\varepsilon}\right)\right\| \leq c .
$$

Estimate (3.18) shows that the correction term $\mathcal{W}_{q}^{\varepsilon}$ can be excluded from further consideration, because

$$
\left|\left(\lambda_{p}+\varepsilon^{\gamma_{0}} \lambda_{q}^{\prime}\right)\left(\mathcal{W}_{q}^{\varepsilon}, V^{\varepsilon}\right)_{\Omega^{\varepsilon}}-\left(\Delta_{x} \mathcal{W}_{q}^{\varepsilon}, \Delta_{x} V^{\varepsilon}\right)_{\Omega^{\varepsilon}}\right| \leq c \varepsilon^{\gamma_{2}} .
$$

We perform transformations similar to (3.20):

$$
\begin{aligned}
\Delta_{x}^{2} & \left(\mathcal{X}_{\varepsilon} S_{o}^{\varepsilon}+\chi S_{i}^{\varepsilon}-\mathcal{X}_{\varepsilon} \chi S_{m}^{\varepsilon}\right)-\left(\lambda_{p}+\varepsilon^{\gamma_{0}} \lambda_{q}^{\prime}\right)\left(\mathcal{X}_{\varepsilon} S_{o}^{\varepsilon}+\chi S_{i}^{\varepsilon}-\mathcal{X}_{\varepsilon} \chi S_{m}^{\varepsilon}\right) \\
= & \mathcal{X}_{\varepsilon}\left(\Delta_{x}^{2} S_{o}^{\varepsilon}-\left(\lambda_{p}+\varepsilon^{\gamma_{0}} \lambda_{q}^{\prime}\right) S_{o}^{\varepsilon}\right)+\left[\Delta_{x}^{2}, \mathcal{X}_{\varepsilon}\right]\left(S_{0}^{\varepsilon}-S_{m}^{\varepsilon}\right) \\
& +\chi\left(\Delta_{x}^{2} S_{i}^{\varepsilon}-\mathcal{X}_{\varepsilon} \Delta_{x}^{2} S_{m}^{\varepsilon}\right)-\left(\lambda_{p}+\varepsilon^{\gamma_{0}} \lambda_{q}^{\prime}\right) \chi\left(S_{i}^{\varepsilon}-\mathcal{X}_{\varepsilon} S_{m}^{\varepsilon}\right)+\left[\Delta_{x}^{2}, \chi\right]\left(S_{i}^{\varepsilon}-S_{m}^{\varepsilon}\right) \\
= & : \mathcal{X}_{\varepsilon} \Sigma_{o}^{\varepsilon}+\Sigma_{o m}^{\varepsilon}+\chi \Sigma_{i m}^{\varepsilon}-\chi \Sigma_{\lambda}^{\varepsilon}+\Sigma_{\chi}^{\varepsilon}
\end{aligned}
$$

Here, we encounter commutators of the biharmonic operator with the cutoff functions (3.17), but formulas similar to (3.19) remain valid. The commutator $\left[\Delta_{x}^{2}, \chi\right]$ is a thirdorder differential operator with smooth coefficients, the supports of which are $O(d)$ distant from the boundary $\Gamma^{\varepsilon}$. The coefficients of the derivatives of order $p=0,1,2,3$ in the commutator $\left[\Delta_{x}^{2} ; \mathcal{X}_{\varepsilon}\right]$ are $O\left(\varepsilon^{-4+p}\right)$, and their supports are located in the $d \varepsilon-$ neighborhood of $\Gamma$. We shall use these properties in the further calculations.

Recalling equations (1.9) and (2.12), we see that

$$
\Sigma_{o}^{\varepsilon}=\left(\Delta_{x}^{2} u_{q}^{0}-\lambda_{p} u_{q}^{0}\right)+\varepsilon^{\gamma_{0}}\left(\Delta_{x}^{2} u_{q}^{\prime}-\lambda_{p} u_{q}^{\prime}-\lambda_{q}^{\prime} u_{q}^{0}\right)-\varepsilon^{2 \gamma_{0}} \lambda_{q}^{\prime} u_{q}^{0}=-\varepsilon^{2 \gamma_{0}} \lambda_{q}^{\prime} u_{q}^{0},
$$

i.e.,

$$
\left|\left(\mathcal{X}^{\varepsilon} \Sigma_{o}^{\varepsilon}, V^{\varepsilon}\right)_{\Omega^{\varepsilon}}\right| \leq c \varepsilon^{2 \gamma_{0}} .
$$

We repeat the transformations (3.21) with obvious modifications and use the weight inequality (3.34) and what was said above about the commutator $\left[\Delta_{x}^{2}, \mathcal{X}_{\varepsilon}\right]$ to show that (3.38)

$$
\left|\left(\Sigma_{o m}^{\varepsilon}, V^{\varepsilon}\right)_{\Omega^{\varepsilon}}\right| \leq c\left\|\rho_{\varepsilon}^{-2} V_{\varepsilon} ; L_{2}\left(\Omega^{\varepsilon}\right)\right\|\left(\int_{d \varepsilon}^{0}(|n|+\varepsilon)^{4}\left(|n|^{3-p}+\varepsilon|n|^{(2-p)_{+}}\right)^{2} d n\right)^{1 / 2} \leq c \varepsilon^{3 / 2}
$$

here $(t)_{+}=(|t|+t) / 2$ is the positive part of $t \in \mathbb{R}$. 
Now we consider the terms that arose in (3.36) because of the internal expansion (2.34). We begin with the simpler, the last, term. Since the difference (3.22) is bounded with respect to $\xi_{1}$ and involves the factor $\varepsilon^{2}$, we have

$$
\left|\left(\chi \Sigma_{\lambda}^{\varepsilon}, V^{\varepsilon}\right)_{\Omega^{\varepsilon}}\right| \leq c \varepsilon^{2}\left\|V^{\varepsilon} ; L_{2}\left(\Omega^{\varepsilon}\right)\right\| \leq c \varepsilon^{2} .
$$

Formula (1.14) for the Laplacian allows us to rewrite the biharmonic operator in the curvilinear coordinates: $\Delta_{x}^{2}=\mathcal{L}\left(n, s, \partial_{n}, \partial_{s}\right)$. Also, note that

$$
\partial_{s} W_{0}\left(\varepsilon^{-1} n, \varepsilon^{-1} s, s\right)=\left.\left(\frac{\partial W_{0}}{\partial s}(\xi, s)+\frac{1}{\varepsilon} \frac{\partial W_{0}}{\partial \xi_{2}}(\xi, s)\right)\right|_{\xi=\varepsilon^{-1}(n, s)} .
$$

Thus, we can write

$$
\begin{aligned}
& \mathcal{L}\left(n, s, \partial_{n}, \partial_{s}\right) W_{0}\left(\varepsilon^{-1} n, \varepsilon^{-1} s, s\right) \\
& \quad=\left.\left(\varepsilon^{-4} \Delta_{\xi} W_{0}(\xi, s)+\widetilde{\mathcal{L}}_{\varepsilon}\left(n, s, \nabla_{(n, s)}, \nabla_{\xi}\right) W_{0}(\xi, s)\right)\right|_{\xi=\varepsilon^{-1}(n, s)}
\end{aligned}
$$

where $\widetilde{\mathcal{L}}_{\varepsilon}$ is a fourth-order operator for which the coefficients of the derivatives $\nabla_{\xi}^{4}$ are $O\left(\varepsilon^{-4}|n|\right)$, and those of the derivatives $\nabla_{(n, s)}^{j} \nabla_{\xi}^{k}$ are $O\left(\varepsilon^{-k}\right)$; here $j=0, \ldots, 4, k=$ $0, \ldots, 3$, and $j+k \leq 4$.

We agree to use the slow and fast variables simultaneously, which is customary in homogenization theory (cf. relations (3.40) ). Also, we assume that in the expression $S_{m}^{\varepsilon}$ the coordinate $n$ is replaced with the dilated coordinate $\xi_{1}=\varepsilon^{-1} n$, i.e., $S_{m}^{\varepsilon}(x)=$ $\varepsilon^{2} \partial_{n}^{2} u_{q}^{0}(0, s)\left(\frac{1}{2} \xi_{1}^{2}+P(s) \xi_{1}\right)$. Then for both terms we obtain $\Delta_{\xi}^{2} S_{i}^{\varepsilon}=0$ and $\Delta_{\xi}^{2} S_{m}^{\varepsilon}=0$. In the difference $S^{\varepsilon}(x)-S_{m}^{\varepsilon}(x)$, the quadratic terms $O\left(\xi_{1}^{2}\right)$, as well as the linear terms $O\left(\left|\xi_{1}\right|\right)$, mutually cancel, and the constant term $P_{01}(s)$ (cf. (3.22) ) gives rise to a smooth function $x \mapsto \varepsilon^{2} \chi(x) \Delta_{x}^{2} \partial_{n} u_{q}^{0}(0, s) P_{01}(s)$, for which the required estimate is obvious due to the factor $\varepsilon^{2}$. As a result, since the remainder term (3.23) decays exponentially (see Proposition 2.1), we conclude that

$$
\begin{aligned}
& \left|\left(\chi \Sigma_{i m}^{\varepsilon}, V^{\varepsilon}\right)_{\Omega^{\varepsilon}}\right| \leq c \varepsilon^{2}\left\|\rho_{\varepsilon}^{-2} V^{\varepsilon} ; L_{2}\left(\Omega^{\varepsilon}\right)\right\| \\
& \quad \times\left(1+\sum_{k=0}^{4} \varepsilon^{-2 k} \int_{-d}^{c \varepsilon}(|n|+\varepsilon)^{4} n^{2 \delta_{k, 4}} \exp \left(2 \delta \frac{n}{\varepsilon}\right) d n\right)^{1 / 2} \leq c \varepsilon^{3 / 2} .
\end{aligned}
$$

It remains to consider the summand $\Sigma_{\chi}^{\varepsilon}$ with the commutator $\left[\Delta_{x}^{2}, \chi\right]$. Much as in the proof of the second relation in (3.24), we arrive at the following result:

$$
\left|\left(\Sigma_{x}^{\varepsilon}, V^{\varepsilon}\right)_{\Omega^{\varepsilon}}\right| \leq c \varepsilon^{2}
$$

Now, combining formula (3.33) with estimates (3.35), (3.37)-3.39) and (3.41), (3.42) for the terms in (3.36), we see that $\delta_{q}^{\varepsilon} \leq c \varepsilon^{3 / 2}$. Thus, the first part of Lemma 3.1 ensures the existence of an eigenvalue in the sequence (3.5) for which

$$
\left|\mu_{j(q)}^{\varepsilon}-\left(\lambda_{p}+\varepsilon \lambda_{q}^{\prime}\right)^{-1}\right| \leq c_{p} \varepsilon^{3 / 2} .
$$

Suppose the eigenvalue $\lambda_{p}$ is simple, i.e., $\varkappa_{p}=1$, or all the eigenvalues of the matrix $\mathbf{T}^{p}$ are simple, i.e., all inequalities in (2.16) are strict, and hence, the segments on which, in accordance with (3.43), the numbers $\mu_{j(p)}^{\varepsilon}, \ldots, \mu_{j\left(p+\varkappa_{p}-1\right)}^{\varepsilon}$ lie, are disjoint. Then we have $\varkappa_{p}$ eigenvalues of the operator $K^{\varepsilon}$ that satisfy (3.12), so that the arguments of Subsection 2 in $\S 3$ lead to the required theorem on asymptotic formulas. However, in the case of a multiple eigenvalue,

$$
\lambda_{l-1}^{\prime}<\lambda_{l}^{\prime}=\cdots=\lambda_{l+\varrho_{l}-1}^{\prime}<\lambda_{l+\varrho_{l}}^{\prime}
$$

(cf. formula (1.17)), we also need to verify that there exist $\varrho_{l}>1$ distinct eigenvalues of $K^{\varepsilon}$ that satisfy (3.43) for $q=l, \ldots, l+\varrho_{l}-1$. 
Let $\mu_{L^{\varepsilon}}^{\varepsilon}, \ldots, \mu_{L^{\varepsilon}+N^{\varepsilon}-1}^{\varepsilon}$ be all the eigenvalues of the operator $K^{\varepsilon}$ on the segment

$$
I_{l}^{\varepsilon}(\beta)=\left[\left(\lambda_{p}+\varepsilon \lambda_{l}^{\prime}\right)^{-1}-\beta c_{p} \varepsilon^{3 / 2},\left(\lambda_{p}+\varepsilon \lambda_{l}^{\prime}\right)^{-1}+\beta c_{p} \varepsilon^{3 / 2}\right],
$$

where $\beta$ is a (generally speaking, large) number to be chosen later, and $\varepsilon$ is so small that the segment (3.44) does not intersect any other such segment $I_{q}^{\varepsilon}(\alpha)$ with $q \neq l, \ldots, l+$ $\varrho_{l}-1$. In Lemma 3.1, put $\triangle_{l}^{\varepsilon}=\alpha c_{p} \varepsilon^{3 / 2}$. Then there exist coefficient columns $A_{(m)}^{\varepsilon}=$ $\left(A_{m, l}^{\varepsilon}, \ldots, A_{m, l+\tau_{l}-1}^{\varepsilon}\right)$ of unit height (see the second formula in (3.8) $)$ such that

$$
\left\|\mathcal{U}_{m}^{\varepsilon}-\sum_{j=L^{\varepsilon}}^{L^{\varepsilon}+T^{\varepsilon}-1} A_{m, j}^{\varepsilon} U_{j}^{\varepsilon} ; H^{\varepsilon}\right\| \leq 2 \frac{\delta_{m}^{\varepsilon}}{\triangle_{m}^{\varepsilon}} \leq \frac{2}{\beta} .
$$

For $m \neq n$, with the help of (3.27) and (1.11) we get

$$
\left|\left\langle\mathcal{U}_{m}^{\varepsilon}, \mathcal{U}_{n}^{\varepsilon}\right\rangle\right|=\left\|\mathbf{U}_{m}^{\varepsilon} ; H^{\varepsilon}\right\|^{-1}\left\|\mathbf{U}_{n}^{\varepsilon} ; H^{\varepsilon}\right\|^{-1}\left|\left\langle\mathbf{U}_{m}^{\varepsilon}, \mathbf{U}_{n}^{\varepsilon}\right\rangle\right| \leq c \varepsilon^{\gamma_{0} / 2}
$$

On the other hand, by the orthogonality and normalization conditions (3.6), we have

$$
\begin{aligned}
& \left|\sum_{j=L^{\varepsilon}}^{L^{\varepsilon}+T^{\varepsilon}-1} A_{m, j}^{\varepsilon} A_{n, j}^{\varepsilon}\right|=\left|\left\langle\sum_{j=L^{\varepsilon}}^{L^{\varepsilon}+T^{\varepsilon}-1} A_{m, j}^{\varepsilon} \mathbf{U}_{j}^{\varepsilon}, \sum_{j=L^{\varepsilon}}^{L^{\varepsilon}+T^{\varepsilon}-1} A_{n, j}^{\varepsilon} \mathbf{U}_{j}^{\varepsilon}\right\rangle\right| \\
& \quad=\left|\left\langle\Sigma_{m}^{\varepsilon}, \Sigma_{n}^{\varepsilon}\right\rangle\right|=\left|\left\langle\Sigma_{m}^{\varepsilon}-\mathcal{U}_{m}^{\varepsilon}, \Sigma_{m}^{\varepsilon}\right\rangle+\left\langle\mathcal{U}_{m}^{\varepsilon}, \Sigma_{n}^{\varepsilon}-\mathcal{U}_{n}^{\varepsilon}\right\rangle+\left\langle\mathcal{U}_{m}^{\varepsilon}, \mathcal{U}_{n}^{\varepsilon}\right\rangle\right| \\
& \quad \leq 2 \beta^{-1}+2 \beta^{-1}+c \varepsilon^{\gamma_{0} / 2} .
\end{aligned}
$$

Here $\Sigma_{m}^{\varepsilon}$ is the sum under the norm sign in (3.45). Recalling the second formula in (3.8), we see that, for large $\beta$ and sufficiently small $\varepsilon$, the columns $A_{l+\varrho_{l}-1}^{\varepsilon} \in \mathbb{R}^{T^{\varepsilon}}$ are "almost orthonormalized", which is possible only if $\varrho_{l} \leq T^{\varepsilon}$.

This establishes the required claim; hence, Theorem 3.1 is proved in the case where $\gamma=1$.

Remark 3.1. The resulting estimate for $\tilde{\lambda}_{q}^{\varepsilon}$ turns out to be asymptotically sharp: the rule of "the first discarded term" works. Namely, in the Ansatz (2.24), and then also in the construction (3.16), the term $\chi(x) \varepsilon^{3} w^{\prime \prime}\left(\varepsilon^{-1}, \varepsilon^{-1} s, s\right)$ of the boundary layer type was not taken into account, and the norm of this term in the space $H^{2}\left(\Omega^{\varepsilon}\right)$ is precisely $O\left(\varepsilon^{3 / 2}\right)$.

5. Discrepancy estimate for $\gamma<1$. In essence, the only difficulty is to estimate the correction term $\mathcal{W}^{\varepsilon}$ in (3.16), at least because now the boundary condition (3.29) becomes nonhomogeneous, and the right-hand sides of (3.29) and (3.30) are calculated in accordance with the expansions (2.42) near the vertex of the check (2.38).

Remark 3.2. Due to the assumption (2.35), the leading terms of equation (2.37) of the boundary take the form $\xi_{1}=\varepsilon^{\gamma-1} A\left(s_{0}\right)\left(\xi_{2}-\eta_{0}\left(s_{0}\right)\right)^{2}$. Therefore, passage to the "superfast" variables

$$
\widehat{\xi}=\left(\widehat{\xi}_{1}, \widehat{\xi}_{2}\right)=\left(\varepsilon^{\gamma-1} \xi_{1}, \varepsilon^{\gamma-1}\left(\xi_{2}-\eta_{0}\left(s_{0}\right)\right)\right.
$$

transforms the domain $\Omega^{\varepsilon}$ inside a small neighborhood of the point $\left(s_{0}, \varepsilon^{\gamma} H\left(s_{0}, \eta_{0}\left(s_{0}\right)\right)\right)$ to the exterior of the parabola $\hat{\xi}_{1}=A\left(s_{0}\right)\left(\hat{\xi}_{2}\right)^{2}$. As a result, the phenomenon of a "deep" boundary layer arises, described in 54 for a second-order elliptic equation. We shall not need such a boundary layer for justifying the leading terms of the asymptotic expansion. In the case of a nonstrict minimum, if we replace (2.35) by the condition

$$
\begin{array}{r}
\left.H\left(\eta, s_{0}\right)=A\left(s_{0}\right)\left(\eta-\eta_{0}\left(s_{0}\right)\right)^{2 m}+\left.O\left(\mid \eta-\eta_{0}\left(s_{0}\right)\right)\right|^{2 m+1}\right), \\
A(s)>0, \quad m \in \mathbb{N}, \quad m>1,
\end{array}
$$

then $\varepsilon^{\gamma-1}$ appears in place of the dilatation coefficient $\varepsilon^{(\gamma-1) /(2 m-1)}$. 
Let $s \in(\varepsilon(j-1), \varepsilon j)$ for some $j=1, \ldots, N$. Since the profile function is smooth in both arguments, the points $\left(\varepsilon^{\gamma} H_{0}\left(\eta_{0}(s), s\right), s\right)$ ran over an arc of length $O\left(\varepsilon^{1+\gamma}\right)$ (cf. (1.16)). We introduce a cutoff function $\chi_{\varepsilon^{1+\gamma}}^{0}$ with support in the $2 c \varepsilon^{1+\gamma}$-neighborhood of that arc and equal to 1 in the $c \varepsilon^{1+\gamma}$-neighborhood. It is not hard to ensure the relation $\left|\nabla_{x}^{\ell} \chi_{\varepsilon^{1+\gamma}}^{0}(x)\right| \geq c_{\ell} \varepsilon^{-\ell(1+\gamma)}$; hence, by (2.42), we have

$$
\begin{aligned}
& \left\|\chi_{\varepsilon^{1+\gamma}}^{0} \varepsilon^{2} \partial_{n} u_{q}^{0} W_{0} ; H^{2}\left(\Omega^{\varepsilon}\right)\right\|^{2} \\
& \quad \leq c \varepsilon^{4} \int_{0}^{\varepsilon^{1+\gamma}}\left(\varepsilon^{-4(1+\gamma)}\left(\varepsilon^{\gamma}+\varepsilon^{-1} r\right)^{3}+\varepsilon^{-4}\left(\varepsilon^{\gamma}+\varepsilon^{-1} r\right)^{-1}\right) r d r \leq c \varepsilon^{2+\gamma} .
\end{aligned}
$$

In other words, near the points of minimum of the profile function, for the role of the correction $\mathcal{W}_{q}^{\varepsilon}$ we can take the sum $\mathcal{W}_{q \text {,min }}^{\varepsilon}$ of the functions occurring under the norm sign on the left in (3.47). We have $N=1 / \varepsilon$ such functions, whence

$$
\left\|\mathcal{W}_{q, \text { min }}^{\varepsilon} ; H^{2}\left(\Omega^{\varepsilon}\right)\right\|^{2} \leq c\left(N \varepsilon^{2+\gamma}\right)^{1 / 2}=c \varepsilon^{(1+\gamma) / 2} .
$$

Relations (2.42) and (2.40) yield the following estimates in the strip $\mathbb{R} \times(0,1)$ :

$$
\begin{aligned}
\left|W_{0}(\xi)\right| & \leq c \rho^{3 / 2}\left|\rho^{-1} \xi_{2}\right|^{2} \exp \left(-\delta_{1} \xi_{1}\right)=c \rho^{-1 / 2}\left|\xi_{2}\right|^{2} \exp \left(-\delta_{1} \xi_{1}\right), \\
\left|\nabla_{\xi} W_{0}(\xi)\right| & \leq c \rho^{-1 / 2}\left|\xi_{2}\right| \exp \left(-\delta_{1} \xi_{1}\right), \\
\left|\nabla_{\xi}^{2} W_{0}(\xi)\right| & \leq c \rho^{-1 / 2} \exp \left(-\delta_{1} \xi_{1}\right) .
\end{aligned}
$$

Note that the factor $\left|\rho^{-1} \xi_{2}\right|^{2} \sim(|\phi|-\pi)^{2}$ has arisen because of the double zero of the angular part $3 \sin (\phi / 2)+\sin (3 \phi / 2)$ at the points $\phi= \pm \pi$, i.e., on the shores of the cut $\Upsilon=\left\{\xi: \xi_{2}=0, \xi \geq 0\right\}$. The presence of an exponential factor on the right-hand sides of (3.48) allows us to use the sum $\mathcal{W}_{q, \max }^{\varepsilon}$ of the products $\varepsilon^{2} \partial_{n} u_{q}^{0} W_{0}$ multiplied by $1-\chi_{\varepsilon^{1+\gamma}}^{0}$ for the role of $\mathcal{W}_{q}^{\varepsilon}$ on the fragments $\left\{x \in \Gamma^{\varepsilon}: n>H_{0}(s)+c \varepsilon^{1+\gamma}\right\}$ ("offcuts of petals"). Then

$$
\left\|\mathcal{W}_{q, \max }^{\varepsilon} ; H^{2}\left(\Omega^{\varepsilon}\right)\right\|^{2} \leq c \exp \left(-\delta_{2} \varepsilon^{-\gamma}\right), \quad \delta_{2} \in\left(0, \delta_{1}\right)
$$

As a result, we arrive at the required estimate (3.18). In essence, the remaining part of the proof repeats the arguments of the preceding subsection; the necessary but insignificant modifications were already discussed in Subsection 3 of $\S 4$. Thus, we assume that Theorem 3.1, 1), is proved for $\gamma<1, H_{0} \not \equiv 0$.

6. The remaining two cases. In the case of a slanting $(\gamma>1)$ perturbation of the boundary, the term of the boundary layer type can be excluded from the construction of the asymptotic approximation (3.16); this formula becomes

$$
\mathbf{U}_{q}^{\varepsilon}(x)=u_{q}^{0}(x)+\varepsilon^{\gamma} u_{q}^{\prime}(x)+\mathcal{W}_{q}^{\varepsilon}(x) .
$$

The functions $u_{q}^{0}$ and $u_{q}^{\prime}$ are defined by formulas (2.3) and (2.12), (2.11), and the boundary condition for $u_{q}^{\prime}$ involves the mean value $\bar{H}(s)$ of the profile function. The small correction term $\mathcal{W}_{q}^{\varepsilon}$ must compensate for the smooth type discrepancies in the boundary conditions (2.5) and (2.6). Recalling that $u_{q}^{0}$ and $u_{q}^{\prime}$ are extended smoothly outside of $\Omega$, we arrive at the relation

$$
\begin{aligned}
& u_{q}^{0}\left(\varepsilon^{\gamma} H\left(\varepsilon^{-1} s, s\right), s\right)+\varepsilon^{\gamma} u_{q}^{\prime}\left(\left(\varepsilon^{\gamma} H\left(\varepsilon^{-1} s, s\right), s\right)\right. \\
& \quad=\varepsilon^{2 \gamma}\left(\frac{1}{2} H\left(\varepsilon^{-1} s, s\right)^{2} \partial_{n}^{2} u_{q}^{0}(0, s)+H\left(\varepsilon^{-1} s, s\right) u_{q}^{\prime}(0, s)\right)+O\left(\varepsilon^{3 \gamma}\right) .
\end{aligned}
$$

If we act as in Subsection 4 of $\S 3$, and choose any function $\mathcal{W}_{q}^{\varepsilon} \in H^{2}\left(\Omega^{\varepsilon}\right)$ that takes the value opposite to (3.49) on the boundary $\Gamma^{\varepsilon}$, then it will satisfy estimate (3.18) with the exponent $\gamma_{2}=2 \gamma-3 / 2>0$. This estimate suffices for proving the auxiliary formulas such as (3.27) and (3.28) with infinitesimally small majorants, but it is unsuitable for proving Theorem 3.1, because the exponent $\gamma_{1}=2 \gamma-1 / 2$ in the second formula in 
(3.11) turns out to be greater than the $\gamma_{2}$ obtained. Looking through what was said in Subsection 4 of $\S 3$ once again, we find that relation (3.18) was employed to deduce inequality (3.35), and that this inequality (and only it) leads to an inadmissible enlarging of the majorant for the quantity (3.33) in Lemma 3.1.

This situation can be remedied by imitation of the boundary layer phenomenon as follows. We compensate for the averages over the fast variable $\xi_{2}=\varepsilon^{-1} s$ of the righthand sides in (3.49) and in a similar identity for $\partial_{n^{\varepsilon}}\left(u_{q}^{0}+\varepsilon^{\gamma} u_{q}^{\prime}\right)$ with the help of a function depending on the slow variables, and the remainder will be compensated for with the help of the solution of problem (2.21) - 2.23) in the half-strip $\Pi_{-}$with the rectangular edge $\varpi=\left\{\xi: \xi_{1}=0, \xi_{2} \in(0,1)\right\}$. Since in this special case we have $W_{0}(\xi)=\xi_{1}^{2} / 2$ and $W_{0}(\xi)=\xi_{1}^{3} / 6$, Proposition 2.1 2) shows that the resulting Ersatz of the boundary layer decays exponentially, because the right-hand sides $g_{0}$ and $g_{1}$ have zero mean. Now, by repeating the calculations that led in Subsection 4 of $\S 3$ to inequality (3.38), we see that $\delta_{q}^{\varepsilon} \leq c \varepsilon^{2 \gamma-1 / 2}$ (cf. formula (3.11)).

Similar modifications are needed in the case where $\gamma<1, H_{0} \equiv 0$, because relation (3.11) does not provide the required majorant in (3.35). What is needed is the "deep" boundary layer employing the superfast variables (3.46) and described in Remark 3.2 . With such a boundary layer in the construction of the correction term $\mathcal{W}_{q}^{\varepsilon}$, we get the desired relation $\delta_{q}^{\varepsilon} \leq c \varepsilon^{1+\gamma / 2}$.

In the last two cases, the resulting estimates turn out to be asymptotically sharp in the sense of Remark 3.1. However, the estimate found in the case of $\gamma<1, H_{0} \not \equiv 0$ (see Subsection 5 of $\S 3$ ) fails to possess this property, again because the deep boundary layer was not taken into account in the correction term $\mathcal{W}_{q}^{\varepsilon}$.

7. Some words about the asymptotic approximations of eigenfunctions. The standard approach (see, e.g., [52, 9, 4]) allows us to use the second part of Lemma 3.1 on "near eigenvalues and eigenvectors" for justifying the asymptotic formulas (2.2) and (2.24) for $u_{p}^{\varepsilon}, \ldots, u_{p+\varkappa_{p}-1}^{\varepsilon}$. Naturally, some loss of accuracy occurs: recall that a smooth type correction term is not unique, being determined only up to a linear combination of the "limiting eigenfunctions" $u_{p}, \ldots, u_{p+\varkappa_{p}-1}$, which are unknown at that stage of the asymptotic procedure. In Theorem 3.1, we presented a simpler result, in which the boundary layer is neglected, so that the majorant is somewhat enlarged additionally.

\section{§4. NonRegular Perturbation of the Boundary}

1. On the notation. In accordance with [7, the content of this section is closely related to the abstract setting (3.3) of problem (1.5), dealing with the eigenvalues (3.5) of the operator $K^{\varepsilon}$. Therefore, all asymptotic expansions are formulated for the eigenvalues $\mu_{p}^{\varepsilon}=1 / \lambda_{p}^{\varepsilon}$. Of course, such formulas can easily be reshaped into asymptotic representations of the eigenvalues $\lambda_{p}^{\varepsilon}$ themselves; see (3.13) (cf. Subsection 1 of $\S 5$ ).

2. An abstract theorem. The proof of the results mentioned in Subsection 3 of $\S 1$ is based on an application of [7, Theorem 1], which makes it possible to compare the eigenvalues of two compact operators acting in different Hilbert spaces. The first pair space/operator that we need was already determined in Subsection 1 of $\S 3$ : the space

$H^{\varepsilon}=\stackrel{\circ}{H}^{2}\left(\Omega^{\varepsilon}\right)$ with the scalar product (3.1) and the operator $K^{\varepsilon}$ given by (3.2). Clear modifications of the definitions provide the space $H=H^{0}$, the scalar product $\langle\rangle=,\langle,\rangle_{0}$ and the operator $K=K^{0}$ with the same properties as $K^{\varepsilon}$, i.e., $K$ is compact, positive, and selfadjoint; relations (3.1) and (3.2) take the form

$$
\langle u, v\rangle=\left(\Delta_{x} u, \Delta_{x} v\right)_{\Omega}, \quad\langle K u, v\rangle=(u, v)_{\Omega}, \quad u, v \in H .
$$


The eigenvalues of $K$ will be denoted by $\mu_{1} \geq \mu_{2} \geq \cdots \geq \mu_{p} \geq \cdots \rightarrow+0$ (cf. (3.5)), and the variational spectral problem (1.11) is equivalent to the abstract equation $K u=\mu u$ in $H$, similar to (3.3), and the eigenvalues $\lambda_{p}$ and $\mu_{p}$ are related by (3.4).

In order to compare the eigenvalues $\mu_{q}$ and $\mu_{q}^{\varepsilon}$ of the operators $K$ and $K^{\varepsilon}$, we apply Proposition 1 and Theorem 1 of the paper [8. In the sequel, it is convenient to assume that the functions of classes $H$ and $H^{\varepsilon}$ are extended by zero to the entire plane. We shall need the operator $\mathfrak{S}: H^{0} \mapsto H^{\varepsilon}$ defined as follows: for $\varphi \in H^{0}$ by $\phi=\mathfrak{S} \varphi \in H^{\varepsilon}$ we mean a unique solution of the problem

$$
\langle\phi, v\rangle_{\varepsilon}=\langle\varphi, v\rangle_{\varepsilon}, \quad v \in H^{\varepsilon} .
$$

For the conjugate operator $\mathfrak{S}^{*}: H^{\varepsilon} \rightarrow H$ we have $\left\langle\varphi, \mathfrak{S}^{*} v\right\rangle=\langle\varphi, v\rangle, \varphi \in H$. By definition, $\left\|\mathfrak{S} ; H \rightarrow H^{\varepsilon}\right\|=\left\|\mathfrak{S}^{*} ; H^{\varepsilon} \rightarrow H\right\|=1$. Moreover, $\mathfrak{S} w=w$ and $\mathfrak{S}^{*} w=w$ whenever $w \in H \cap H^{\varepsilon}$. If $w^{\varepsilon} \in H^{\varepsilon}$ (or $w \in H$ ) and the vector $w^{\varepsilon}$ (or $w$ ) is orthogonal to $H \cap H^{\varepsilon}$, then the same orthogonality remains valid for $\mathfrak{S} w\left(\right.$ or $\left.\mathfrak{S}^{*} w^{\varepsilon}\right)$.

Compared to Subsection 1 of $\S 1$, now we characterize the mutual proximity of the domains $\Omega$ and $\Omega^{\varepsilon}$ by a more complicated condition, which will be verified afterwards starting with the previous requirements. Observe that if functions $w \in H$ and $w^{\varepsilon} \in H^{\varepsilon}$ are orthogonal to the subspace $H \cap H^{\varepsilon}$, then

$$
\left\|w ; L_{2}(\Omega)\right\|^{2} \leq \nu\left\|\nabla_{x}^{2} w ; L_{2}(\Omega)\right\|^{2}, \quad\left\|w^{\varepsilon} ; L_{2}\left(\Omega^{\varepsilon}\right)\right\|^{2} \leq \nu\left\|\nabla_{x}^{2} w ; L_{2}\left(\Omega^{\varepsilon}\right)\right\|^{2}
$$

with some constant $\nu$, which will be regarded as a small parameter in what follows. Namely, the new condition looks like this:

(i) $\Omega$ and $\Omega^{\varepsilon}$ are bounded domains with nonempty intersection, and the quantity $\nu$ in (4.1) is small.

We introduce the operator $\mathfrak{B}=K^{\varepsilon} \mathfrak{S}-\mathfrak{S} K: H \rightarrow H^{\varepsilon}$ and denote by $\varsigma$ the best constant in the inequality

$$
\left|\langle\mathfrak{B} \varphi, \mathfrak{S} \psi\rangle_{\varepsilon}\right| \leq \varsigma\|\varphi ; H\|\left\|\mathfrak{S} \psi ; H^{\varepsilon}\right\|, \quad \varphi, \psi \in \mathfrak{X}_{p} .
$$

Let $\mathfrak{Y}_{p}$ be the orthogonal complement of the subspace $\mathfrak{S X}_{p}$ in $H^{\varepsilon}$. Also, let $\mathfrak{P}_{p}$ denote the orthogonal projection onto $\mathfrak{S X}_{p}$ in $H^{\varepsilon}$, and let $\mathfrak{Q}_{p}=1-\mathfrak{P}_{p}$.

Lemma 4.1. Let $\lambda_{p}$ be an eigenvalue of problem (1.11) of multiplicity $\varkappa_{p}$ (see (1.17)). If the constant $\nu$ in inequalities (4.1) is small, then the interval (1.5) contains exactly $\varkappa_{p}$ eigenvalues of problem (1.18), and the following asymptotic formula is valid:

$$
\left(\lambda_{q}^{\varepsilon}\right)^{-1}=\lambda_{p}^{-1}+\mathfrak{t}_{q}+O(\nu(\varsigma+\nu)), \quad q=p, \ldots, p+\varkappa_{p}-1,
$$

where $\mathfrak{t}_{p}, \ldots, \mathfrak{t}_{p+\varkappa_{p}-1}$ are the eigenvalues of the algebraic problem

$$
\langle\mathfrak{B} \varphi, \mathfrak{S} \psi\rangle_{\varepsilon}+\left\langle\mathfrak{b}_{\varphi}, \mathfrak{B} \psi\right\rangle_{\varepsilon}=\mathfrak{t}\langle\mathfrak{S} \varphi, \mathfrak{S} \psi\rangle_{\varepsilon}, \quad \psi \in \mathfrak{X}_{p},
$$

for the unknown function $\varphi \in \mathfrak{X}_{p}$, and $\mathfrak{b}_{\varphi} \in \mathfrak{Y}_{p}$ is the solution of the equation

$$
\lambda_{p}^{-1} \mathfrak{b}_{\varphi}-\mathfrak{Q}_{p} K_{2} \mathfrak{b}_{\varphi}=\mathfrak{Q}_{p} \mathfrak{B} \varphi
$$

Proof. Essentially, the proof is the same as that of formula (53) in [7].

We show that any $\varphi \in \mathfrak{X}_{p}$ satisfies the inequality

$$
\left\|\mathfrak{B} \varphi ; \stackrel{\circ}{H}^{2}\left(\Omega^{\varepsilon}\right)\right\| \leq c \nu^{1 / 2}\left\|\varphi ; \stackrel{\circ}{H}^{2}\left(\Omega^{\varepsilon}\right)\right\| .
$$

Recalling the definitions of $K$ and $K^{\varepsilon}$, we see that

$$
\langle\mathfrak{B} \varphi, w\rangle_{\varepsilon}=(\mathfrak{S} \varphi, w)_{\Omega^{\varepsilon}}-\left(\varphi, \mathfrak{S}^{*} w\right)_{\Omega}
$$


for any test function $w \in \stackrel{\circ}{H}^{2}\left(\Omega^{\varepsilon}\right)$. Let $\varphi=\varphi_{0}+\varphi_{1}$ and $w=w_{0}+w_{1}$, where $\varphi_{0}, w_{0} \in$ $\stackrel{\circ}{H}^{2}\left(\Omega \cap \Omega^{\varepsilon}\right)$, and the components $\varphi_{1} \in \stackrel{\circ}{H}^{2}(\Omega)$ and $w_{1} \in \stackrel{\circ}{H}^{2}\left(\Omega^{\varepsilon}\right)$ are orthogonal to the subspace $\stackrel{\circ}{H}^{2}\left(\Omega \cap \Omega^{\varepsilon}\right)$. Then

$$
\langle\mathfrak{B} \varphi, w\rangle_{\varepsilon}=\left(\varphi_{0}, w_{1}\right)_{\Omega^{\varepsilon}}-\left(\varphi_{1}, w_{0}\right)_{\Omega}+\left(\mathfrak{S} \varphi_{1}, w\right)_{\Omega^{\varepsilon}}-\left(\varphi, \mathfrak{S}^{*} w_{1}\right)_{\Omega} .
$$

Applying inequalities (4.1) to $w_{1}, \mathfrak{S} \varphi_{1}$ and $\varphi_{1}, \mathfrak{S}^{*} w_{1}$, we obtain

$$
\left|\langle\mathfrak{B} \varphi, w\rangle_{\varepsilon}\right| \leq c \nu^{1 / 2}\|\varphi ; H\|\left\|w ; H^{\varepsilon}\right\| ;
$$

this implies the required estimate (4.6).

Now we compute the scalar product $\langle\mathfrak{B} \varphi, \mathfrak{S} \psi\rangle_{\varepsilon}$ occurring in (4.4). For $\varphi, \psi \in \mathfrak{X}_{p}$, we write

$$
\mathfrak{S} \varphi=\varphi+\Phi_{\varphi}^{\varepsilon}, \quad \mathfrak{S} \psi=\psi+\Phi_{\psi}^{\varepsilon},
$$

where $\Phi_{\varphi}^{\varepsilon}$ is the solution of problem (1.21). The function $\Phi_{\psi}^{\varepsilon}$ is introduced similarly. We have

$$
\langle\mathfrak{B} \varphi, \mathfrak{S} \psi\rangle_{\varepsilon}=\left(\varphi+\Phi_{\varphi}^{\varepsilon}, \psi+\Phi_{\psi}^{\varepsilon}\right)_{\Omega^{\varepsilon}}-\lambda_{p}^{-1}\left\langle\varphi+\Phi_{\varphi}^{\varepsilon}, \psi+\Phi_{\psi}^{\varepsilon}\right\rangle_{\varepsilon} .
$$

The definitions of $\Phi_{\varphi}^{\varepsilon}$ and $\Phi_{\psi}^{\varepsilon}$ imply that

$$
\left\langle\Phi_{\varphi}^{\varepsilon}, \psi+\Phi_{\psi}^{\varepsilon}\right\rangle_{\varepsilon}=\left\langle\varphi+\Phi_{\varphi}^{\varepsilon}, \Phi_{\psi}^{\varepsilon}\right\rangle_{\varepsilon}=0
$$

Thus,

$$
\langle\mathfrak{B} \varphi, \mathfrak{S} \psi\rangle_{\varepsilon}=\left(\varphi+\Phi_{\varphi}^{\varepsilon}, \psi+\Phi_{\psi}^{\varepsilon}\right)_{\Omega^{\varepsilon}}+\lambda_{p}^{-1}\left(\left\langle\Phi_{\varphi}^{\varepsilon}, \Phi_{\psi}^{\varepsilon}\right\rangle_{\varepsilon}-\langle\varphi, \psi\rangle_{\varepsilon}\right) .
$$

With the help of the relations

$$
\begin{aligned}
& \left(\Delta_{x} \varphi, \Delta_{x} \psi\right)_{\Omega \cap \Omega^{\varepsilon}}-\left(\Delta_{x} \varphi, \Delta_{x} \psi\right)_{\Omega \backslash \Omega^{\varepsilon}} \\
& \quad=\lambda_{p}(\varphi, \psi)_{\Omega \cap \Omega^{\varepsilon}}-\lambda_{p}(\varphi, \psi)_{\Omega \backslash \Omega^{\varepsilon}}+2 \int_{\Gamma_{0 \varepsilon}}\left(\Delta_{x} \varphi(x) \partial_{n} \psi(x)-\partial_{n} \Delta_{x} \varphi(x) \psi(x)\right) d s_{x}
\end{aligned}
$$

and

$$
\lambda_{p}\left(\varphi, \Phi_{\psi}\right)_{\Omega^{\varepsilon}}=\left\langle\varphi, \Phi_{\psi}\right\rangle_{\varepsilon}-\int_{\Gamma_{0 \varepsilon}}\left(\partial_{n} \Delta_{x} \varphi(x) \psi(x)-\Delta_{x} \varphi(x) \partial_{n} \psi(x)\right) d s_{x},
$$

where $\Gamma_{0 \varepsilon}=\partial\left(\Omega \cap \Omega^{\varepsilon}\right)$, we show that

$$
\begin{aligned}
\left\langle\varphi, \Phi_{\psi}^{\varepsilon}\right\rangle_{\varepsilon}=\lambda_{p}\left(\varphi, \Phi_{\psi}^{\varepsilon}\right)_{\Omega^{\varepsilon}}-\frac{1}{2}( & \left(\Delta_{x} \varphi, \Delta_{x} \psi\right)_{\Omega \cap \Omega^{\varepsilon}} \\
& \left.-\left(\Delta_{x} \varphi, \Delta_{x} \psi\right)_{\Omega \backslash \Omega^{\varepsilon}}-\lambda_{p}(\varphi, \psi)_{\Omega \cap \Omega^{\varepsilon}}+\lambda_{p}(\varphi, \psi)_{\Omega \backslash \Omega^{\varepsilon}}\right) .
\end{aligned}
$$

Together with a similar formula for $\left\langle\Phi_{\varphi}, \psi\right\rangle_{\varepsilon}$, this relation reshapes identity (4.10) as follows:

$$
\begin{aligned}
\langle\mathfrak{B} \varphi, \mathfrak{S} \psi\rangle_{\varepsilon}= & (\varphi, \psi)_{\Omega^{\varepsilon}}+\left(\Phi_{\varphi}^{\varepsilon}, \Phi_{\psi}^{\varepsilon}\right)_{\Omega^{\varepsilon}}+\lambda_{p}^{-1}\left(\left\langle\Phi_{\varphi}^{\varepsilon}, \Phi_{\psi}^{\varepsilon}\right\rangle_{\varepsilon}-\langle\varphi, \psi\rangle_{\varepsilon}\right) \\
& +\lambda_{p}^{-1}\left(\left\langle\varphi, \Phi_{\psi}^{\varepsilon}\right\rangle_{\varepsilon}+\left\langle\Phi_{\varphi}^{\varepsilon}, \psi\right\rangle_{\varepsilon}+\left(\Delta_{x} \varphi, \Delta_{x} \psi\right)_{\Omega \cap \Omega^{\varepsilon}}\right. \\
& \left.-\left(\Delta_{x} \varphi, \Delta_{x} \psi\right)_{\Omega \backslash \Omega^{\varepsilon}}-\lambda_{p}(\varphi, \psi)_{\Omega \cap \Omega^{\varepsilon}}+\lambda_{p}(\varphi, \psi)_{\Omega \backslash \Omega^{\varepsilon}}\right) .
\end{aligned}
$$

Applying (4.9), finally we deduce that

$$
\begin{aligned}
\left(\Delta_{x} \mathfrak{B} \varphi, \Delta_{x} \mathfrak{S} \psi\right)_{\Omega^{\varepsilon}}= & (\varphi, \psi)_{\Omega \backslash \Omega^{\varepsilon}}+\left(\Phi_{\varphi}^{\varepsilon}, \Phi_{\psi}^{\varepsilon}\right)_{\Omega^{\varepsilon}} \\
& -\lambda_{p}^{-1}\left(\left(\Delta_{x} \Phi_{\varphi}^{\varepsilon}, \Delta_{x} \Phi_{\psi}^{\varepsilon}\right)_{\Omega^{\varepsilon}}+\left(\Delta_{x} \varphi, \Delta_{x} \psi\right)_{\Omega \backslash \Omega^{\varepsilon}}\right) .
\end{aligned}
$$


4. Consequences of the requirements of Subsection 1 in $\S 1$, and estimation of the factor $\nu$. Assume the restrictions (1.2). We introduce the domains $\Omega_{+}^{\varepsilon}=\{x$ : $\operatorname{dist}(x, \Omega)<\varepsilon\}$ and $\Omega_{-}^{\varepsilon}=\{x \in \Omega$ : $\operatorname{dist}(x, \Gamma)>\varepsilon\}$. By the restrictions mentioned, $\Omega^{\varepsilon} \subset \Omega_{+}^{\varepsilon}$ and $\Omega_{-}^{\varepsilon} \subset \Omega \cap \Omega^{\varepsilon}$. Putting $\rho(x)=\operatorname{dist}\left(x, \partial \Omega_{+}^{2 \varepsilon}\right)$, we write the Hardy inequality

$$
\int_{\Omega_{+}^{2 \varepsilon}} \rho^{-4}|v(x)|^{2} d x \leq c_{1} \int_{\Omega_{+}^{2 \varepsilon}} \rho^{-2}\left|\nabla_{x} v(x)\right|^{2} d x \leq c_{2} \int_{\Omega_{+}^{2 \varepsilon}}\left|\nabla_{x}^{2} v(x)\right|^{2} d x,
$$

valid for functions of class $C_{c}^{\infty}\left(\Omega_{+}^{2 \varepsilon}\right)$ with constants $c_{1}$ and $c_{2}$ independent of $\varepsilon$. For a subdomain $\Xi \subset \Omega_{+}^{2 \varepsilon}$ we introduce the weighted norm

$$
\|v ; \Xi\|_{\beta}=\left(\int_{\Xi} \rho(x)^{\beta}\left|\nabla_{x}^{2} v(x)\right|^{2} d x\right)^{1 / 2} .
$$

The next statement can be proved, essentially, like Lemma 1 in 7 .

Lemma 4.2. For any $w \in \stackrel{\circ}{H}^{1}\left(\Omega_{+}^{\varepsilon}\right)$, we have

$$
\int_{\Omega_{+}^{\varepsilon} \backslash \Omega_{-}^{\varepsilon}}|w(x)|^{2} d x \leq c \varepsilon^{2} \int_{\Omega_{+}^{\varepsilon} \backslash \Omega_{-}^{\varepsilon}}\left|\nabla_{x} w(x)\right|^{2} d x
$$

and

$$
\int_{\partial \Omega_{-}^{\varepsilon}}|w(x)|^{2} d x \leq c \varepsilon \int_{\Omega_{+}^{\varepsilon} \backslash \Omega_{-}^{\varepsilon}}\left|\nabla_{x} w(x)\right|^{2} d x .
$$

Here $c$ is a constant depending on $\Omega$.

Corollary 4.1. 1) Let $w \in H\left(\right.$ or $\left.w \in H^{\varepsilon}\right)$ be orthogonal to $H \cap H^{\varepsilon}$ with respect to the scalar product $\langle\rangle,\left(\right.$ or $\left.\langle,\rangle_{\varepsilon}\right)$. Then

$$
\begin{aligned}
& \int_{\Omega}|w(x)|^{2} d x \leq c_{\beta} \varepsilon^{1+\beta} \int_{\Omega} \rho(x)^{-\beta}\left|\nabla_{x}^{2} w(x)\right|^{2} d x \\
& \left(\text { or } \int_{\Omega^{\varepsilon}}|w(x)|^{2} d x \leq c_{\beta} \varepsilon^{1+\beta} \int_{\Omega^{\varepsilon}} \rho(x)^{-\beta}\left|\nabla_{x}^{2} w(x)\right|^{2} d x\right) .
\end{aligned}
$$

2) The factor $\nu$ in inequality (4.1) does not exceed $c \varepsilon$.

Proof. Applying estimate (4.13) to the gradient $\nabla_{x} w$, we get

$$
\int_{\Omega_{+}^{\varepsilon} \backslash \Omega_{-}^{\varepsilon}}\left|\nabla_{x} w(x)\right|^{2} d x \leq c \varepsilon^{2} \int_{\Omega_{+}^{\varepsilon} \backslash \Omega_{-}^{\varepsilon}}\left|\nabla_{x}^{2} w(x)\right|^{2} d x .
$$

Similarly, inequality (4.14) shows that

$$
\int_{\Omega_{-}^{\varepsilon}}\left|\nabla_{x} w(x)\right|^{2} d x \leq C \varepsilon \int_{\Omega_{+}^{\varepsilon} \backslash \Omega_{-}^{\varepsilon}}\left|\nabla_{x}^{2} w(x)\right|^{2} d x .
$$

Using Theorem $D(p)$ in [55, we find

$$
\int_{\Omega_{-}^{\varepsilon}}\left|\nabla_{x} w(x)\right|^{2} d x \leq C \int_{\partial \Omega_{-}^{\varepsilon}}\left(\mid \nabla_{x} w\left(\left.(x)\right|^{2}+|w(x)|^{2}\right) d x .\right.
$$

Now relations (4.16) and (4.17)) lead to the estimate

$$
\int_{\Omega_{+}^{\varepsilon}}\left|\nabla_{x} w(x)\right|^{2} d x \leq C \varepsilon \int_{\Omega_{+}^{\varepsilon} \backslash \Omega_{-}^{\varepsilon}}\left|\nabla_{x}^{2} w(x)\right|^{2} d x,
$$

which ensures inequality (4.15), because $0<c \leq \rho(x) / \varepsilon \leq C$ for $x \in \Omega_{+}^{\varepsilon} \backslash \Omega_{-}^{\varepsilon}$.

The second claim of the lemma follows from the first for $\beta=0$. 
Lemma 4.3. Suppose $\varphi \in \mathfrak{X}_{p}$ and $\left\|\varphi ; H^{\varepsilon}\right\|=1$.

1) We have

$$
\int_{\Omega_{\backslash} \Omega_{-}^{2 \varepsilon}}\left|\nabla_{x}^{k} \varphi(x)\right|^{2} d x \leq c \varepsilon^{5-2 k}, \quad k=0,1,2, \quad \int_{\Gamma}\left|\nabla_{x}^{2} \varphi(x)\right|^{2} d s_{x} \leq c .
$$

2) The function $\Phi_{\varphi}^{\varepsilon}$ determined by $\varphi$ in accordance with (1.21) satisfies the estimate

$$
\int_{\Omega^{\varepsilon}}\left|\nabla_{x}^{k} \Phi_{\varphi}^{\varepsilon}(x)\right|^{2} d x \leq c \varepsilon^{3-k}, \quad k=1,2 .
$$

Proof. 1) We represent $\varphi$ as the sum $\phi_{1}+\phi_{2}$, where $\phi_{1} \in H^{6}\left(\mathbb{B}_{R}^{d}\right)$ is the solution of the problem

$$
\Delta_{x}^{2} \phi_{1}(x)=\lambda_{p} \varphi(x), \quad x \in \mathbb{B}_{R}^{d}, \quad \phi_{1}(x)=\partial_{|x|} \phi_{1}(x)=0, \quad x \in \partial \mathbb{B}_{R}^{d} .
$$

Here $d \geq 2$ is the dimension, $\mathbb{B}_{R}^{d}$ is a ball with large radius $R$ (it includes $\Omega^{2 \varepsilon}$ ), and $H^{l}$ is the Sobolev class. The second summand $\phi_{2}$ is the solution of the boundary-value problem

$$
\Delta_{x}^{2} \phi_{2}(x)=0, \quad x \in \Omega, \quad \phi_{2}(x)=-\phi_{1}(x), \quad \partial_{n} \phi_{2}(x)=-\partial_{n} \phi_{1}(x), \quad x \in \Gamma .
$$

Put $\Gamma_{\ell}=\{x \in \Omega: \operatorname{dist}(x, \Gamma)=\ell\}$. By the trace inequality, we have

$$
\int_{\Gamma_{\ell}}\left(\left|\phi_{1}(x)\right|^{2}+\left|\nabla_{x} \phi_{1}(x)\right|^{2}+\left|\nabla_{x}^{2} \phi_{1}(x)\right|^{2}\right) d s_{x} \leq c .
$$

Applying this estimate with $\ell=0$, and also Theorems $D(p)$ and $R(p)$ in [55] to $\phi_{2}$, we see that

$$
\int_{\Gamma_{\ell}}\left(\left|\nabla_{x} \phi_{2}(x)\right|^{2}+\left|\nabla_{x}^{2} \phi_{2}(x)\right|^{2}\right) d s_{x} \leq c .
$$

This relation with $\ell=0$ ensures the second inequality in (4.18). On the other hand, integration of the same relations over the parameter $\ell$ results in the first inequality in (4.18) for $k=2$, and for $k=0,1$ the required inequality follows from (4.13).

2) Let $\mathfrak{x}(x)$ be a smooth function equal to 1 outside of $\Omega_{-}^{\varepsilon}$ and to zero in $\Omega_{-}^{2 \varepsilon}$. We may assume that this function satisfies the conditions $\left|\nabla_{x}^{k} \mathfrak{x}(x)\right| \leq c \varepsilon^{-k}$ for $k=0,1,2$. We represent $\Phi_{\varphi}^{\varepsilon}$ in the form $\Psi_{\varphi}^{\varepsilon}-\mathfrak{x} \varphi$. Then $\Psi_{\varphi}^{\varepsilon}$ must solve the boundary-value problem

$$
\Delta_{x}^{2} \Psi_{\varphi}^{\varepsilon}(x)=\Delta_{x}^{2}(\mathfrak{x}(x) \varphi(x)), \quad x \in \Omega^{\varepsilon}, \quad \Psi_{\varphi}^{\varepsilon}(x)=\partial_{n} \Psi_{\varphi}^{\varepsilon}(x)=0, \quad x \in \Gamma^{\varepsilon} .
$$

We have

$$
\begin{aligned}
\left\langle\Psi_{\varphi}^{\varepsilon},\right. & \left.\Psi_{\varphi}^{\varepsilon}\right\rangle_{\varepsilon}=\int_{\Omega^{\varepsilon}}\left(\varphi(x) \Delta_{x} \mathfrak{x}(x)+2 \nabla_{x} \mathfrak{x}(x) \cdot \nabla_{x} \varphi(x)\right) \Delta_{x} \Psi_{\varphi}^{\varepsilon}(x) d x \\
& \quad+\int_{\Omega^{\varepsilon}} \Delta_{x} \varphi(x)\left(\Delta_{x}\left(\mathfrak{x}(x) \Psi_{\varphi}^{\varepsilon}(x)\right)-2 \nabla_{x} \mathfrak{x}(x) \cdot \nabla_{x} \Psi_{\varphi}^{\varepsilon}(x)-\Delta_{x} \mathfrak{x}(x) \Psi_{\varphi}^{\varepsilon}(x)\right) d x \\
= & \int_{\Omega^{\varepsilon}}\left(\varphi(x) \Delta_{x} \mathfrak{x}(x)+2 \nabla_{x} \mathfrak{x}(x) \cdot \nabla_{x} \varphi(x)\right) \Delta_{x} \Psi_{\varphi}^{\varepsilon}(x) d x \\
& -\int_{\Omega^{\varepsilon}}\left(-\lambda_{p} \varphi(x) \mathfrak{x}(x) \Psi_{\varphi}^{\varepsilon}(x)+\Delta_{x} \varphi(x)\left(2 \nabla_{x} \mathfrak{x}(x) \cdot \nabla_{x} \Psi_{\varphi}^{\varepsilon}(x)+\Delta_{x} \mathfrak{x}(x) \Psi_{\varphi}^{\varepsilon}(x)\right)\right) d x
\end{aligned}
$$


Consequently,

$$
\begin{aligned}
\left\|\Psi_{\varphi}^{\varepsilon} ; H_{\varphi}^{\varepsilon}\right\|^{2} \leq c & \left(\varepsilon^{-2}\left\|\varphi ; L_{2}\left(\Omega^{\varepsilon} \backslash \Omega_{-}^{2 \varepsilon}\right)\right\|\left\|\nabla_{x}^{2} \Psi_{\varphi}^{\varepsilon} ; L_{2}\left(\Omega^{\varepsilon} \backslash \Omega_{-}^{2 \varepsilon}\right)\right\|\right. \\
& +\varepsilon^{-1}\left\|\nabla_{x} \varphi ; L_{2}\left(\Omega \backslash \Omega_{-}^{2 \varepsilon}\right)\right\|\left\|\nabla_{x}^{2} \Psi_{\varphi}^{\varepsilon} ; L_{2}\left(\Omega^{\varepsilon} \backslash \Omega_{-}^{2 \varepsilon}\right)\right\| \\
& +\varepsilon^{-1}\left\|\nabla_{x}^{2} \varphi ; L_{2}\left(\Omega \backslash \Omega_{-}^{2 \varepsilon}\right)\right\|\left\|\nabla_{x} \Psi_{\varphi}^{\varepsilon} ; L_{2}\left(\Omega^{\varepsilon} \backslash \Omega_{-}^{2 \varepsilon}\right)\right\| \\
& +\varepsilon^{-2}\left\|\nabla_{x}^{2} \varphi ; L_{2}\left(\Omega \backslash \Omega_{-}^{2 \varepsilon}\right)\right\|\left\|\Psi_{\varphi}^{\varepsilon} ; L_{2}\left(\Omega^{\varepsilon} \backslash \Omega_{-}^{2 \varepsilon}\right)\right\| \\
& \left.+\left\|\varphi ; L_{2}\left(\Omega \backslash \Omega_{-}^{2 \varepsilon}\right)\right\|\left\|\Psi_{\varphi}^{\varepsilon} ; L^{2}\left(\Omega^{\varepsilon} \backslash \Omega_{-}^{2 \varepsilon}\right)\right\|\right) .
\end{aligned}
$$

Using estimate (4.13) for the function $\Psi_{\varphi}^{\varepsilon}$, we see that

$$
\begin{aligned}
\left\|\Psi_{\varphi}^{\varepsilon} ; H^{\varepsilon}\right\| \leq c & \left(\varepsilon^{-2}\left\|\varphi ; L_{2}\left(\Omega \backslash \Omega_{-}^{2 \varepsilon}\right)\right\|\right. \\
& \left.+\varepsilon^{-1}\left\|\nabla_{x} \varphi ; L^{2}\left(\Omega \backslash \Omega_{-}^{2 \varepsilon}\right)\right\|+\left\|\nabla_{x}^{2} \varphi ; L_{2}\left(\Omega \backslash \Omega_{-}^{2 \varepsilon}\right)\right\|\right) .
\end{aligned}
$$

Combining this with (4.18), we deduce the relation

$$
\left\|\Psi_{\varphi}^{\varepsilon} ; H^{\varepsilon}\right\|^{2} \leq c \varepsilon
$$

which ensures (4.19) for $k=2$. Also, Lemma 4.2 shows that

$$
\int_{\Omega^{\varepsilon} \backslash \Omega_{-}^{2 \varepsilon}}\left|\Psi_{\varphi}^{\varepsilon}(x)\right|^{2} d x \leq c \varepsilon^{5}, \quad \int_{\Omega^{\varepsilon} \backslash \Omega_{-}^{2 \varepsilon}}\left|\nabla_{x} \Psi_{\varphi}^{\varepsilon}(x)\right|^{2} d x \leq c \varepsilon^{3}
$$

and

$$
\int_{\partial \Omega_{-}^{2 \varepsilon}}\left|\Psi_{\varphi}^{\varepsilon}(x)\right|^{2} d x \leq c \varepsilon^{4}, \quad \int_{\partial \Omega_{-}^{2 \varepsilon}}\left|\nabla_{x} \Psi_{\varphi}^{\varepsilon}(x)\right|^{2} d x \leq c \varepsilon^{2} .
$$

By Theorem $D(p)$ in [55] applied to $\Psi_{\varphi}^{\varepsilon}$ in the domain $\Omega_{-}^{2 \varepsilon}$, and by (4.21), we obtain

$$
\left\|\nabla_{x} \Psi_{\varphi}^{\varepsilon}(x) ; L_{2}\left(\Omega_{-}^{2 \varepsilon}\right)\right\|^{2} d x \leq c \varepsilon^{2} .
$$

Together with (4.20), this inequality implies (4.19) with $k=1$.

The next statement is a consequence of (4.18), (4.19), and (4.11).

Corollary 4.2. We have

$$
\langle\mathfrak{B} \varphi, \mathfrak{S} \psi\rangle_{\varepsilon}=-\lambda_{p}{ }^{-1}\left(\left\langle\Phi_{\varphi}^{\varepsilon}, \Phi_{\psi}^{\varepsilon}\right\rangle_{\varepsilon}+\left(\Delta_{x} \varphi, \Delta_{x} \psi\right)_{\Omega \backslash \Omega^{\varepsilon}}\right)+O\left(\varepsilon^{2}\right) .
$$

In a domain $\Xi$ such that $\Omega_{-}^{\varepsilon} \subset \Xi \subset \Omega_{+}^{\varepsilon}$, we consider the following boundary-value problem for $u \in \stackrel{\circ}{H}^{2}(\Xi)$ :

$$
\left(\Delta_{x} u, \Delta_{x} w\right)_{\Xi}=\sum_{|\alpha| \leq 2}\left(f_{\alpha}, \partial_{x}^{\alpha} w\right)_{\Xi}, \quad w \in \stackrel{\circ}{H}^{2}(\Xi) ;
$$

here $\alpha=\left(\alpha_{1}, \ldots, \alpha_{d}\right)$ is multi-index, $\partial_{x}^{\alpha}=\partial^{|\alpha|} / \partial x_{1}^{\alpha_{1}} \cdots \partial x_{d}^{\alpha_{d}}$, and $f_{\alpha} \in L_{2}(\Xi)$. Also, we introduce the regularized distance $r_{\varepsilon}$ to the boundary of $\Omega_{-}^{2 \varepsilon}$. This function satisfies $\left|\nabla_{x}^{k} r_{\varepsilon}(x)\right| \leq c_{k} \rho^{1-k}, k=0,1,2, \ldots$, and $r_{\varepsilon}(x) \geq c \rho$, where $c>0$ and $\rho$ is the quantity introduced before formula (4.12).

Lemma 4.4. There exists a number $\beta_{0} \in(0,1)$ such that for $\beta \in\left(-\beta_{0}, \beta_{0}\right)$ the solution $u \in \stackrel{\circ}{H}^{2}(\Xi)$ of problem (4.23) satisfies the weighted estimate

$$
\int_{\Xi} \rho^{\beta}\left|\nabla_{x}^{2} u(x)\right|^{2} d x \leq c \sum_{|\alpha| \leq 2} \int_{\Xi} \rho^{\beta+4-2|\alpha|}\left|f_{\alpha}(x)\right|^{2} d x,
$$

where $c$ depends only on $\beta_{0}$ and the domain $\Xi$. 
Proof. We put $w=r_{\varepsilon}{ }^{\beta} u$ and $v=r_{\varepsilon}{ }^{\beta / 2} u$ in the integral identity (4.23). We have

$$
\left(\Delta_{x}\left(r_{\varepsilon}{ }^{-\beta / 2} v\right), \Delta_{x}\left(r_{\varepsilon}^{\beta / 2} v\right)\right)_{\Xi}=\sum_{|\alpha| \leq 2}\left(f_{\alpha}, \partial^{\alpha}\left(r_{\varepsilon}^{\beta / 2} v\right)\right)_{\Xi}
$$

The left-hand side is equal to the scalar product

$$
\begin{aligned}
\left(\Delta_{x} v\right. & -\frac{\beta}{r_{\varepsilon}} \nabla_{x} r_{\varepsilon} \cdot \nabla_{x} v+\frac{\beta(\beta+2)}{4 r_{\varepsilon}^{2}}\left|\nabla_{x} r_{\varepsilon}\right|^{2} v \\
& \left.\left.-\frac{\beta \Delta_{x} r_{\varepsilon}}{2 r_{\varepsilon}} v, \Delta_{x} v+\frac{\beta}{r_{\varepsilon}} \nabla r_{\varepsilon} \cdot \nabla v+\frac{\beta(\beta-2)}{4 r_{\varepsilon}^{2}}\left|\nabla r_{\varepsilon}\right|^{2} v+\frac{\beta \Delta r_{\varepsilon}}{2 r_{\varepsilon}} v\right)\right)_{\Xi},
\end{aligned}
$$

and hence, to the sum

$$
\begin{aligned}
\left(\Delta_{x} v, \Delta_{x} v\right)_{\Xi} & +\left(\Delta_{x} v, \frac{\beta^{2}}{2 r_{\varepsilon}^{2}}\left|\nabla_{x} r_{\varepsilon}\right|^{2} v\right)_{\Xi} \\
& -\frac{\beta^{2}}{4}\left(\nabla r_{\varepsilon} \cdot \nabla v, \nabla r_{\varepsilon} \cdot \nabla v\right)_{\Xi}+\beta^{2}\left(r_{\varepsilon}{ }^{-1} \nabla r_{\varepsilon} \cdot \nabla v, \frac{\left|\nabla r_{\varepsilon}\right|^{2}}{\left.r_{\varepsilon}^{2} v\right)_{\Xi}}\right. \\
& -\beta^{2}\left(\frac{1}{r_{\varepsilon}} \nabla_{x} r_{\varepsilon} \cdot \nabla_{x} v, \frac{\Delta r_{\varepsilon}}{2 r_{\varepsilon}} v\right)_{\Xi}+\left(\frac{\beta(\beta+2)}{4 r_{\varepsilon}{ }^{2}}\left|\nabla_{x} r_{\varepsilon}\right|^{2} v, \frac{\beta(\beta-2)}{4 r_{\varepsilon}{ }^{2}}\left|\nabla_{x} r_{\varepsilon}\right|^{2} v\right)_{\Xi} \\
& +\beta^{2}\left(\frac{1}{r_{\varepsilon}^{2}}\left|\nabla_{x} r_{\varepsilon}\right|^{2} v, \frac{\Delta r_{\varepsilon}}{2 r_{\varepsilon}} v\right)_{\Xi}-\beta^{2}\left(\frac{\Delta_{x} r_{\varepsilon}}{2 r_{\varepsilon}} v, \frac{\Delta r_{\varepsilon}}{2 r_{\varepsilon}} v\right)_{\Xi} .
\end{aligned}
$$

Using the Hardy inequalities (4.12), we obtain

$$
\left(\Delta_{x}\left(r_{\varepsilon}^{-\beta / 2} v\right), \Delta_{x}\left(r_{\varepsilon}^{\beta / 2} v\right)\right)_{\Xi} \geq\left(1-c \beta^{2}\right)\left(\Delta_{x} v, \Delta_{x} v\right)_{\Xi} .
$$

The same inequalities allow us to estimate the right-hand side of (4.25) as follows:

$$
\sum_{|\alpha| \leq 2}\left(f_{\alpha}, \nabla_{x}^{\alpha}\left(r_{\varepsilon}^{\beta / 2} v\right)\right)_{\Xi} \leq c \sum_{|\alpha| \leq 2}\left\|r_{\varepsilon}^{\beta / 2+2-|\alpha|} f_{\alpha} ; L_{2}(\Xi)\right\|\left\|\Delta_{x} v ; L_{2}(\Xi)\right\| .
$$

The last two inequalities yield estimate (4.24) for small $\beta$.

Applying Lemma 4.4 to the operators $\mathfrak{S}, \mathfrak{S}^{*}$ and to the projection to the subspace $H_{1} \cap H_{2}$, we obtain several important formulas.

Corollary 4.3. There exists $\beta_{0} \in(0,1)$ such that for $|\beta| \leq \beta_{0}$ the following statements are valid.

1) If $v \in H^{\varepsilon}$, then $\left\|\mathfrak{S}^{*} v ; \Omega\right\|_{\beta} \leq c\left\|v ; \Omega^{\varepsilon}\right\|_{\beta}$.

2) If $v \in H$, then $\left\|\mathfrak{S} v ; \Omega^{\varepsilon}\right\|_{\beta} \leq c\|v ; \Omega\|_{\beta}$.

3) Let $P$ be the projection from $\stackrel{\circ}{H}^{2}\left(\Omega \cup \Omega_{\varepsilon}\right)$ onto $\stackrel{\circ}{H}^{2}\left(\Omega \cap \Omega_{\varepsilon}\right)$ orthogonal with respect to the scalar product $\langle$,$\rangle . If v \in \stackrel{\circ}{H}^{2}\left(\Omega \cup \Omega_{\varepsilon}\right)$, then

$$
\left\|P v ; \Omega \cap \Omega_{\varepsilon}\right\|_{\beta} \leq C\left\|v ; \Omega \cup \Omega_{\varepsilon}\right\|_{\beta} .
$$

Proof. Since $\left\langle\mathfrak{S}^{*} v, w\right\rangle=\langle v, w\rangle$ for $w \in H$, Lemma 4.4 ensures statement 1). The second statement is also checked with the help of the definition of the operator $\mathfrak{S}$.

The operator $P$ is given by the formulas $u=P v$ and

$$
(\Delta u, \Delta w)_{\Omega \cap \Omega^{\varepsilon}}=(\Delta v, \Delta w)_{\Omega \cap \Omega^{\varepsilon}}, \quad w \in \stackrel{\circ}{H}^{2}\left(\Omega \cap \Omega^{\varepsilon}\right) .
$$

Therefore, statement 3 follows from Lemma 4.4. 
5. Proof of the asymptotic formula of Subsection 3 in $\S 1$. We check relation (1.19). For this, we transform the scalar product $\left\langle\mathfrak{b}_{\varphi}, \mathfrak{B} \psi\right\rangle_{\varepsilon}$ (see Subsection 3 in $\S 4$ for the definitions). We write $\mathfrak{b}_{\varphi}$ as the sum $\lambda_{p} \mathfrak{Q}_{p} \mathfrak{B} \varphi+\mathfrak{d}_{\varphi}$, where $\mathfrak{d}_{\varphi}$ is defined by the formula

$$
\lambda_{p}^{-1} \mathfrak{d}_{\varphi}-\mathfrak{Q}_{p} K^{\varepsilon} \mathfrak{d}_{\varphi}=g, \quad g=\lambda_{p} \mathfrak{Q}_{p} K^{\varepsilon} \mathfrak{Q}_{p} \mathfrak{B} \varphi .
$$

This formula (4.26) is completely similar to equation (27) in 7], i.e., by estimate (33) in the same paper, the solution of our equation satisfies $\left\|\mathfrak{d}_{\varphi} ; \stackrel{\circ}{H}^{2}\left(\Omega^{\varepsilon}\right)\right\| \leq c\left\|g ; \stackrel{\circ}{H}^{2}\left(\Omega^{\varepsilon}\right)\right\|$. Since the operators $\mathfrak{Q}_{p}$ and $K^{\varepsilon}$ are bounded in the space $H^{\varepsilon}$, formula (4.6) with the replacement $\nu \mapsto c \varepsilon$ (see Corollary 4.1 2)) shows that

$$
\left\|\mathfrak{d}_{\varphi} ; \stackrel{\circ}{H}^{2}\left(\Omega^{\varepsilon}\right)\right\| \leq c \varepsilon^{1 / 2}\left\|\varphi ; \stackrel{\circ}{H}^{2}\left(\Omega^{\varepsilon}\right)\right\| .
$$

We rewrite (4.26) as follows:

$$
\left\langle\mathfrak{d}_{\varphi}, w\right\rangle_{\varepsilon}=\lambda_{p}\left\langle K^{\varepsilon} \mathfrak{d}_{\varphi}, \mathfrak{Q}_{p} w\right\rangle_{\varepsilon}+\lambda_{p}\langle g, w\rangle_{\varepsilon}, \quad w \in H^{\varepsilon} .
$$

The right-hand side can be reshaped to

$$
\lambda_{p}\left(\mathfrak{d}_{\varphi}, \mathfrak{Q}_{p} w\right)_{\Omega^{\varepsilon}}+\lambda_{p}^{2}\left(\mathfrak{Q}_{p} \mathfrak{B} \varphi, \mathfrak{Q}_{p} w\right)_{\Omega^{\varepsilon}} .
$$

We want to rewrite this expression as the scalar product $(F, w)_{\Omega^{\varepsilon}}$. For this, we fix an orthonormal basis $\left\{\mathfrak{f}_{i}\right\}_{i=0}^{\varkappa_{p}-1}$ in $\mathfrak{S} \mathfrak{X}_{p}$ and expand the operator $\mathfrak{P}_{p}$ introduced before Lemma 4.1 with respect to this basis:

$$
\mathfrak{P}_{p} v=\sum_{i=0}^{\varkappa_{p}-1}\left\langle v, \mathfrak{f}_{i}\right\rangle_{\varepsilon} \mathfrak{f}_{i} .
$$

Thus,

$$
\left\langle\mathfrak{P}_{p} v, w\right\rangle_{\varepsilon}=\lambda_{p} \sum_{i=0}^{\varkappa_{p}-1}\left\langle v, \mathfrak{f}_{i}\right\rangle_{\varepsilon}\left(\mathfrak{f}_{i}, w\right)_{\Omega^{\varepsilon}} .
$$

As a result, expression (4.29) takes the form $(F, w)_{\Omega^{\varepsilon}}$ if we put

$$
F=\lambda_{p} \mathfrak{d}_{\varphi}+\lambda_{p}^{2} \mathfrak{Q}_{p} \mathfrak{B} \varphi-\lambda_{p}^{2} \sum_{i=0}^{\varkappa_{p}-1}\left\langle\mathfrak{d}_{\varphi}+\lambda_{p} \mathfrak{B} \varphi, \mathfrak{f}_{i}\right\rangle_{\varepsilon} \mathfrak{f}_{i} .
$$

Using (4.6) and (4.27), we obtain

$$
\left\|F ; L_{2}\left(\Omega^{\varepsilon}\right)\right\| \leq c\left(\left\|\mathfrak{d}_{\varphi} ; H^{\varepsilon}\right\|+\left\|\mathfrak{B} \varphi ; H^{\varepsilon}\right\|\right) \leq c \varepsilon^{1 / 2}\|\varphi ; H\| .
$$

Lemma 4.4 applied to problem (4.28) shows that for any $\beta \in\left(-\beta_{0}, \beta_{0}\right)$ we have

$$
\left(\int_{\Omega^{\varepsilon}} \rho^{\beta}\left|\nabla_{x}^{2} \mathfrak{d}_{\varphi}(x)\right|^{2} d x\right)^{1 / 2} \leq c \varepsilon^{1 / 2}\|\varphi ; H\| .
$$

Since $\Omega$ is a Lipschitz domain and $\psi \in \mathfrak{X}_{p}$, we can apply Theorem $R(p)$ in 55] to show that $\psi \in H_{\mathfrak{q}}^{2}(\Omega)$ with some $\mathfrak{q}>2$. Thus,

$$
\int_{\Omega} \rho^{-\beta}\left|\nabla_{x} \psi(x)\right|^{2} d x \leq c\|\psi ; H\|^{2}
$$

for some $\beta>0$. Relation (4.7) implies that

$$
\begin{aligned}
\left|\langle\mathfrak{B} \varphi, w\rangle_{\varepsilon}\right| \leq c\left(\|\varphi ; H\|\left\|w_{1} ; L_{2}\left(\Omega_{\varepsilon}\right)\right\|+\left\|\varphi_{1} ; L_{2}\left(\Omega_{\varepsilon}\right)\right\|\|w ; H\|\right. \\
\left.+\left\|\mathfrak{S} \varphi_{1} ; H\right\|\left\|w_{1} ; H^{\varepsilon}\right\|+\|\varphi ; H\|\left\|\mathfrak{S}^{*} w_{1} ; L_{2}\left(\Omega_{\varepsilon}\right)\right\|\right) .
\end{aligned}
$$


Applying Corollary 4.1, 1) to the functions $w_{1}, \varphi_{1}$ and $\mathfrak{S} \varphi_{1}, \mathfrak{S} w_{1}$, we obtain the estimate

$$
\begin{aligned}
\left|\langle\mathfrak{B} \varphi, w\rangle_{\varepsilon}\right| \leq C_{1} \varepsilon^{(1+\beta) / 2}\left(\left\|w_{1} ; \Omega_{\varepsilon}\right\|_{-\beta}\right. & +\left\|\varphi_{1} ; \Omega\right\|_{-\beta} \\
& \left.+\left\|\mathfrak{S} \varphi_{1} ; \Omega_{\varepsilon}\right\|_{-\beta}+\left\|\mathfrak{S}^{*} w_{1} ; \Omega\right\|_{-\beta}\right),
\end{aligned}
$$

where $C_{1}=c\left(\|\varphi ; H\|+\left\|w ; H^{\varepsilon}\right\|\right)$. Now, formulas (4.32), (4.31) and Corollary 4.3 imply the relations

$$
\left|\left\langle\mathfrak{d}_{\varphi}, \mathfrak{B} \psi\right\rangle_{\varepsilon}\right| \leq c \varepsilon^{1+\beta / 2}\|\varphi ; H\|\|\psi ; H\|, \quad\left|\langle\mathfrak{B} \varphi, \mathfrak{S} \psi\rangle_{\varepsilon}\right| \leq c \varepsilon^{(1+\beta) / 2}\|\varphi ; H\|\|\psi ; H\|
$$

for any $\varphi, \psi \in \mathfrak{X}_{p}$. In particular, it follows that, first, the quantity $\varsigma$ occurring in (4.2) does not exceed $c \varepsilon^{(1+\beta) / 2}$, and second, the asymptotic representation (4.3) can be written as

$$
\left(\lambda_{q}^{\varepsilon}\right)^{-1}=\lambda_{p}^{-1}+\varpi_{q}+O\left(\varepsilon^{1+\beta / 2}\right) .
$$

Here $\varpi_{p}, \ldots, \varpi_{p+\varkappa_{p}-1}$ are the eigenvalues of the following algebraic spectral problem for $\varphi \in \mathfrak{X}_{p}$ :

$$
\langle\mathfrak{B} \varphi, \mathfrak{S} \psi\rangle_{\varepsilon}+\lambda_{p}\left\langle\mathfrak{Q}_{p} \mathfrak{B} \varphi, \mathfrak{B} \psi\right\rangle_{\varepsilon}=\varpi\langle\mathfrak{S} \varphi, \mathfrak{S} \psi\rangle_{\varepsilon}, \quad \psi \in \mathfrak{X}_{p} .
$$

Next, using the representation (4.8) for $\mathfrak{S} \varphi \in \mathfrak{X}_{p}$ and Lemma 4.3, 1), we obtain

$$
\left\|\mathfrak{S} \varphi-\varphi ; H^{\varepsilon}\right\|^{2}=\left\|\Phi_{\varphi}^{\varepsilon} ; H^{\varepsilon}\right\|^{2} \leq c \varepsilon\|\varphi ; H\|^{2} .
$$

Relations (4.33) and (4.35) imply that $\varpi_{q}=O\left(\varepsilon^{(1+\beta) / 2}\right)$. Now, formula (4.30) shows that

$$
\left\langle\mathfrak{P}_{p} \mathfrak{B} \varphi, \mathfrak{B} \psi\right\rangle_{\varepsilon}=\sum_{q=p}^{p+\varkappa_{p}-1}\left\langle\mathfrak{B} \varphi, \mathfrak{S} \varphi_{q}\right\rangle_{\varepsilon}\left\langle\mathfrak{S} \varphi_{q}, \mathfrak{B} \psi\right\rangle_{\varepsilon}
$$

where $\mathfrak{S} \varphi_{q}=\mathfrak{f}_{q}$ and $\varphi_{q} \in \mathfrak{X}_{p}$. By (4.33), we have

$$
\left\langle\mathfrak{P}_{p} \mathfrak{B} \varphi, \mathfrak{B} \psi\right\rangle_{\varepsilon} \leq c \varepsilon^{1+\beta}\|\varphi ; H\|\|\psi ; H\| .
$$

The above observations allow us to write relations (4.34) in a shorter form:

$$
\left(\lambda_{q}^{\varepsilon}\right)^{-1}=\lambda_{p}^{-1}+\tau_{j}+O\left(\varepsilon^{1+\beta / 2}\right)
$$

here $\tau_{q}, \ldots, \tau_{p+\varkappa_{p}-1}$ are the eigenvalues of the algebraic spectral problem

$$
\langle\mathfrak{B} \varphi, \mathfrak{S} \psi\rangle_{\varepsilon}+\lambda_{p}\langle\mathfrak{B} \varphi, \mathfrak{B} \psi\rangle_{\varepsilon}=\tau\langle\varphi, \psi\rangle_{\varepsilon}, \quad \psi \in \mathfrak{X}_{p} .
$$

6. Auxiliary calculations. To simplify the expression $\langle\mathfrak{B} \varphi, \mathfrak{B} \psi\rangle_{\varepsilon}$, we write

$$
K^{\varepsilon} \varphi=\lambda_{p}^{-1} \mathfrak{S} \varphi-\lambda_{p}^{-1} \Psi_{\varphi}^{\varepsilon}
$$

here $\Psi_{\varphi}^{\varepsilon} \in H^{\varepsilon}$ is the solution of problem (1.22). Clearly, the function $\Psi_{\varphi}^{\varepsilon}$ is orthogonal to $H \cap H^{\varepsilon}$.

By (4.8) and (4.37), we get $\mathfrak{B} \varphi=K^{\varepsilon} \Phi_{\varphi}^{\varepsilon}-\lambda_{p}^{-1} \Psi_{\varphi}^{\varepsilon}$. Hence,

$$
\begin{aligned}
\langle\mathfrak{B} \varphi, \mathfrak{B} \psi\rangle_{\varepsilon}= & \lambda_{p}^{-2}\left\langle\Psi_{\varphi}^{\varepsilon}, \Psi_{\psi}^{\varepsilon}\right\rangle_{\varepsilon}+\left\langle K^{\varepsilon} \Phi_{\varphi}^{\varepsilon}, K^{\varepsilon} \Phi_{\psi}^{\varepsilon}\right\rangle_{\varepsilon} \\
& -\lambda_{p}^{-1}\left(\left\langle K^{\varepsilon} \Phi_{\varphi}^{\varepsilon}, \Psi_{\psi}^{\varepsilon}\right\rangle_{\varepsilon}+\left\langle K^{\varepsilon} \Psi_{\varphi}^{\varepsilon}, \Phi_{\psi}^{\varepsilon}\right\rangle_{\varepsilon}\right) .
\end{aligned}
$$

Lemma 4.5. We have

$$
\left\|\nabla_{x}^{2} \varphi_{\varphi}^{\varepsilon} ; L_{2}\left(\Omega \backslash \Omega^{\varepsilon}\right)\right\|^{2} \leq c \varepsilon^{\delta}, \quad\left\|\Psi_{\varphi}^{\varepsilon} ; L_{2}\left(\Omega^{\varepsilon}\right)\right\|^{2} \leq c \varepsilon^{1+\delta},
$$

where $\delta$ is a positive number depending on $\Omega$. 
Proof. Since $\nabla_{x}^{2} \varphi_{\varphi}^{\varepsilon} \in L_{q}(\Omega)$ with some $q>2$, the first inequality is valid. We check the second. For this, we put

$$
\Psi_{\varphi}^{\varepsilon}=\varphi-\Upsilon_{\varphi}^{\varepsilon},
$$

where $\Upsilon_{\varphi}^{\varepsilon} \in H^{\varepsilon}$ is the solution of the problem $\left\langle\Upsilon_{\varphi}^{\varepsilon}, w\right\rangle_{\varepsilon}=\lambda_{p}(\varphi, w)_{\Omega^{\varepsilon}}, w \in H^{\varepsilon}$.

We represent $\Upsilon_{\varphi}^{\varepsilon}$ as the sum $\Upsilon_{\varphi}^{\varepsilon 1}+\Upsilon_{\varphi}^{\varepsilon 2}$, where $\Upsilon_{\varphi}^{\varepsilon 1} \in \stackrel{\circ}{H}^{2}\left(\Omega_{+}^{\varepsilon}\right)$ satisfies $\Delta_{x}^{2} \Upsilon_{\varphi}^{\varepsilon 1}=\lambda_{p} \varphi$ in $\Omega_{+}^{\varepsilon}$, and $\Upsilon_{\varphi}^{\varepsilon 2}$ has the form $-\mathfrak{x} \Upsilon_{\varphi}^{\varepsilon 1}+\Theta_{\varphi}^{\varepsilon}$ with $\Theta_{\varphi}^{\varepsilon} \in \stackrel{\circ}{H}^{2}\left(\Omega^{\varepsilon}\right)$ satisfying the equation

$$
\Delta_{x}^{2} \Theta_{\varphi}^{\varepsilon}(x)=\Delta_{x}^{2}\left(\mathfrak{x}(x) \Upsilon_{\varphi}^{\varepsilon 1}(x)\right), \quad x \in \Omega^{\varepsilon}
$$

(the function $\mathfrak{x}$ was already defined in the course of the proof of Lemma 4.3).

Theorem $R(p)$ in [55] shows that $\Upsilon_{\varphi}^{\varepsilon 1} \in \stackrel{\circ}{H}_{q}^{2}\left(\Omega_{+}^{\varepsilon}\right)$ for some $q>2$, and that

$$
\left\|\Upsilon_{\varphi}^{\varepsilon 1} ; H_{q}^{2}\left(\Omega_{+}^{\varepsilon}\right)\right\| \leq c\left\|\varphi ; L^{2}(\Omega)\right\| .
$$

We multiply equation (4.41) by $\Theta_{\varphi}^{\varepsilon}$, integrate over $\Omega^{\varepsilon}$ and use the homogeneous boundary conditions, obtaining

$$
\int_{\Omega^{\varepsilon}}\left|\Delta_{x} \Theta_{\varphi}^{\varepsilon}(x)\right|^{2} d x=\int_{\Omega^{\varepsilon}} \Delta_{x}\left(\mathfrak{x}(x) \Upsilon_{\varphi}^{\varepsilon 1}(x)\right) \Delta_{x} \Theta_{\varphi}^{\varepsilon}(x) d x .
$$

Consequently,

$$
\begin{aligned}
\left\|\Delta_{x} \Theta_{\varphi}^{\varepsilon} ; L_{2}\left(\Omega^{\varepsilon}\right)\right\| \leq c & \left(\varepsilon^{-2}\left\|\Upsilon_{\varphi}^{\varepsilon 1} ; L_{2}\left(\Omega^{\varepsilon} \backslash \Omega_{-}^{2 \varepsilon}\right)\right\|\right. \\
& \left.+\varepsilon^{-1}\left\|\nabla_{x} \Upsilon_{\varphi}^{\varepsilon 1} ; L_{2}\left(\Omega^{\varepsilon} \backslash \Omega_{-}^{2 \varepsilon}\right)\right\|+\left\|\nabla_{x}^{2} \Upsilon_{\varphi}^{\varepsilon 1} ; L_{2}\left(\Omega^{\varepsilon} \backslash \Omega_{-}^{2 \varepsilon}\right)\right\|\right) .
\end{aligned}
$$

By (4.13) and (4.42), we have $\left\|\Delta_{x} \Theta_{\varphi}^{\varepsilon} ; L_{2}\left(\Omega^{\varepsilon}\right)\right\|^{2} \leq c \varepsilon^{1-2 / q}\left\|\varphi ; L_{2}(\Omega)\right\|^{2}$. The same formulas yield the relation

$$
\left\|\nabla_{x}^{2} \Upsilon_{\varphi}^{\varepsilon 1} ; L_{2}\left(\Omega^{\varepsilon} \backslash \Omega_{-}^{2 \varepsilon}\right)\right\|^{2}+\left\|\Delta_{x}\left(\mathfrak{x} \Upsilon_{\varphi}^{\varepsilon 1}\right) ; L_{2}\left(\Omega^{\varepsilon}\right)\right\|^{2} \leq c \varepsilon^{1-2 / q}\left\|\varphi ; L_{2}(\Omega)\right\|^{2}
$$

Therefore,

$$
\left\|\nabla_{x}^{2} \Upsilon_{\varphi}^{\varepsilon 2} ; L_{2}\left(\Omega^{\varepsilon} \backslash \Omega_{-}^{2 \varepsilon}\right)\right\|^{2} \leq c \varepsilon^{1-2 / q}\left\|\varphi ; L_{2}(\Omega)\right\|^{2} .
$$

The above estimates imply the inequality

$$
\int_{\Omega^{\varepsilon} \backslash \Omega_{-}^{2 \varepsilon}}\left|\nabla_{x}^{2} \Upsilon_{\varphi}^{\varepsilon}(x)\right|^{2} d x \leq c \varepsilon^{1-2 / q}\left\|\varphi ; L_{2}(\Omega)\right\|^{2} .
$$

Now Lemma 4.2 shows that

$$
\begin{aligned}
& \varepsilon^{-4}\left\|\Upsilon_{\varphi}^{\varepsilon} ; L_{2}\left(\Omega^{\varepsilon} \backslash \Omega_{-}^{2 \varepsilon}\right)\right\|^{2}+\varepsilon^{-1}\left\|\nabla_{x} \Upsilon_{\varphi}^{\varepsilon} ; L_{2}\left(\partial \Omega_{-}^{2 \varepsilon}\right)\right\|^{2}+\varepsilon^{-3}\left\|\Upsilon_{\varphi}^{\varepsilon} ; L_{2}\left(\partial \Omega_{-}^{2 \varepsilon}\right)\right\|^{2} \\
& \quad \leq c \varepsilon^{1-2 / q}\left\|\varphi ; L^{2}(\Omega)\right\|^{2} .
\end{aligned}
$$

By Theorem $D(p)$ in [55] and the formulas obtained, we have

$$
\left\|\nabla_{x} \Upsilon_{\varphi}^{\varepsilon} ; L_{2}\left(\Omega_{-}^{2 \varepsilon}\right)\right\|^{2} \leq c \varepsilon^{2-2 / q}\left\|\varphi ; L^{2}(\Omega)\right\|^{2} .
$$

So, taking (4.43) into account, we arrive at the required estimate (4.39).

Relations (4.39) and (4.8), (4.38) imply the identity

$$
\langle\mathfrak{B} \varphi, \mathfrak{B} \psi\rangle_{\varepsilon}=\lambda_{p}^{-2}\left\langle\Psi_{\varphi}^{\varepsilon}, \Psi_{\psi}^{\varepsilon}\right\rangle_{\varepsilon}+O\left(\varepsilon^{1+\delta}\right) ;
$$

combining this with (4.36), (4.39), and (4.22), we establish the desired asymptotic approximation (1.20). 
7. On the validity of the Hadamard formula. Suppose that the boundary of $\Omega$ is of class $C^{1, \alpha}, \alpha \in(0,1)$; then $\varphi$ falls into the Hölder space $C^{2, \alpha}(\Omega)$. In a neighborhood of the boundary $\Gamma$, with every point $x$ we associate a point $s \in \Gamma$ nearest to $x$ and a number $n$ such that $x=s+n \vec{n}(s)$; here $\vec{n}(s)$ is the outward unit normal to $\Gamma \ni s$. The boundary $\Gamma^{\varepsilon}$ is given by the equation $n=h^{\varepsilon}(s)$. Assume that $\left|h^{\varepsilon}(s)\right| \leq \varepsilon$ and that $\left|\nabla_{s} h^{\varepsilon}(s)\right|$ is bounded uniformly in $\varepsilon \in\left(0, \varepsilon_{0}\right]$ and satisfies condition (1.23). Our nearest goal is to check formula (1.24). Let the surface $\Gamma(\varepsilon)$ surrounding the domain $\Omega(\varepsilon)$ be defined by the equation $n=\varepsilon$. We write $\Upsilon_{\varphi}^{\varepsilon}$ as the sum $\Upsilon_{\varphi}^{\varepsilon 1}+\Upsilon_{\varphi}^{\varepsilon 2}$, where $\Upsilon_{\varphi}^{\varepsilon 1}$ solves the problem

$$
\Delta_{x}^{2} \Upsilon_{\varphi}^{\varepsilon 1}(x)=\lambda_{p} \varphi(x), \quad x \in \Omega(\varepsilon), \quad \Upsilon_{\varphi}^{\varepsilon 1}=\partial_{n} \Upsilon_{\varphi}^{\varepsilon 1}(x)=0, \quad x \in \Gamma(\varepsilon) .
$$

The function $\Upsilon_{\varphi}^{\varepsilon 2}$ satisfies the relations

$$
\Delta_{x} \Upsilon_{\varphi}^{\varepsilon 2}(x)=0, \quad x \in \Omega^{\varepsilon}, \quad \Upsilon_{\varphi}^{\varepsilon 2}(x)=-\Upsilon_{\varphi}^{\varepsilon 1}(x), \quad \partial_{n} \Upsilon_{\varphi}^{\varepsilon 2}(x)=-\partial_{n} \Upsilon_{\varphi}^{\varepsilon 1}(x), \quad x \in \Gamma^{\varepsilon} .
$$

Since $\Upsilon_{\varphi}^{\varepsilon 1} \in C^{2, \alpha}(\Omega(\varepsilon))$ and $\Upsilon_{\varphi}^{\varepsilon 1}=\partial_{n} \Upsilon_{\varphi}^{\varepsilon 1}=0$ on $\Gamma(\varepsilon)$, we have

$$
\begin{aligned}
& \left|\Upsilon_{\varphi}^{\varepsilon 1}(x)\right| \leq c\left|h^{\varepsilon}(s)-\varepsilon\right|^{2}, \quad\left|\nabla \Upsilon_{\varphi}^{\varepsilon 1}(x)\right| \leq c\left|h^{\varepsilon}(s)-\varepsilon\right|, \\
& \left|\nabla_{s} \nabla_{x} \Upsilon_{\varphi}^{\varepsilon 1}(x)\right| \leq c\left|h^{\varepsilon}(s)-\varepsilon\right|^{\alpha}, \quad x \in \Gamma^{\varepsilon},
\end{aligned}
$$

whence

$$
\begin{aligned}
& \int_{\Gamma^{\varepsilon}}\left|\Upsilon_{\varphi}^{\varepsilon 1}(x)\right|^{2} d s_{x}=O\left(\varepsilon^{4}\right), \quad \int_{\Gamma^{\varepsilon}}\left|\nabla_{x} \Upsilon_{\varphi}^{\varepsilon 1}(x)\right|^{2} d s_{x}=O\left(\varepsilon^{2}\right), \\
& \int_{\Gamma^{\varepsilon}}\left|\nabla_{x}^{2} \Upsilon_{\varphi}^{\varepsilon 1}(x)\right|^{2} d s_{x}=O(1) .
\end{aligned}
$$

Recalling the requirement (1.23), we see that the last inequality in (4.44) implies that

$$
\int_{\Gamma^{\varepsilon}}\left|\nabla_{s} \nabla_{x} \Upsilon_{\varphi}^{\varepsilon 1}\left(h^{\varepsilon}(s), s\right)\right|^{2} d s_{x}=o(1) \text {. }
$$

It follows that $\nabla_{x} \Upsilon_{\varphi}^{\varepsilon 2} \in H^{1 / 2}\left(\Gamma^{\varepsilon}\right)^{d}$ and $\left\|\nabla_{x} \Upsilon_{\varphi}^{\varepsilon 2} ; H^{1 / 2}\left(\Gamma^{\varepsilon}\right)\right\|=o(\varepsilon)$. Consequently, $\left\|\nabla_{x}^{2} \Upsilon_{\varphi}^{\varepsilon 2} ; L_{2}\left(\Omega^{\varepsilon}\right)\right\|^{2}=o(\varepsilon)$, whence $\left\langle\Psi_{\varphi}^{\varepsilon}, \Psi_{\psi}^{\varepsilon}\right\rangle_{\varepsilon}=\left\langle\varphi-\Upsilon_{\varphi}^{\varepsilon 1}, \psi-\Upsilon_{\psi}^{\varepsilon 1}\right\rangle_{\varepsilon}+o(\varepsilon)$.

Consider the function $\Theta_{\varphi}^{\varepsilon}=\varphi-\Upsilon_{\varphi}^{\varepsilon 1}$ in $\Omega \cap \Omega^{\varepsilon}$. It satisfies the equation $\Delta_{x}^{2} \Theta_{\varphi}^{\varepsilon}=0$ in $\Omega \cap \Omega^{\varepsilon}$ and the boundary conditions $\Theta_{\varphi}^{\varepsilon}=-\Upsilon_{\varphi}^{\varepsilon 1}, \partial_{n} \Theta_{\varphi}^{\varepsilon}=-\partial_{n} \Upsilon_{\varphi}^{\varepsilon 1}$ on the boundary $\Gamma_{0 \varepsilon}=\partial\left(\Omega \cap \Omega^{\varepsilon}\right)$. Since the curve $\Gamma_{0 \varepsilon}$ is defined by the relation $n=\min \left\{h^{\varepsilon}(z), 0\right\}$, with the help of (1.23) we find that formulas (4.45) and (4.46) remain valid if we replace $\Gamma^{\varepsilon}$ with $\Gamma_{0 \varepsilon}$.

Since $\varphi \in C^{2, \alpha}(\Omega)$, in the vicinity of $\Gamma$ we have

$$
|\varphi(x)| \leq c|n|^{2}, \quad\left|\nabla_{x} \varphi(x)\right| \leq c|n|, \quad\left|\nabla_{s} \nabla_{x} \varphi(x)\right| \leq c|n|^{\alpha}, \quad\left|\nabla_{x}^{2} \varphi(x)\right| \leq c .
$$

Combining this with (4.45) and (4.46), we see that

$$
\begin{aligned}
& \left\|\Theta_{\varphi}^{\varepsilon} ; L_{2}\left(\Gamma_{0 \varepsilon}\right)\right\|=O\left(\varepsilon^{2}\right), \quad\left\|\nabla_{x} \Theta_{\varphi}^{\varepsilon} ; L_{2}\left(\Gamma_{0 \varepsilon}\right)\right\|=O(\varepsilon), \\
& \left\|\nabla_{s} \nabla_{x} \Upsilon_{\varphi}^{\varepsilon 1} ; L_{2}\left(\Gamma_{0 \varepsilon}\right)\right\|=o(1) .
\end{aligned}
$$

Thus, $\nabla_{x} \Theta_{\varphi}^{\varepsilon} \in H^{1 / 2}\left(\Gamma_{0 \varepsilon}\right)^{d}$ and $\left\|\nabla_{x} \Theta_{\varphi}^{\varepsilon} ; H^{1 / 2}\left(\Gamma_{0 \varepsilon}\right)^{d}\right\|^{2}=o(\varepsilon)$, i.e., $\left\|\nabla_{x}^{2} \Theta_{\varphi}^{\varepsilon} ; L_{2}\left(\Omega \cap \Omega^{\varepsilon}\right)\right\|^{2}=$ $o(\varepsilon)$. Together with (4.40), the last relation shows that

$$
\left\langle\Psi_{\varphi}^{\varepsilon}, \Psi_{\psi}^{\varepsilon}\right\rangle_{\varepsilon}=\left(\Delta_{x} \Upsilon_{\varphi}^{\varepsilon 1}, \Delta_{x} \Upsilon_{\psi}^{\varepsilon 1}\right)_{\Omega^{\varepsilon} \backslash \Omega}+o(\varepsilon)
$$

Now, we can apply Theorem $R(p)$ in [55] to the function $\Theta_{\varphi}^{\varepsilon}$ and use (4.48) to check that $\left\|\nabla_{x}^{2} \Theta_{\varphi}^{\varepsilon} ; L_{2}\left(\Gamma_{0 \varepsilon}\right)\right\|=o(1)$, whence

$$
\left\|\Delta_{x} \Upsilon_{\varphi}^{\varepsilon 1} ; L_{2}\left(\Gamma_{0 \varepsilon}\right)\right\|^{2}=\left\|\Delta_{x} \varphi ; L_{2}\left(\Gamma_{0 \varepsilon}\right)\right\|^{2}+o(1) .
$$


Since $\Upsilon_{\varphi}^{\varepsilon 1} \in C^{2, \alpha}(\Omega(\varepsilon))$, we have

$$
\left|\nabla_{x}^{2} \Upsilon_{\varphi}^{\varepsilon 1}(n, s)-\nabla_{x}^{2} \Upsilon_{\varphi}^{\varepsilon 1}(0, s)\right| \leq c|n|^{\alpha} .
$$

Using also (4.50), we arrive at the identity

$$
\left(\Delta_{x} \Upsilon_{\varphi}^{\varepsilon 1}, \Delta_{x} \Upsilon_{\psi}^{\varepsilon 1}\right)_{\Omega^{\varepsilon} \backslash \Omega}=\int_{\Gamma \cap \Omega^{\varepsilon}} h(z) \Delta_{x} \varphi(x) \Delta_{x} \psi(x) d s_{x}+O\left(\varepsilon^{1+\alpha}\right) .
$$

With the help of (4.47) and (1.23), we can show that the function $\Phi_{\varphi}^{\varepsilon}$ satisfies estimate (4.48) with $\Theta_{\varphi}^{\varepsilon}$ and $\Gamma_{0 \varepsilon}$ in place of $\Phi_{\varphi}^{\varepsilon}$ and $\Gamma^{\varepsilon}$. For $\Phi_{\varphi}^{\varepsilon}$ we have $\left\|\nabla_{x} \Phi_{\varphi}^{\varepsilon} ; H^{1 / 2}\left(\Gamma^{\varepsilon}\right)\right\|^{2}=o(\varepsilon)$, whence

$$
\left\langle\Phi_{\varphi}^{\varepsilon}, \Phi_{\psi}^{\varepsilon}\right\rangle_{\varepsilon}=o(\varepsilon)
$$

Since $\varphi \in C^{2, \alpha}(\Omega)$, we obtain

$$
\left(\Delta_{x} \varphi, \Delta_{x} \psi\right)_{\Omega \backslash \Omega^{\varepsilon}}=\int_{\Gamma_{0 \varepsilon}}|h(s)| \Delta_{x} \varphi(x) \Delta_{x} \psi(x) d s_{x}+o(\varepsilon) .
$$

Now formulas (4.52), (4.53), (4.49), (4.51), and also (1.19) and (1.20), imply the required relations (1.24) and (1.25).

8. Plane domains with corner points. Suppose that the boundary $\Gamma$ of a domain $\Omega \subset \mathbb{R}^{2}$ has corner points $P_{1}, \ldots, P_{Q}$, but is smooth (of class $C^{1, \delta}$ with some $\delta \in(0,1)$ ) outside of these points. Let $\alpha_{q}$ denote the opening of the angle with the vertex $P_{q}$, measured inside of $\Omega, \alpha_{q}<2 \pi$. The results on solutions of the Dirichlet problem for elliptic equations (see Chapter 7 in [56]) show that there exist quantities $\Lambda_{q}>3 / 2$ such that

$$
\begin{aligned}
|\varphi(x)| & +r(x)\left|\nabla_{x} \varphi(x)\right|+r(x)^{2}\left|\nabla_{x}^{2} \varphi(x)\right| \\
& +|x-y|^{-\delta}\left(\mid r(x)^{2} \nabla_{x}^{2} \varphi(x)-r(y)^{2} \nabla_{y}^{2} \varphi(y)\right) \leq c R_{\Lambda},
\end{aligned}
$$

where $r=r_{1} r_{2} \cdots r_{l}, r_{q}(x)=\left|x-P_{q}\right|$, and $R_{\Lambda}=\prod_{q=1}^{l} r_{q}^{\Lambda_{q}}$.

Suppose $\Omega^{\varepsilon} \subset \Omega$. Then we have the asymptotic formula (1.19), but now the functions $\Psi_{\varphi}^{\varepsilon}$ and $\Psi_{\psi}^{\varepsilon}$ in (1.20) are equal to zero, so that the exponents $\sigma_{p}, \ldots, \sigma_{p+\varkappa_{p}-1}$ in (1.19) are the eigenvalues of the problem

$$
\lambda_{p}^{-1}\left(-\left(\Delta_{x} \Phi_{\varphi}, \Delta_{x} \Phi_{\psi}\right)_{\Omega^{\varepsilon}}-\left(\Delta_{x} \varphi, \Delta_{x} \psi\right)_{\Omega \backslash \Omega^{\varepsilon}}\right)=\sigma\left(\Delta_{x} \varphi, \Delta_{x} \psi\right)_{\Omega}, \quad \psi \in \mathfrak{X}_{p} .
$$

Let $\Gamma^{\prime}=\left\{s \in \Gamma:\left|s-p_{q}\right|>c \varepsilon\right\}$. In the vicinity of $\Gamma^{\prime}$ we use the local coordinates $n$, $s$ (see Subsection 1 in $\S 1$ ). The part of the curve $\Gamma_{\varepsilon}$ located "above" $\Gamma^{\prime}$ is given by the equation $n=h^{\varepsilon}(s)$; we denote this part by $\Gamma_{\varepsilon}^{\prime}$. Assume that $\left|h^{\varepsilon}(s)\right| \leq \varepsilon,\left|\partial_{s} h^{\varepsilon}(s)\right| \leq c$, and

$$
\int_{\Gamma_{\varepsilon}^{\prime}} R_{\Lambda}(x)^{2} r(x)^{-4}\left|\partial_{s} h^{\varepsilon}(s)\right|^{2} d s_{x}=o(1) .
$$

Also, we assume that the remaining part of $\Gamma^{\varepsilon}$ is located in the annuli $\mathbb{B}_{c_{2} \varepsilon}\left(P_{q}\right) \backslash \mathbb{B}_{c_{1} \varepsilon}\left(P_{q}\right)$, where $c_{1}<c_{2}$, and that each of such parts is Lipschitz with a constant bounded uniformly relative to $\varepsilon \in\left(0, \varepsilon_{0}\right]$.

Since $\varphi=\partial_{n} \varphi=0$ on $\Gamma=\partial \Omega$, estimate (4.54) yields

$$
\left|\partial_{s} \nabla_{x} \varphi(n, s)\right| \leq c|n|^{\delta} R_{\Lambda}(x) r(x)^{-2-\delta}, \quad\left|\nabla_{x}^{2} \varphi(s, n)\right| \leq c R_{\Lambda}(x) r(x)^{-2} .
$$

Consequently,

$$
\left|\partial_{s} \nabla_{x} \varphi\left(h^{\varepsilon}(s), s\right)\right| \leq c\left(|n|^{\delta} R_{\Lambda}(x) r(x)^{-2-\delta}+R_{\Lambda}(x) r(x)^{-2}\left|\partial_{s} h^{\varepsilon}(s)\right|\right) .
$$

Moreover, by (4.54) we have

$$
\left|\varphi\left(h^{\varepsilon}(s), s\right)\right| \leq \operatorname{ch}(\varepsilon, s)^{2} R_{\Lambda}(x) r(x)^{-2}, \quad\left|\nabla_{x} \varphi\left(h^{\varepsilon}(s), s\right)\right| \leq \operatorname{ch}^{\varepsilon}(s) R_{\Lambda}(x) r(x)^{-2},
$$


whence

$$
\int_{\Gamma_{\varepsilon}^{\prime}}\left|\varphi\left(h^{\varepsilon}(s), s\right)\right|^{2} d s_{x}=O\left(\varepsilon^{4}\right), \quad \int_{\Gamma^{\prime}}\left|\nabla_{x} \varphi\left(h^{\varepsilon}(s), s\right)\right|^{2} d s_{x}=O\left(\varepsilon^{2}\right) .
$$

The assumption (4.56) and estimate (4.57) prove that

$$
\int_{\Gamma^{\prime}}\left|\partial_{s} \nabla_{x} \varphi(x)\right|^{2} d s_{x}=o(1), \quad \varepsilon \rightarrow 0 .
$$

Also, from (4.54) it follows that

$$
\int_{\Gamma^{\varepsilon} \backslash \Gamma_{\varepsilon}^{\prime}}\left|\nabla_{x}^{2} \varphi(x)\right|^{2} d s_{x} \leq c \max _{q=1, \ldots, Q} \varepsilon^{2 \Lambda_{q}-3} .
$$

The second inequality in (4.58) shows that $\nabla_{x} \varphi \in H^{1 / 2}\left(\Gamma^{\varepsilon}\right)$ and $\left\|\nabla_{x} \varphi ; H^{1 / 2}\left(\Gamma^{\varepsilon}\right)\right\|^{2}=$ $o(\varepsilon)$. Consequently, $\left\|\nabla_{x}^{2} \Phi_{\varphi}^{\varepsilon} ; L_{2}\left(\Omega^{\varepsilon}\right)\right\|^{2}=o(\varepsilon)$. Recalling system (4.55), we obtain relation (1.24) in which $\theta_{p}, \ldots, \theta_{p+\varkappa_{p}-1}$ are the eigenvalues of the algebraic problem (1.26). With the help of the above estimates for $\varphi$, direct calculations yield $\theta_{j}=O(\varepsilon)$. If the opening of one of the angles (e.g., $\alpha_{q}$ ) is greater than $\pi$, then we can find an eigenfunction such that the modulus $\left|\nabla_{x}^{2} \varphi\right|$ of the second gradient is estimated from below by $\mathrm{cr}_{q}^{\lambda-1}$, where $c>0$ and $\lambda \in(3 / 2,2)$. The Hadamard formula predicts the inequality $\theta_{j} \geq c \varepsilon^{2 \lambda-3}$; therefore, the spectral problem (1.26) is an appropriate substitute of problem (1.26) in the case of nonconvex piecewise smooth domains.

\section{$\S 5$. Discussion}

1. Comparison of two approaches. The results of [7] also make it possible to construct the asymptotic expansions of eigenvalues (this was already explained in Subsection 7 of $\S 4$ ). Now we trace how the formulas obtained by one of the methods can be transformed into those obtained by the other method. We only consider the case where $\gamma=1$ assuming for brevity that $\lambda_{p}$ is a simple eigenvalue and $H<0$, i.e., $\Omega^{\varepsilon} \subset \Omega$ (see formula (1.1)). Then $\Psi_{\varphi}^{\varepsilon}=0$, because the right-hand side in (1.22) vanishes. Problem (1.21) takes the form

$$
\Delta_{x}^{2} \Phi_{\varphi}^{\varepsilon}(x)=0, \quad x \in \Omega^{\varepsilon}, \quad \Phi_{\varphi}^{\varepsilon}(x)=-\varphi(x), \quad \partial_{n^{\varepsilon}} \Phi_{\varphi}^{\varepsilon}(x)=-\partial_{n^{\varepsilon}} \varphi(x), \quad x \in \Gamma^{\varepsilon} .
$$

The asymptotic approximation of its solution is constructed in a standard way (see, e.g., [11, 12]) and looks like this:

$$
\Phi_{\varphi}^{\varepsilon}(x)=\varepsilon \varphi^{\prime}(x)+\chi(x) \varepsilon^{2} \partial_{n}^{2} \varphi(0, s) \widetilde{W}_{0}\left(\varepsilon^{-1}, \varepsilon^{-1}, s\right)+\widetilde{\Phi}_{\varphi}^{\varepsilon}(x),
$$

where $\widetilde{\Phi}_{\varphi}^{\varepsilon}$ is a small remainder term, $\widetilde{W}_{0}$ is the exponentially decaying function (3.23) found by the solution of the homogeneous problem (2.21)-(2.23), and $\varphi^{\prime}$ is the solution of the problem

$$
\Delta_{x}^{2} \varphi^{\prime}(x)=0, \quad x \in \Omega, \quad \varphi^{\prime}(x)=0, \quad \partial_{n} \varphi^{\prime}(x)=P(s) \partial_{n}^{2} \varphi(0, s), \quad s=x \in \Gamma^{\varepsilon} .
$$

Now, putting $\varphi=u_{p}$ in (1.20), we obtain the algebraic equation

$$
-\lambda_{p}^{-1}\left(\left(\Delta_{x} \Phi_{u_{p}}^{\varepsilon}, \Delta_{x} \Phi_{u_{p}}^{\varepsilon}\right)_{\Omega^{\varepsilon}}+\left(\Delta_{x} u_{p}, \Delta_{x} u_{p}\right)_{\Omega_{\backslash \Omega^{\varepsilon}}}\right)=\vartheta_{p}\left(\Delta_{x} u_{p}, \Delta_{x} u_{p}\right)_{\Omega^{\varepsilon}} .
$$

We find the asymptotic approximations of the terms in this formula. In the last scalar product, integration can be extended to the domain $\Omega$ with an error of $O(\varepsilon)$; hence, by 
(1.11) and (2.4), this scalar product is $\lambda_{p}+O(\varepsilon)$. In the second summand in parentheses on the left, integration is over a narrow boundary strip:

$$
\begin{aligned}
\left(\Delta_{x} u_{p}, \Delta_{x} u_{p}\right)_{\Omega \backslash \Omega^{\varepsilon}} & =\int_{\Gamma} \int_{\varepsilon H\left(\varepsilon^{-1} s, s\right)}^{0}\left|\Delta_{x} u_{p}(x)\right|^{2}(1+n \varkappa(s)) d n d s \\
& =-\varepsilon \int_{\Gamma} \bar{H}(s)\left|\partial_{n}^{2} u_{p}(0, s)\right|^{2} d s+O\left(\varepsilon^{2}\right) .
\end{aligned}
$$

To compute the first scalar product, we use the asymptotic representation (5.1). Observe that the smooth term $\varepsilon \varphi^{\prime}$ contributes $O\left(\varepsilon^{3 / 2}\right)$ and can be neglected. The boundary layer contribution is of order of $\varepsilon^{4} \varepsilon^{-4} \varepsilon$, where the first factor is the squared coefficient $\varepsilon^{2}$ of the cutoff function $\chi$ on the right-hand side of (5.1), the second factor comes as a result of two-fold differentiation with respect to the fast variables, and the third results from integration over the variable $n$, because $O(\exp (-\delta|n| / \varepsilon))$ decays exponentially with the rate $\widetilde{W}_{0}$. The scalar product itself needs averaging, which can be done, as in (5.2), with the help of (2.17). As a result, we obtain

$$
\left(\Delta_{x} \Phi_{u_{p}}^{\varepsilon}, \Delta_{x} \Phi_{u_{p}}^{\varepsilon}\right)_{\Omega^{\varepsilon}}=\varepsilon \int_{\Gamma}\left|\partial_{n}^{2} u_{p}(0, s)\right|^{2} \int_{\Pi(s)}\left|\Delta_{\xi} \widetilde{W}_{0}(\xi, s)\right|^{2} d \xi d s+O\left(\varepsilon^{3 / 2}\right) .
$$

Thus,

$$
\lambda_{p}^{2} \vartheta_{p}=\varepsilon \int_{\Gamma}\left|\partial_{n}^{2} u_{p}(0, s)\right|^{2}\left\{-\bar{H}(s)+\int_{\Pi(s)}\left|\Delta_{\xi} \widetilde{W}_{0}(\xi, s)\right|^{2} d \xi\right\} d s+O\left(\varepsilon^{3 / 2}\right) .
$$

Formulas (2.27) and (2.28) show that the expression in braces coincides with the coefficient $-P(s)=-P_{00}(s)$ in the expansion (2.25); i.e., in accordance with the calculations in Subsection 2 of $\S 2$, we have $\lambda_{p}^{2} \vartheta_{p}=-\varepsilon \lambda_{p}^{\prime}$. Substituting this in (1.19), we arrive at the same formula as in $\S 2$ :

$$
\frac{1}{\lambda_{p}^{\varepsilon}}=\frac{1}{\lambda_{p}}-\varepsilon \frac{\lambda_{p}^{\prime}}{\lambda_{p}^{2}}+O\left(\varepsilon^{1+\delta}\right) \quad \Rightarrow \quad \lambda_{p}^{\varepsilon}=\lambda_{p}+\varepsilon \lambda_{p}^{\prime}+O\left(\varepsilon^{1+\delta}\right) .
$$

2. Other types of boundary perturbations. If $\gamma=1$, the above arguments and calculations make it possible to study also other perturbations of the boundary. In Figure 8, we depicted two locally periodic perturbations: small holes of diameter $O(\varepsilon)$ located at a distance of $O(\varepsilon)$ from the boundary and from one another, and a periodic family of boundary cracks. In both cases, the boundary $\Gamma^{\varepsilon}$ cannot be described as in (1.1), but all our arguments remain valid. Moreover, the domain on the right in Figure 8 fails to be Lipschitzian, and the domain on the left has smooth boundary, but the Lipschitz constant grows unboundedly as $\varepsilon \rightarrow+0$.

If $\gamma<1$, then the boundary layer is localized near the curve $\Gamma_{0}^{\varepsilon}=\{x \in \mathcal{V}: s \in \Gamma$, $\left.n=\varepsilon^{\gamma} H_{0}(s)\right\}$, and the form of $\Gamma^{\varepsilon}$ outside the $c \varepsilon$-neighborhood of the contour $\Gamma_{0}^{\varepsilon}$ is to a large extent irrelevant. Figure 9 illustrates two types of perturbation, and in the second of them we have periodicity only in a small neighborhood of the contour $\Gamma_{0}^{\varepsilon}$. In both cases, the eigenvalues of problem (1.3), (1.4) in the singularly perturbed domain have the asymptotic properties described in Theorem 3.1 in the case where $\gamma<1, H_{0} \neq \equiv$.

Remark 5.1. What was said in the preceding paragraph also remains valid for $\gamma=0$; this case is not touched upon in the present paper.

3. On the concept of a smooth image of singularly perturbed domains. In the paper [12] it was observed that for various perturbations of spectral boundary-value problems it is possible to find a regular perturbation

$$
\Gamma(\varepsilon)=\left\{x \in \mathcal{V}: s \in \Gamma, n=\varepsilon^{\gamma} h(s)\right\}, \quad h \in C^{\infty}(\Gamma),
$$



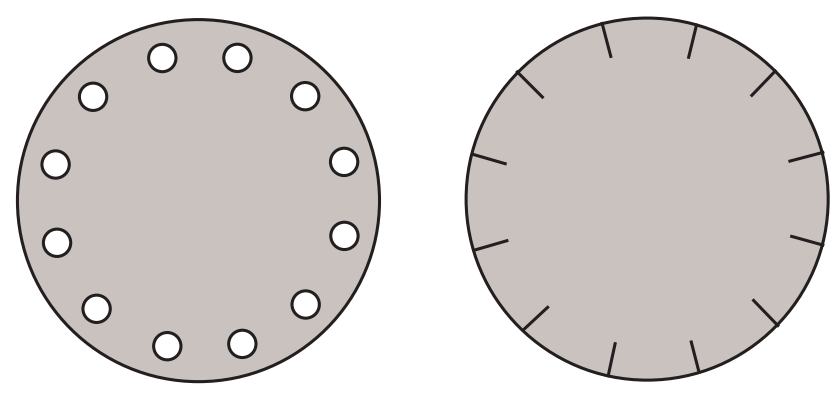

FIGURE 8

of the boundary $\Gamma$ such that the spectral problem in the domain $\Omega(\varepsilon)$ bounded by the contour (5.3) provides a two-term (higher accuracy) asymptotic expansion for eigenvalues and eigenfunctions of singularly perturbed problems. Such an interpretation of the results of the asymptotic analysis is possible for boundary-value problems with a small parameter at higher derivatives (in equations and in boundary conditions) in thin domains (for example, in the case of a strip $\Omega \times(-\varepsilon / 2, \varepsilon / 2) \subset \mathbb{R}^{3}$ with small thickness $\varepsilon$ ) and in a domain with a rapidly oscillating boundary (the case of $\gamma=1$ in the definition (1.1)).

The concept of the smooth image of singularly perturbed domains turned out to be useful for the modeling of thin elastic plates (see [57, 58, [59]) and rough surfaces of deformable bodies (see [20, 21])). The "wall-laws", which were invented only after the paper [12] (see 13, 14] and others), also fit in with this concept.

We explain how to use the asymptotic formulas obtained in $\S \S 2$ and 3 to form the smooth image of the rapidly oscillating boundary (1.1) in the framework of the Kirchhoff plates theory. Note that, in accordance with Subsection 1 of $\S 2$, the eigenvalue asymptotic approximation for problem (1.3), (1.4) in the domain $\Omega^{\varepsilon}$ bounded by a regularly perturbed contour (5.3) has the form (2.1), where the $\lambda_{q}^{\prime}$ are the eigenvalues of the matrix $\mathbf{T}^{p}$ with the entries (2.15).

If $\gamma>1$ and the periodic perturbation (1.1) of the boundary $\Gamma$ is slanting, then, by Theorem 3.1, the first two asymptotic terms for the eigenvalues of problem (1.3), (1.4) in the domain $\Omega^{\varepsilon}$ coincide with those in the case where the profile function has the form (2.18) (see Subsection 1 of $\S 2$ and, in particular, formula (3.14)). In other words, the shift $h(s)$ of the boundary (5.3) is determined by the averaged function $\bar{H}$ (see (1.15) ).

If $\gamma=1$, i.e., the sizes of the hollows and (or) bumps are comparable to the period, then the role of the function $h$ that determines an asymptotically equivalent regularly perturbed boundary (5.3) is played by the coefficient in the expansion (2.25) of the special solution $W_{0}$ of the homogeneous problem (2.21)-(2.23) (see formula (2.32).

The same relation (2.32) remains valid in the case where $\gamma \in(0,1)$ under the additional condition $H_{0} \equiv 0$ (see the restriction (1.16) and the definition (3.15)), but, first, in identity (5.3) for the regularly perturbed boundary $\Gamma(\varepsilon)$ we have the smaller factor $\varepsilon^{1}$, rather than $\varepsilon^{\gamma}$ as in the Hadamard formula (see the discussion in Subsection 1 of $\S 1$ ), and, second, the coefficient $P$ in the expansion (2.25) of the solution of problem (2.39) in the cut strip (2.38) is independent of the variable $s \in \Gamma$. In the situation where $H_{0} \not \equiv 0$ and $\gamma<1$, the smooth image (5.3) of the rapidly oscillating and deeply indented boundary (1.1) is given by formula (2.44), where $H_{0}(s)$ is the minimal value of the profile function $[0,1] \ni \eta \mapsto H(\eta, s)$ (see Theorem 3.1 and relation (3.15)).

Whenever $\Omega \subset \Omega(\varepsilon)$ (or $\Omega(\varepsilon) \subset \Omega$ ), the eigenvalues of problem (1.3), (1.4) satisfy $\lambda_{p} \geq \lambda_{p}(\varepsilon)$ (or $\left.\lambda_{p} \leq \lambda_{p}(\varepsilon)\right)$. As was mentioned in Remark 2.2 the same relationship is 

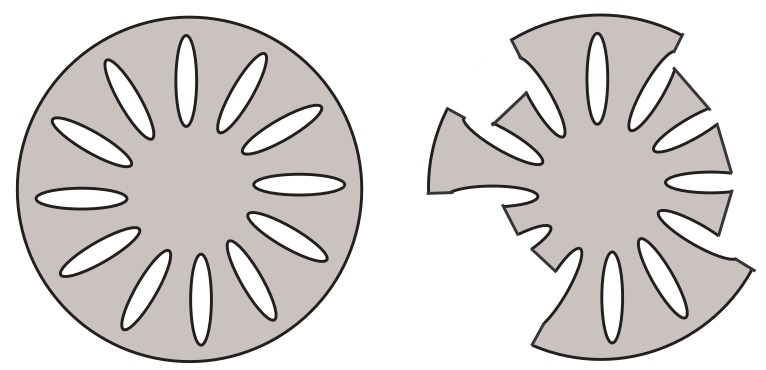

FiguRE 9

preserved also for the eigenvalue $\lambda_{p}^{\varepsilon}$ with $\varepsilon$ sufficiently small, i.e., if $\varepsilon \in\left(0, \varepsilon_{p}\right]$ with some $\varepsilon_{p}>0$.

Yet another important point deserves mention: the profile function for the regularly perturbed boundary (5.3) does not depend on the number $p$ of the eigenvalue $\lambda_{p}^{\varepsilon}$. The same function can be used for modeling other form functionals (cf. [35, 36]).

\section{REFERENCES}

[1] O. A. Ladyzhenskaya, Boundary value problems of mathematical physics, Nauka, Moscow, 1973; English transl., Appl. Math. Sci., vol. 49, Springer, New York, 1985. MR0599579 (58:29032) MR0793735 (87f:35001)

[2] M. Sh. Birman and M. Z. Solomyak, Spectral theory of selfadjoint operators in Hilbert space, Leningrad. Univ., Leningrad, 1980; English transl., D. Reidel Publ. Co., Dordrecht, 1987. MR0609148 (82k:47001) MR.1192782 (93g:47001)

[3] S. G. Mikhlin, Variational methods in mathematical physics, 2nd ed., Nauka, Moscow, 1970; English transl. of 1st ed., Pergamon Press Book, New York, 1964. MR 0353111 (50:5597) MR0172493 $(30: 2712)$

[4] S. A. Nazarov, Asymptotic analysis of thin plates and rods. Vol. 1. Dimension reducing and integral estimates, Nauchn. Kniga, Novosibirsk, 2002. (Russian)

[5] J. Hadamard, Mémoire sur le problème d'analyse relatif à l'équilibre des plaques élastiques encastrées, Euvres. de Jacques Hadamard. T. 2, Centre Nat. Rech. Sci., Paris, 1968, pp. 515-631. MR0230598(37:6158)

[6] U. Mosco, Convergence of convex sets and of solutions of variational inequalities, Adv. Math. 3 (1969), 510-585. MR0298508 (45:7560)

[7] V. Kozlov, On the Hadamard formula for nonsmooth domains, J. Differential Equations 230 (2006), no. 2, 532-555. MR2269932(2007h:35244)

[8] $\quad L^{q}$-perturbations of leading coefficients of elliptic operators: asymptotics of eigenvalues, Abstr. Appl. Anal. 2006, Art. ID 26845, 15 pp. MR2251795 (2007d:35051)

[9] V. G. Maz'ya, S. A. Nazarov, and B. A. Plamenevskiř, Asymptotic behavior of solutions of elliptic boundary value problems under singular perturbations of the domain, Tbilis. Gos. Univ., Tbilisi, 1981; English transl., Asymptotic theory of elliptic boundary value problems in singularly perturbed domains. Vol. I, Oper. Theory Adv. Appl., vol. 111, Birkhäuser, Basel, 2000. MR0626525 (83b:35022) MR1779977 (2001e:35044a)

[10] A. M. Il'in, Matching of asymptotic expansions of solutions of boundary value problems, Nauka, Moscow, 1989; English transl., Transl. Math. Monogr., vol. 102, Amer. Math. Soc., Providence, RI, 1992. MR 1007834 (90i:35062); MR1182791 (93g:35016)

[11] E. Sánchez-Palencia, Nonhomogeneous media and vibration theory, Lecture Notes in Phys., vol. 127, Springer-Verlag, Berlin-New York, 1980, 398 pp. MR:0578345 (82j:35010)

[12] S. A. Nazarov, Binomial asymptotic behavior of solutions of spectral problems with singular perturbations, Mat. Sb. 181 (1990), no. 3, 291-320; English transl., Math. USSR-Sb. 69 (1991), no. 2, 307-340. MR 1049991 (91d:35160)

[13] Y. Achdou and O. Pironneau, Domain decomposition and wall laws, C. R. Acad. Sci. Paris. Sér. I 320 (1995), 541-547. MR.1322334 (95k:76087) 
[14] B. Mohammadi, O. Pironneau, and F. Valentin, Rough boundaries and wall laws, Internat. J. Numer. Methods Fluids 27 (1998), no. 1-4, Special Issue, 169-177. MR 1602088

[15] Y. Amirat, G. A. Chechkin, and R. R. Gadyl'shin, Asymptotics of simple eigenvalues and eigenfunctions for the Laplace operator in a domain with oscillating boundary, Zh. Vychisl. Mat. Mat. Fiz. 46 (2006), no. 1, 102-115; English transl., Comput. Math. Math. Phys. 46 (2006), no. 1, 97-110. MR2239730 (2007d:35009)

[16] W. Jäger and A. Mikelić, On the roughness-induced effective boundary conditions for an incompressible viscous flow, J. Differential Equations 170 (2001), 96-122. MR1813101 (2002b:76049)

[17] N. Neuss, M. Neuss-Radu, and A. Mikelić, Effective laws for the Poisson equation on domains with curved oscillating boundaries, Appl. Anal. 85 (2006), no. 5, 479-502. MR2213071 (2006k:35012)

[18] D. Bresch and D. Gérard-Varet, Roughness-induced effects on the quasi-geostrophic model, Comm. Math. Phys. 253 (2005), no. 1, 81-119. MR2105638 (2005i:76115)

[19] E. Sánchez-Palencia and P. Suquet, Friction and homogenization of a boundary, Free Boundary Problems: Theory and Applications, Vols. I, II (Montecatini, 1981) (A. Fasano, M. Primicerio, eds.), Res. Notes in Math., No. 78, Pitman, Boston, MA, 1983, pp. 561-571. MR0714936 (86b:73036)

[20] S. A. Nazarov, Asymptotic behavior of solutions and the modeling of problems in the theory of elasticity in a domain with a rapidly oscillating boundary, Izv. Ross. Akad. Nauk Ser. Mat. 72 (2008), no. 3, 103-158; English transl., Izv. Math. 72 (2008), no. 3, 509-564. MR2432755 (2009i:35317)

[21] _ Eigenoscillations of an elastic body with a rough surface, Prikl. Mekh. Tekhn. Fiz. 48 (2007), no. 6, 103-114; English transl., J. Appl. Mech. Tech. Phys. 48 (2007), no. 6, 861-870. MR:2373776 (2008g:74056)

[22] T. A. Mel'nyk and S. A. Nazarov, Asymptotic structure of the spectrum of the Neumann problem in a thin comb-like domain, C. R. Acad. Sci. Paris Sér. I 319 (1994), no. 12, 1343-1348. MR1310684 (95i:35206)

[23] Asymptotic behavior of the solutions of the Neumann spectral problem in a domain of "tooth comb" type, Tr. Semin. im. I. G. Petrovskogo No. 19 (1996), 138-173; English transl., J. Math. Sci. (N. Y.) 85 (1997), no. 6, 2326-2346. MR1693707(2001a:35136)

[24] D. Blanchard, A. Gaudiello, and T. A. Mel'nyk, Boundary homogenization and reduction of dimension in a Kirchhoff-Love plate, SIAM J. Math. Anal. 39 (2008), no. 6, 1764-1787. MR2390313 (2009b:35008)

[25] A. Gaudiello and E. Zappale, Junction in a thin multidomain for a fourth order problem, Math. Models Methods Appl. Sci. 16 (2006), no. 12, 1887-1918. MR2287334 (2008i:74078)

[26] S. A. Nazarov, Asymptotic expansion of the solution of the Dirichlet problem for an equation with rapidly oscillating coefficients in a rectangle, Mat. Sb. 182 (1991), no. 5, 692-722; English transl., Math. USSR-Sb. 73 (1992), no. 1, 79-110. MR1124104 (92h:35038)

[27] S. A. Nazarov and M. V. Olyushin, Perturbations of eigenvalues of the Neumann problem due to variations of a domain's boundary, Algebra i Analiz 5 (1993), no. 2, 169-188; English transl., St. Petersburg Math. J. 5 (1994), no. 2, 371-387. MR1223177 (94f:35020)

[28] F. Blanc and S. A. Nazarov, Asymptotics of solutions to the Poisson problem in a perforated domain with corners, J. Math. Pures Appl. (9) 76 (1997), no. 10, 893-911. MR1489944 (98h:35017)

[29] G. Caloz, M. Costabel, M. Dauge, and G. Vial, Asymptotic expansion of the solution of an interface problem in a polygonal domain with thin layer, Asymptot. Anal. 50 (2006), no. 1-2, 121-173. MR 2286939(2009d:35048)

[30] S. A. Nazarov, Dirichlet problem in an angular domain with rapidly oscillating boundary: Modeling of the problem and asymptotics of the solution, Algebra i Analiz 19 (2007), no. 2, 183-225; English transl., St. Petersburg Math. J. 19 (2008), no. 2, 297-326. MR2333903 (2008h:35038)

[31] _ Asymptotic behavior of the solution of the Dirichlet problem in an angular domain with a periodically changing boundary, Mat. Zametki 49 (1991), no. 5, 86-96; English transl., Math. Notes 49 (1991), no. 5-6, 502-509. MR.1137177 (92g:35062)

[32] $\ldots$, The Neumann problem in angular domains with periodic and parabolic perturbations of the boundary, Trudy Moskov. Mat. Obshch. 69 (2008), 182-241; English transl., Trans. Moscow Math. Soc. 2008, 153-208. MR2549447|(2010h:35099)

[33] D. Bucur and G. Buttazzo, Variational methods in shape optimization problems, Progr. Nonlinear Differential Equations Appl., vol. 65, Birkhäuser Boston, Inc., Boston, MA, 2005. MR2150214 (2006j:49002)

[34] A. Henrot, Extremum problems for eigenvalues of elliptic operators, Frontiers in Math., Birkhäuser Verlag, Basel, 2006. MR2251558 (2007h:35242)

[35] J. Sokolowski and L.-P. Zolésio, Introduction to shape optimization. Shape sensitivity analysis, Springer Ser. Comput. Math., vol. 16, Springer-Verlag, Berlin, 1992. MR.1215733 (94d:49002) 
[36] M. C. Delfour and J.-P. Zolésio, Shapes and geometries. Analysis, differential calculus, and optimization, Adv. in Design and Control, vol. 4, SIAM, Philadelphia, PA, 2001. MR1855817 (2002i:49002)

[37] B. Kawohl, Some nonconvex shape optimization problems, Optimal Shape Design (Tróia, 1998), Lecture Notes in Math., vol. 1740, Springer, Berlin, 2000, pp. 7-46. MR1804684

[38] N. S. Nadirashvili, Rayleigh's conjecture on the principal frequency of the clamped plate, Arch. Rational Mech. Anal. 129 (1995), no. 1, 1-10. MR.1328469 (97j:35113)

[39] M. S. Ashbaugh and R. D. Benguria, On Rayleigh's conjecture for the clamped plate and its generalization to three dimensions, Duke Math. J. 78 (1995), no. 1, 1-17. MR.1328749 (97j:35111)

[40] M. Sh. Birman, Variational methods of solution of boundary problems analogous to the method of Trefftz, Vestnik Leningrad. Univ. 11 (1956), vyp. 3, 69-89. (Russian) MR0080867 (18:316f)

[41] I. Babushka, Stabilität des Definitionsgebietes mit Rücksicht auf grundlegende Probleme der Theorie der partiellen Differentialgleichungen auch im Zusammenhang mit der Elastizitätstheorie. I, II, Czechoslovak Math. J. 11 (1961), 76-105; 165-203. (Russian) MR0125326 (23:A2629)

[42] V. G. Maz'ya and S. A. Nazarov, Paradoxes of the passage to the limit in solutions of boundary value problems for the approximation of smooth domains by polygons, Izv. Akad. Nauk SSSR Ser. Mat. 50 (1986), no. 6, 1156-1177; English transl., Math. USSR-Izv. 29 (1987), no. 3, 511-533. MR $0883157(88 \mathrm{i}: 35016)$

[43] I. Babushka and J. Pitkäranta, The plate paradox for hard and soft simple support, SIAM J. Math. Anal. 21 (1990), no. 3, 551-576. MR:1046789 (91b:73059)

[44] S. A. Nazarov, Polynomial property of selfadjoint elliptic boundary value problems, and the algebraic description of their attributes, Uspekhi Mat. Nauk 54 (1999), no. 5, 77-142; English transl., Russian Math. Surveys 54 (1999), no. 5, 947-1014. MR1741662(2001k:35073)

[45] S. Agmon and L. Nirenberg, Properties of solutions of ordinary differential equations in Banach space, Comm. Pure Appl. Math. 16 (1963), 121-239. MR0155203 (27:5142)

[46] V. A. Kondrat'ev, Boundary value problems for elliptic equations in domains with conical or angular points, Trudy Moskov. Mat. Obshch. 16 (1967), 209-291; English transl. in Trans. Moscow Math. Soc. 1967 (1968). MR 0226187 (37:1777)

[47] V. G. Maz'ya and B. A. Plamenevskiur, The coefficients in the asymptotics of solutions of elliptic boundary value problems with conical points, Math. Nachr. 76 (1977), 29-60. (Russian) MR0601608 $(58: 29176)$

[48] Estimates in $L_{p}$ and in Hölder classes, and the Miranda-Agmon maximum principle for the solutions of elliptic boundary value problems in domains with singular points on the boundary, Math. Nachr. 81 (1978), 25-82. (Russian) MR0492821 (58:11886)

[49] S. A. Nazarov and B. A. Plamenevski1̆, Elliptic problems in domains with piecewise smooth boundaries, Nauka, Moscow, 1991; English transl., de Gruyter Expos. in Math., vol. 13, Walter de Gruyter, Berlin, 1994. MR.1283387 (95h:35001)

[50] V. A. Kozlov, V. G. Maz'ya, and J. Rossmann, Elliptic boundary value problems in domains with point singularities, Math. Surveys Monogr., vol. 52, Amer. Math. Soc., Providence, RI, 1997. MR:1469972 (98f:35038)

[51] M. L. Williams, Stress singularities resulting from various boundary conditions in angular corners of plate, J. Appl. Mech. 4 (1952), no. 4.

[52] M. I. Vishik and L. A. Lyusternik, Regular degeneration and boundary layer for linear differential equations with small parameter, Uspekhi Mat. Nauk 12 (1957), no. 5, 3-122. (Russian) MR0096041 (20:2539)

[53] S. A. Nazarov and Yu. A. Romashev, Variation of the intensity coefficient under destruction of a dam between collinear splits, Izv. Akad. Nauk Armyan. SSR. Mekh. 35 (1982), no. 4, 30-40. (Russian)

[54] S. A. Nazarov and A. S. Slutskiǔ, Homogenization of a mixed boundary value problem in a domain with anisotropic fractal perforation, Izv. Ross. Akad. Nauk Ser. Mat. 74 (2010), no. 2, 165-194. (Russian) MR2675272

[55] V. Adolfsson and J. Pipher, The inhomogeneous Dirichlet problem for $\Delta^{2}$ in Lipschitz domains, J. Funct. Anal. 159 (1998), no. 1, 137-190. MR1654182 (99m:35048)

[56] V. A. Kozlov, V. G. Maz'ya, and J. Rossmann, Spectral problems associated with corner singularities of solutions to elliptic equations, Math. Surveys Monogr., vol. 85, Amer. Math. Soc., Providence, RI, 2001. MR.1788991 (2001i:35069)

[57] I. S. Zorin and S. A. Nazarov, The boundary effect in the bending of a thin three-dimensional plate, Prikl. Mat. Mekh. 53 (1989), no. 4, 642-650; English transl., J. Appl. Math. Mech. 53 (1989), no. 4, 500-507. MR:1022416 (90m:73045) 
THE SPECTRUM ASYMPTOTICS

[58] S. A. Nazarov, On the three-dimensionality effect near the tip of a crack in a thin plate, Prikl. Mat. Mekh. 55 (1991), no. 3, 500-510; English transl., J. Appl. Math. Mech. 55 (1991), no. 3, 407-415 (1992). MR1134619 (92k:73061)

[59] _ Boundary layers and boundary conditions for hinge support for thin plates, Zap. Nauchn. Sem. S.-Peterburg. Otdel. Mat. Inst. Steklov. (POMI) 257 (1999), 228-287; English transl., J. Math. Sci. 108 (2002), no. 5, 806-850. MR.1754703(2001c:74026)

Department of Mathematics, Linkoping University, 58183 Linkoping, Sweden

E-mail address: vlkoz@mai.liu.se

Institute of Mechanical Engineering Problems, Russian Academy of Sciences, Bol'shoŭ Pr. V.O. 61, St. Petersburg 199178, Russia

E-mail address: srgnazarov@yahoo.co.uk

Received 15/JUN/2010

Translated by A. PLOTKIN 